DEB9 008386

\title{
Irreversible Adsorption of Phenolic Compounds by Activated Carbons
}

\author{
Terry M. Grant* and C. Judson King \\ *Ph.D. Thesis \\ Applied Science Division \\ Lawrence Berkeley Laboratory \\ 1 Cyclotron Road \\ Berkeley, California 94720
}

December 1988

\section{DISCLAIMER}

This report was prepared as an sccount of work sponsored by an agency of the United States Government. Neither the United States Government nor any agency thereof, nor any of their employes, makes any warranty, express or implied, or assumes any lezal liability or responsibility for the accuracy, completeness, or usefulness of any information, apparatus, product, or process disclosed, or represents that its use would not infringe privately owned rights. Reference herein to any specific commercial product, process, or service by trade name, trademark, manufacturer, or otherwise does not necessarily constilute or imply its endorsement, recommendation, or favoring by the United States Government or any agency thereof. The views and opinions of authors expresued herein do not necessurily state or reflect those of the United States Government or any agency thereof. 


\section{Irreversible Adsorption of Phenolic Compounds \\ by Activated Carbons}

Terry Michael Grant

\section{abatraet}

Studies were undertaken to determine the reasons why phenolic sorbates can be difficult to remove and recover from activated carbons. The chemical properties of the sorbate and the adsorbent surface, and tha influences of changes in the adsorption and desorption conditions were investigated.

Comparison of isotherms established after different contact times or at different temperatures indicated that phenolic compounds react on sarbon surfaces. The reaction rate is a strong function of tomperature.

Regeneration of carbons by leaching with acetone recovered at least as much phenol as did regeneration with other solvents or with displacers, and hence was used to define irreversible adsorption operationally. 
The physiochemical properties of adsorbents influenced irreversible uptakes. Carbons oxidized with nitric acid or hydrogen peroxide were more regenerable than the parent carbons. The abundance rather the nature of surface functional groups seemed to have the greatest impact on uptakes. Carboxylic acid and ester groups on polymeric resins did not promote irreversibility, but phenolic groups did. Mineral ash promoted irreversibility, but irraversible adsorption also occurred on sorbates containing little or no ash. Electron paramagnetic resonance measurements Indicated that unpaired electrons were probably not responsible for irreversible adsorption on carbonaceous adsorbents.

Sorbates differed markedly in their tendencies to undergo irreversible adsorption. Monohydroxybenzes were more reactive than non-phenolic compounds, but less reactive than dihydroxybenzenes. There is a semiquantitative correlation between reactivities and critical oxidation potentials for the sorbates studied.

Oxidative coupling of sorbate molecules is probably responsible for irreversible adsorption. polymers that are expected products of coupling reactions were identified in a regenerant mixture. Further, conditions that promote oxidation of phenolic compounds also promote irreversibilities. High temperature, alkaline conditions, 
and readily available oxygen are examples. 
Dedication

To

Karen Lynn Deeter

with

Love and Admiration 


\section{Acknowledgenents}

I am thankful for my associations with Professor $c$. Judson king. He is not only an outstanding research advisor, but also an outstanding friend. Some of my fondest memories of graduate school involve treks through Buckskin Gulch and down the virgin River with Dr. King and his Boy scout troop. I hope that our paths cross frequently.

Professors Scott Iynn and Leo Brewer provided thoughtful comments on the final drafts of this dissertation. Professor Lynn deserves additional thanks for his guidance on my qualifying examination.

Some of the experiments reported in this dissertation were carried out by others. Thanks are due to Kristen Peterson, Randy Sadier, and Gina zhanley who labored as undergraduate researchers. Thanks are also due to Dr. Curtis Munson, Dr. Paul Skerker, and to Mr. V. Tashinian for their analyses.

Several colleagues contributed to my graduate experiences. Curt Munson, Rod Thompson, and John Senetar enticed me to enter the group and I have forgiven them. Tarric El-Sayed is an accomplished pathfinder, and I enjoyed our wilderness adventures immeasurably. Bill Rixey 
helped me to understand several technical issues and is a generous friend. The team of Dave Wallack and Bob(?) Fields has been most amusing, as have been some of Loree Poole's jokes. Paul Sanchez, Spiros Papadakis, Mark Smith, Tony Garcia, Janet Tamada, Dan Arenson, Baishen Liang and Jack Starr have also been valuable sources of ideas and entertainment. Thank you all for the camaraderie.

I am grateful to my family for their encouragement and support. I have never felt at home when away from them.

Finally, I cannot zepay the debt I owe to my fiancee Karen. Karen helped me through many stressful times and made many sacrifices on my behalf.

This work was supported by the Morgantown Energy Technology Center, Morgantown, wV, through the Assistant Secretary for Fossil Energy, Office of surface coal Gasification, Advanced Process Research Program of the US Department of Energy under Contract No. DE-ACO3-765100098 and also by Director's Funds, Lawrence Berkeley Laboratory. 
Table of content:

Abstract

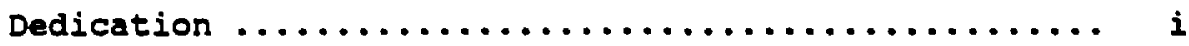

Acknowledgements $\ldots \ldots \ldots \ldots \ldots \ldots \ldots \ldots \ldots \ldots \ldots \ldots \ldots$ ii

Table of Contents $\ldots \ldots \ldots \ldots \ldots \ldots \ldots \ldots \ldots \ldots \ldots$ iv

List of Figures $\ldots \ldots \ldots \ldots \ldots \ldots \ldots \ldots \ldots \ldots \ldots \ldots \ldots$ viii

List of Tables $\ldots \ldots \ldots \ldots \ldots \ldots \ldots \ldots \ldots \ldots \ldots \ldots \ldots \ldots$ xi

Chapter I Background $\ldots \ldots \ldots \ldots \ldots \ldots \ldots \ldots \ldots \ldots \ldots \ldots \ldots$. 1

Chapter 2 Evidence for a Chenical Reaction ........ 16

2.1 Sources, Pretreatment, and Surface areas of Adsorbents ........................ 16

2.2 Determination of Adsorption Amounts and Isotherms ...................... 20

2.3 Comparison of Liquid-Vapor and Solid-Liquid Surface Excesses .................... 22

2.4 Rates of Adsorption onto Filtrasorb-100

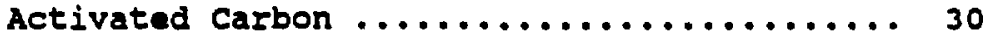

2.5 Influence of Temperature on Adsorption Amounts ........................... 35

2.6 References $\ldots \ldots \ldots \ldots \ldots \ldots \ldots \ldots \ldots \ldots \ldots \ldots, 42$

Chapter 3 Regeneration of Activated Carbona ........43

3.1 Goal: Establish a Working Definition of Irreversible Adsorption 
3.2 Alternative Methods of Regeneration $\ldots \ldots \ldots 44$

3.3 Regeneration with Liquids $\ldots \ldots \ldots \ldots \ldots \ldots, 47$

3.3.1 Effectiveness of Various Liquid Regenerants $\ldots \ldots \ldots \ldots \ldots \ldots \ldots \ldots \ldots \ldots \ldots, 48$

3.3.2 Effectiveness of Phenolic Displacers ..... 56

3.4 References $\ldots \ldots \ldots \ldots \ldots \ldots \ldots \ldots \ldots \ldots \ldots \ldots \ldots 60$

Chapter \& Characterising the Reaction ............

4.1 Influences of Adsorbent Properties on Irraversible Adsorption ..............63

4.1.1 XAD-4, a Reversibly Adsorting Resin ...... 63

4.1.2 Surface Properties of Carbon That Affect Total Uptakes of Phenolic Compounds ...... 68

4.1.3 Characterization of Activated Carbon surface chemistries ................ 71

4.1.3.1 Elemental Analysis .............. 72

4.1 .3 .2 Active Hydrogen $\ldots \ldots \ldots \ldots \ldots \ldots \ldots \ldots \ldots .75$

4.1.3.3 Fourier Transform Infrared spectroscopy . 81

4.1.4 Influences of Functional Groups on Irreversible Adsorption ............. 82

4.1.4.1 Experimental Procedure ............. 83

4.1.4.2 Vendor-Supplied Carbons ............ 84

4.1.4.3 Oxidized Carbons $\ldots \ldots \ldots \ldots \ldots \ldots \ldots \ldots . .68$

4.1.4.4 Carbons Treated by Other Methods ....... 97

4.1.4.5 Functional Groups on Polymeric Resins ... 103

4.1.5 Influence of Carbon Ash Contents on Irreversible Adsorption ............. 110 
4.1.6 Influence of Unpaired Electrons on Irreversible Adsorption ............. 117

4.2 Influences of Sorbate Properties on Irreversible Adsorption .............. 122

4.2.1 Nonreactive Sorbates ................ 123

4.2 .2 Dihydroxybenzenes $\ldots \ldots \ldots \ldots \ldots \ldots \ldots \ldots \ldots . \ldots 128$

4.2 .3 Monohydroxybenzenes ................ 129

4.2 .4 Aniline $\ldots \ldots \ldots \ldots \ldots \ldots \ldots \ldots \ldots \ldots \ldots \ldots \ldots 140$

4.2.5 A Relative Ordering of Reactivities ...... 140

4.3 Influences of Activity and Surface

Coverage on Irreversible Adsorption ....... 145

4.3.1 Variations with Solute Activity ......... 145

4.3.2 Repetitive Loading and Regeneration ...... 151

4.4 Influences of tiquid-Phase Properties on Irreversible Adsorption .............. 155

4.4 .1 Adsorption from Hoptane $\ldots \ldots \ldots \ldots \ldots \ldots \ldots 155$

4.4 .2 Effects of $\mathrm{pH}, \ldots \ldots \ldots \ldots \ldots \ldots \ldots \ldots \ldots \ldots . \ldots 163$

4.4 .3 Availability of oxygen ............... 170

4.5 Desorbable Reaction Products .......... 177

4.6 References $\ldots \ldots \ldots \ldots \ldots \ldots \ldots \ldots \ldots \ldots \ldots \ldots \ldots . . \ldots 5$

Chapter 5 oxidative Coupling as a Mochnaine for Irreversible adsorption .............. 192

5.1 Characteristics of oxidative coupling Reactions ......................... 192

5.2 Consistency of the Reaction Characteristics with Experimental Observations .......... 195

5.2.1 Desorbable Reaction Products ........... 196 
5.2 .2 Sorbate Reactivities $\ldots \ldots \ldots \ldots \ldots \ldots \ldots \ldots 198$

5.2.2.1 Nonreactive Sorbates ............. 198

5.2 .2 .2 Hydroxybenzenes and aniline $\ldots \ldots \ldots \ldots 200$

5.2.3 Liquid Phase Properties ............. 206

5.2.3.1 A Potential Role of Molecular Oxygen .... 207

5.2 .3 .2 The Influence of $\mathrm{pH} \ldots \ldots \ldots \ldots \ldots \ldots \ldots .208$

5.2 .3 .3 Heptane Compared to Water ........... 209

5.2 .4 Adsorbent Properties ................ 210

5.2 .5 summary $\ldots \ldots \ldots \ldots \ldots \ldots \ldots \ldots \ldots \ldots \ldots \ldots \ldots .214$

5.3 References ...................... 215

Chapter s sumary and process Inplications ........218

Appendix I Chronatographie Kethods ............ 223

Appendix II Thernodyanie considerations ......... 225 


\section{List of Figures}

Figure

2.1 Surface Excesses of Phenol at Interfaces with Aqueous solutions at $25^{\circ} \mathrm{C} . \ldots \ldots \ldots \ldots \ldots \ldots \ldots \ldots$

2.2a Surface Excesses of Phenol and Resorcinol at Interfaces with Aqueous Solutions. .......... 28

2.2b Surface Excesses of Phenol and Resorcinol on

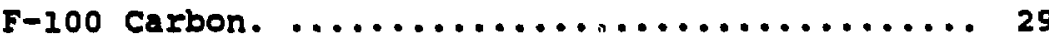

2.3 Kinetics of Adsorption for Three Sorbates on

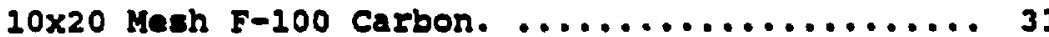

2.4 Adsorption of Phenol fron Water onto F-100 Carbon at $25^{\circ} \mathrm{C}$ for 10 Days and for 35 Days. .... 34

2.5a Adsorption of Ethanol Erom Water onto F-100 Carbon. ............................. 36

2.5b Adsorption of 5,5-Dimethylhydantoin from Water onto F-100 Carbon. ..................... 37

2.6 Adsorption of Phenol from Water onto $F-100$ Carbon at various Temperatures. ............. 38

2.7 Adsorption of Phenol from water onto $\mathrm{XAD}-4$ at $25^{\circ} \mathrm{C}$ and at $80^{\circ} \mathrm{C} . \ldots \ldots \ldots \ldots \ldots \ldots \ldots \ldots \ldots \ldots . \ldots \ldots$

2.8 Adsorption of Phenol from water onto F-100

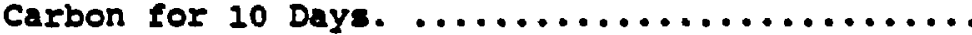

4.1 Total and Reversible Adsorption of Phenol and Resorcinol from water onto XaD 4 . ........... 66

4.2 Total and Reversible Adsorption of Phenol from Water onto XAD-4.

4.3 Reactions of Selected Functional Groups with

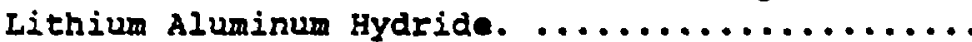

4.4 Total and Reversible Adsorption of Phenol from

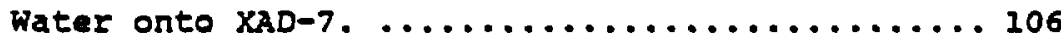

4.5 Total, Reversible and Irreversible Adsorption of Phenol from Water onto $s-761 . \ldots \ldots \ldots . . . . . .109$

4.6a Total, Reversible and Irreversible Adsorption of Nitrobenzene from Water onto Columbia Carbon. .. 126 
Figure

4.6b Total, Reversible and Irreversible Adsorption of Methoxybenzene from water onto Columbia Carbon. 127

4.7 , Total, Reversible and Irreversible Adsorption of Phenol from Water onto Columbia Carbon. ...... 130

4.8 Total, Reversible and Irreversible Adsorption of 2,4-Dimethylphenol from water onto Columbia carbon. ............................... 132

4.9 Total, Reversible and Irreversible Adsorption of p-Chlorophenol from Water onto Columbia Carbon. 134

4.10. Total, Reversible and Irreversible Adsorption of p-Methoxyphenol Iron water onto Columbia Carbon. 135

4.11 Total, Reversible and Irreversible Adsorption of p-kydroxybenzaldehyde Erom water onto Columbia

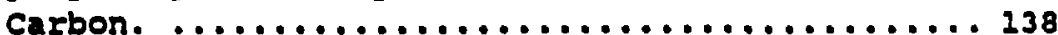

4.12 Total, Reversible and Irreversible Adsorption of p-Nitrophenol from Hater onto Columbia Carbon. . 139

4.13 Total, Reversible and Irreversible Adsorption of Aniline from water onto Columbia Carbon. ...... 141

4.14 Total, Reversible and Irreversible Adsorption of Phenol from Water onto Columbia Carbon. ....... 147

4.1.5 Total, Reversible and Irreversible Adsorption of Hydroquinone from water onto Columbia Carbon. . . 150

4.1.6a Total Uptakes of Phenol from Heptane onto Columbia Carbon. ...................... 157

4.16b Reversible Uptakes of Phenol from Heptane onto Columbia Carbon. ....................... 158

4.16C Irreversible Uptakes of Phenol Erom Heptane onto Columbia carbon. ........................ 159

4.17a Total Uptakes of Phenol from Water and from Heptane onto columbia carbon. .............. 160

4.17b Reversible uptakes of Phenol from Water and from Heptane onto columbia carbon. ............ 161

4.17C Irreversible Uptakes of Phenol from water and from Heptane onto Columbia Carbon. ........... 162

4.18a Total Uptakes of Phenol from Aqueous Solutions

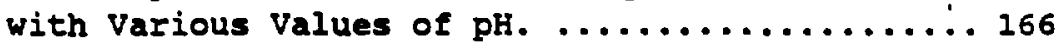


4.18b Reversible Uptakes of Phenol from Aqueous Solutions with various values of pH. ........ 167

4.18c Irreversible Uptakes of Phenol from Aqueous Solutions of various values of pH. .......... 168

4.19a Total Uptakes of Phenol from Aqueous Solutions with Various Amounts of $\mathrm{O}_{2}$ Available in the

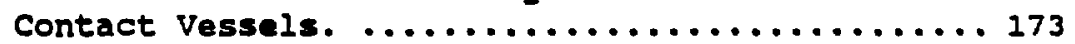

4.19b Reversible Uptakes of Phenol from Aqueous Solutions with Various amounts of $\mathrm{O}_{2}$ Available in the Contact vessels. ................. 174

4.19c Irreversible Uptakes of Phenol from Aqueous Solutions with various amounts of $\mathrm{O}_{2}$ Available In the contact vessels. ................ 175

4.20 a Total uptakes of Phenol from Aqueous Solutions with Various amounts of $\mathrm{O}_{2}$ available in the Contact vessels. .......................

$4.20 \mathrm{~b}$ Reversible Uptakes of Phenol from Aqueous Solutions with Various amounts of $\mathrm{O}_{2}$ available in the Contact vessels. ................. 179

4.20c Irreversible Uptakes of Phenol from Aqueous Solutions with Various Amounts of $\mathrm{O}_{2}$ Available in the Contact vessels. ................. 180

4.21a Mass Chromatograms for a Regenerant Mixture. ... 183

4.21b Mass Chromatograms for a Solution of Phenol in Acetone. .......................... 184

5.1 Oxidative Coupling of Phenolic Molecules. ..... 194

5.22 Estimated Amount of Irreversible Adsorption onto Columbia Carbon at solute Activity $=0.002$ (Mole Fraction) vs. Critical oxidatition Potential for Phenolic compounds. ..................... 204

5.2b Estimated Amount of Irreversible Adsorption onto Columbia Carbon at Total Adsorption = $3.0 \mu \mathrm{mole} / \mathrm{m} 2 \mathrm{vs}$. Critical Oxidatition Potential for Phenolic Compounds and Aniline. ........ 205

II-1 Activity Coefficients for Phenol in Water at

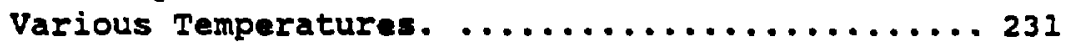


Iiat of Tables

Table

page

1.1 EPA Priority Pollutants ................. 2

1.2 Classification of Organic Adsorbates by Suzuki et

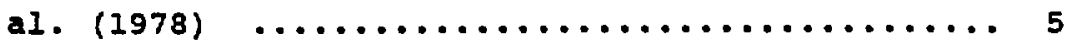

1.3 Desorption Irreversibility in the study of Yonge

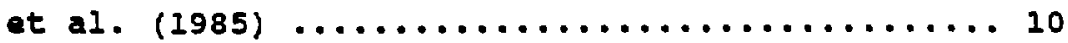

$2.1 \mathrm{a}$ Vendor-Supplied Activated Carbons Used in This study $\ldots \ldots \ldots \ldots \ldots \ldots \ldots \ldots \ldots \ldots \ldots \ldots \ldots \ldots \ldots$

2.1b. Polymeric and Pyrolyzed-Polymer Adsorbents Used in this study $\ldots \ldots \ldots \ldots \ldots \ldots \ldots \ldots \ldots \ldots \ldots \ldots$

3.1a. Batch Regeneration of Phenol from F-100 Activated Carbon .............................. 49

3.1v, Completeness of Batch Regeneration of Phenol From

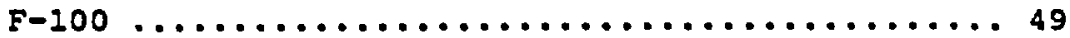

3.2 Donor-Acceptor Interaction Parameters from the Model of Drago ........................ 54

3.3 Desorption of Phenol from Witco Columbia Activated Carbon into Solvents and with Displacers .......................... 58

4.1 Elemental Analysis of Vendor-Supplied Activated Carbons .............................. 74

4.2 Active Hydrogen Contents of Vendo:-Supplied Activated Carbons ...................... 80

4.3 Comparative Regenerability of Vendor-supplied Activated Carbons ...................... 86

4.4 Weight Changes and Elemental Analyses for Oxidized Columbia ...................... 91

4.5 Surface Areas and Oxygen/Hydrogen Ratios For oxidized Columbia ..................... 91

4.6 Comparative Regenerability of Columbia and Oxidized Columbia .................... 93

4.7 Elemental Analyses for Columbia Treated in various ways $\ldots \ldots \ldots \ldots \ldots \ldots \ldots \ldots \ldots \ldots \ldots \ldots . \ldots 9$ 
4.8 Surface Areas for Columbia Treated in Various Ways ............................... 100

4.9 Comparative Regenerability of Columbia Treated in various ways ....................... ¿...

4.10 Removal of Phenol from IRC-50 ............. 108

4.11 Elemental Analysis of Columbia Treated with

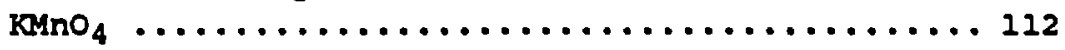

4.12 Surface Areas for Columbia Treated with $\mathrm{KMnO}_{4}$.. 114

4.13 Comparative Regenerability of Columbia Treated with $\mathrm{KMnO}_{4}$ in Various Ways ................ 114

4.14 Ash composition of Witco Columbia ........... 114

4.15 Elemental Analysis of $x \mathrm{x}-340$ and $x \mathrm{x}-348 \ldots \ldots 16$

4.16 Comparative Regenerability of XE-340 and XE-348 116

4.17 Unpaired Electrons and Comparative

Regenerability of XAD-4, XE-340, and XE-348 ... 121

4.18 Removal of 5,5-Dimethylhydantoin From F-100 with Methanol ........................ 124

4.19 Properties of substituted Benzenes ........... 144

4.20 Repetitive Adsorption and Regeneration Trials .. 153

4.21 Aqueous Buffer Solutions Used in Adsorption Experiments .......................... 165

5.1 Critical oxidation potentials of phenolic Compounds and Aniline .................. 201

II-1 Infinite Dilution Activity Coefficients for Aromatic solutes ....................... 229 
Chapter 1 Background

Separation of phenolic compounds from water receives widespread attention in the United States. A key reason for this attention is that the Environmental protection Agency designates phenol and several substituted phenols as priority pollutants. Table 1.1 shows the phenolic compounds with that designation. These compounds must be reduced to extremely low levels in public water supplies.

A second reason for the attention is that many industrial processes contaminate water with phenolic compounds. Phenols are relatively stable oxidation and pyrolysis products and are formed in processing of hydrocarbon feedstocks. In particular, coal-gasification, coal coking and petroleum refining operations emit phenols. Processes that require phenolic compounds as raw materials tend to transfer some of those compounds into effluent wacers. The proăuction of phenoilc resins is an example. Processes used to synthesize phenolics, such as the manufacture of agricultural chemicals, and those processes used to recover phenolics are also culprits.

Activated carbon is often used to treat effluents from these processes because this adsorbent has a very high capacity for phenolic compounds and can remove them almost 
Table 1.1 EPA Priority Pollutants*

Phenol

2,4-Dimethylphenol

Chlorinated Phenols

Chlorinated Cresols

Nitrophenols

Nitrocresuls

Clean Water Act Section 307 in Environmental Statutes,

Rockville, MD : Government Institute Inc., 1985. 
completely from aqueous solutions. Several decades of research and industrial experience have prover the technology. Process economics usually demand that the carbon be reused, however, and so the carbon must be regenerated. The goals of a regeneration process are to restore as much adsorption capacity as possible at the lowest possible cost. Investigators attempting to remove phenolic compounds from carbon have had widely varying degrees of success. Recpveries from 0 to 100 percent are raported in the literature. In industrial processes, regeneration by oxidation typically lowers the capacity of a carbon by 5-15t. These facts have led to considerable speculation about the nature of surface interactions with the sorbate. The studies cited in the remainder of this section illustrate the state of uncertainty about those interactions.

Suzuki et al. (1978) used thermal gravimetric analysis (TGA) to compare removals of several organic sorbates from an activated carbon. The organic compounds that were liquids at ambient temperature were adsorbed from the pure liquids onto carbon. After unspecified contact times the carbon particles were separated from the liquids and dried at room temperature. The solid compounds were dissolved in distilled water, and the resulting solutions were contacted with carbon. After the adsorptions, the carbon samples were separated from the solution's and dried overnight at 
$110^{\circ} \mathrm{C}$. A sample of each loaded carbon was placed in an inert atmosphere and the weight change was monitored as the temperature was raised from $100^{\circ} \mathrm{C}$ to $800^{\circ} \mathrm{C}$ at $6{ }^{\circ} \mathrm{C}$ per minute. Plots of loading divided by initial loading vs. temperature (TGA curves) were characteristic of the individual compounds.

These investigators classified the organic compounds into three groups according to the shapes of the TGA curves. Table 1.2 shows the groups. Group I organics are relatively volatile and lest the carbon at ralatively low temperatures. Regeneration was essentially complete, and an equilibrium model was used to describe the desorption. As shown in Table 1.2, several alkanes and alcohols, as well as benzene and some substituted benzenes, belong to Group I.

In contrast, Group II compounds were not completely desorbed. These compounds have relatively high boiling points and a fraction of each decomposed before desorption. Small amounts of residual organies remained on the carbon at $8000^{\circ}$. The authors modelled the TGA curves with a thermal cracking model and reasoned that the residue was either a carbon deposit or a polymerization product of by-products from the cracking reactions. In any case, at least part of the adsorption was not reversible under the conditions employed. 
Table 1.2 Classification of Organic Adsorbates by Suzuki et al. (1978)

\begin{tabular}{|c|c|c|c|}
\hline Adsorbate & Molecular Weight & Boiling Point o $\mathrm{C}$ & Organic Type \\
\hline n-Pentane & 72 & 36 & 1 \\
\hline n-Hexane & 86 & 69 & 1 \\
\hline n-Heptane & 100 & 98.4 & I \\
\hline n-Decane & 142 & 174 & I \\
\hline n-Pentadecane & 212 & 270 & I or II \\
\hline Cyclohexane & 84 & 83 & I \\
\hline Benzene & 78 & 80 & I \\
\hline Toluene & 92 & 110.6 & I \\
\hline p-Xylene & 106 & 114 & I \\
\hline Butanol & 74 & 118 & $\mathbf{I}$ \\
\hline Hexanol & 102 & 157.5 & I \\
\hline Octanal & 130 & 194.5 & I \\
\hline Coumarin & 146 & 290 & $I$ \\
\hline Benzophenone & 182 & 305.9 & II \\
\hline Vanillin & 120 & 285 & II \\
\hline Phenol & 94 & 182 & III \\
\hline$B \cdot$ Napthol & 144 & 285 & III \\
\hline Phloroslucinol & 126 & & III \\
\hline p-Hydroxydiphenyl & 170 & 308 & III \\
\hline $\begin{array}{l}\text { Sodium } \\
\text { dodecylbenzensulfonate }\end{array}$ & 326 & & II \\
\hline Tetracthyleneglycol & 194 & & II \\
\hline Polyethylenezlycol 400 & -400 & & II \\
\hline Polyethyleneglycol 1000 & -1000 & & II \\
\hline Polyethyleneglycol 4000 & -4000 & & II \\
\hline Benzaldehyde & 106 & 179.1 & $I$ \\
\hline p-Oxybenzaldehyde & 122 & 310 & III \\
\hline Lignin & & & III \\
\hline Humic acid & & & III \\
\hline Methylene blue & 320 & & III \\
\hline Benzoic acid & 122 & 250 & III \\
\hline Caproic acid & 116 & 205.4 & II \\
\hline Butyric acid & 88 & 164.1 & 1 \\
\hline
\end{tabular}


Group III compounds include phenol and several phenolic compounds, as well as benzoic acid. Carbon samples loaded with these compounds exhibited gradual decreases in weight as the system temperature increased, and the samples retained large amounts of residual organics at $800^{\circ} \mathrm{C}$. For phenol (and for polyethylene glycol, type II) the weight of residual was proportional to the initial loading of sorbate. It was also determined that regeneration of phenol was less faclle from a carbon containing a relatively large amount of ash.

The investigators decided that "aromatic content" and boiling point are the two main characteristics that influence the thermal desorption behavior of a compound. A plot oi 0 vs, boiling point, where

$$
\phi=\frac{\text { number of aromatic carbons in a molecule }}{\text { total number of carbons in a molecule }}
$$

was divided into three regimes, one regime corresponding to each group. The investigators reasoned that compounds falling into the group III regime would be refractory to thermal regeneration $0:$ a process scale.

Seewald et al. (1983) combined TGA with mass spectrometry to study the desorption of phenol and cyclohexane from activated carbon. Adsorption was 
isothermal at temperatures ranging from $25^{\circ} \mathrm{C}$ to $112^{\circ} \mathrm{C}$ from various media including helium, air, and aqueous solutions. Desorption into helium was achieved by raising the temperature from $25^{\circ} \mathrm{C}$ to $800^{\circ} \mathrm{C}$ at a constant but unspecified rate. Desorbed compounds were identified by passing the helium through a mass spectrometer.

The investigators found several 'peaks' in their desorption curves. They used the TGA curves to distinguish between a fraction of the sorbate that they believed was physisorbed and a eraction that they believed was "influenced by surface reactions". The "reacted" or chemisorbed material was classified further into desorbable and non-desorbable portions. The authors argued that the physically adsorbed solute was present in relatively large pores while the chemisorbed solute was located in smaller pores. They found only slight changes in pore size distribution due to non-desorbable residue, however. Although they did not determine the nature of the residue, they did refer to studies that indicate that the residue is more reactive with steam than was the original carbon.

The adsorption conditions affected the amount of "reacted" sorbate. Adsorption of phenol or cyclohexanone from air resulted in mure chemisorption than did adsorption from helium. In addition, while all of the phenol adsorbed from aqueous solution at $25^{\circ} \mathrm{C}$ was judged to be physisorbed, higher adsorption temperatures promoted chemisorption. 
Two desorbable renction products (thought to be formed during the adsorption contact) were identified by mass spectrometry. The investigators reported that the quantities of hydroxy-diphenyl ether and dibenzofuran formed depended on the adsorption temperature, the adsorption atmosphere, and the carbon pore structure. They rgued that dehydrogenation of cyclohexanone yields phenol, and reaction of phenol molecules yields the ether. Dibenzofuran may be formed through dehydration of hydroxy-diphenylether.

Robertacclo (1976) reported complete recovery of phenol from a powdered activated carbon. Adsorption and desorption contacts were with aqueous solutions buffered at pH 2 and held at $24^{\circ} \mathrm{C}$. Contact times ranged from ten minutes to two hours. Coincident adsorption and desorption isotherms were established over the range 35-200 $\mathrm{mg}$ of total organic carbon per liter.

In contrast, yonge et al. (1985) found 'hystoresis' in adsorption/desorption isotherms. Phenolic compounds were adsorbed onto carbon from aqueous solutions buffered at pH 7 , and then desorbed into buffer solutions that were initially solute free. Batch desorption contacts were made for each loaded carbon until no solute could be detected in the regenerant solution. Adsorption and desorption contacts were for 10 days at ambient temperature. 
Table 1.3 shows that large fractions of the phenoli= sorbates remained on the carbon after regeneration. The investigators argued that strong bonds formed between the sorbates and surface functionai groups and made the adsorption irreversible. They surmised that charge-transfer complexes could form with high enough bonding energles to resist regeneration. They also suggested that alkane and methoxy substituents on the phenolic sorbates intensified the binding energies and that the effect was greater for ortho substitution than Enr para substitu+ion.

Yonge et al. also found that the procedure used to develop an adsorption isotherm affected the loadings. Higher Ioadings were achieved when the same sample of carbon was used repeatedly to establish the isotherm points, than when separato samples were usad. In the former method, the carbon was contacted for ten days eash with solutions of increasing initial solute concentration. In the latter method, each sample was equilibrated with only one solution for ten days. The two types of isotherms shared the point corresponding to the lcwest concentration tested, and the investigiators argued that the isotherms shoujd converge when the surface was "saturated" with sorbate.

The researchers attempted to explain the divergence of the isotherms at intermediate concentrations by the 
Table 1.3

Desorption Irreversibility in the Study of Yonge et al. (1985)

Sorbate

Phenol

4-1sopropylphenol

2-Ethylphenol

o-Cresol

o-Methox yphenol
Irreversible Adsorption, \%

85

87

91

94

97 
development of radial concentration profiles in the particles. They argued that high concentrations of sorbate developed on pore surfaces near the outside of the particles during the initial stages of the adsorption contact, promoting high levels of irreversible adsorption. Inner surfaces would develop much lower loadings because they contacted only solutions that were depleted of solute. This argument requires that the reaction responsible for irreversible adsorption be rapid under the conditions studied.

Finally, these investigators argued that radial profiles could be eliminated and true equilibrium isotherms established by continually contacting the carbon with a solution of fixed sorbate concentration. Continuous flow through a packed bed was used to approach this condition. Contact continued until the influent and effluent concentrations were equal. The investigators expected to achieve higher loadings with this method (due to higher concentrations in the inner pores) but measured lower loadings than for the other two methods.

Zogorski and Faust (1978) developed isotherms for the adsorption and desorption of 2,4-dichlorophenol at $25^{\circ} \mathrm{C}$. In contrast to the findings of yonge, they found that the desorption branch of the isotherm fell slightly below the adsorption branch. For adsorption, aqueous solutions buffered at pH 6.3 were contacted with carbon for 12 to 14 
days. Several batch contacts of the loaded carbon with distilled water were used to establish the desorption isotherm. The investigators argued. that the differences in the isotherms were due to small experimental errors and that the adsorption of phenols is, in general, a completely reversible process.

Zogorski and Faust also developed isotherms for the adsorption of phenol onto carbon at 8,20 , and $29^{\circ} \mathrm{C}$. The amount of phenol adsorbed from aqueous buffer solutions at pH 6.3 decreased with increasing temperature, as expected for an exothermic (physisorption) process.

Fuerstenau (1984) found a different trend for adsorption of o-cresol onto coal. He found that both the rate and the amount of adsorption increased with increasing temperature. The temperature was varied from $10^{\circ} \mathrm{C}$ to $50^{\circ} \mathrm{C}$, and contact times up to nine days were used.

Goto et al. (1986) loaded phenol onto a bed of activated carbon and onto a bed of strong-base anion exchange resin and regenerated the adsorbents with IN NaOH. The beds were maintained at $30^{\circ} \mathrm{C}$, and adsorption and desorption contacts were for less than 8 hours each. While 1007 recovery from the resin was reported, snly 70 of the phenol was recovered from the activated carbon. Repetition of the adsorption and regeneration processes for several cycles resulted in a gradual decrease in capacity of the carbon, but an unchanging capacity of the resin. 
Finally, Thakkar and Manes (1985) found that the phenolic compounds they tested could be quantitatively recovered, and "do not appear to react with activated carbon". They sorbed various compounds onto carbon from methylene chloride with contact times greater, than 48 hours. The loaded carbon was contacted with a mixture containing a phenolic 'displacer' in methanol for up to 7 days. The amount of the displacer was far in excess of the amount of original sorbate so that the law of mass action favored desorption of the orlginal sorbate. The authors concluded that the apparent irreversibility of adsorption observed in some earlier investigations was due to inadequate regeneration methods. In particular, they argued that organic solvents could not interact as energetically with surface functional groups as could phenolic displacers.

These studies illustrate the current state of confusion about the interactions of phenolic compounds with activated carbons. The key issues include the chemical nature and the degree of reversibility of those interactions. The properties of activated carbons, of sorbates, and of the adsorption and desorption media all seem to affect regenerability. The present work was dirested at resolving these matters. 


\section{References}

Fuerstenau, D.W. Seminar on the Surface Properties of coal and It's Elotation, Technical University of Clausthal, west Germany, June, 1984 .

Goto, M.: Hayashi, N.; and Goto, s. Environ, Sci, Technol., $1986,20,463-467$.

Robertaccio, F.L., Ph.D. Thesis in Environmental Engineering, University of Deleware, 1976.

Seewald, H.: Klein, J.; and Juntgen, H., "Correlation Between Desorption Behavior and Adsorption Characteristics of Organic Substances on Activated Carbon", Eundamentals of Adsorption: Procesdings of the Enginenting Eoundation conference held at sehloss Elmau, Bavaria, West Germany, May 6-11, 1983, eds. Meyers, A.I., and Belfort, G., Engineering Foundation, New York, 557-565, 1983.

Suzuki, M.; Misic, D.M.: Koyama, O.: and Kawazoe, K. Shem. Eng. Sei,, 1978, 33, 271-279.

Thakkar, S. and Manes, M. Environ, Sci, Technol, 1987, 21, 546-549.

Yonge, D.R.; Keinath, T.M.; Poznanska, K.; and Jiang, Z.P. Environ, Sci, Technol, 1985, 12, 600-694. 
Zogorski, J.S. and Faust, S.D., "Equilibria of Adsorption of Phenols by Granular Activated Carbon", Chemistry of Wastewater Technology, ed. Rubin, A.J., Ann Arbor Science Publishers, Ann Arbor, Mich, 143-160, 1978. 
Chapter 2 Evidence for a Chemical Reaction

As speculated in Chapter 1, a potential cause of irreversible adsorption is reaction of the sorbate with or on a carbon surface. An early step in this work was to determine if such a reaction occurs during adsorption. At the outset, we were faced with the probability that this type of reaction would be difficult to characterize, since presumably the products would be tightly bound to the surface. Even if reaction products could be recovered, they might have been formed during, or be altered by, the desorption process. These concerns lead us to focus initially on the adsorption process. By comparing rates and amounts of adsorption it was established that chemical reaction accompanied the adsorption of phenol onto activated carbon.

2.1 sources, Pretreatment, and surface Areas of Adsorbents

Several granular activated carbons and several polymeric materials were used in this research. Table 2.19 lists the commercial source of each carbon and indirates the raw materials from which the carbons were derived. Table 2.1b shows the commercial source and the compositions of the polymeric adsorbents. 
Table 2.1a Vendor-Supplied Activated Carbons Used in This Study

\begin{tabular}{lccc}
$\begin{array}{l}\text { Adsorbent } \\
\text { Designation }\end{array}$ & Vendor & $\begin{array}{c}\text { Raw } \\
\text { Material }\end{array}$ & $\begin{array}{c}\text { N2-BET } \\
\text { Surface Area } \\
\left(\mathbf{m}^{2 / g d c)}\right.\end{array}$ \\
\hline Witcarb 950 & Witco Chemical Corp. & Petroleum & 1050 \\
Super-50rb. GX031 & Amoco Research Corp. & Petroleum & 2350 \\
Norit Row 0.8s & American Norit Co. & Peat & 650 \\
Filtrasorb-100 & Calgon Carbon Corp. & Coal & 800 \\
Columbia & Witco Chemical Corp. & Petroleum & 900 \\
\hline
\end{tabular}

Table 2.Ib Polymeric and Pyrolyzed-Polymer Adsorbents Used in This Study

Adsorbent

Designation*

XAD-4

XAD-7

$\mathrm{XE}=\mathbf{3 4 0}$

XE-348

IRC-50
Type of Adsorbent Styrene-Divinylbenzene Copolymer Acrylic ester-Divinylbenzene Copolymer

Pyrolyzed Sulfonated Styrene-Divinylbenzene Copolymer

Pyrolyzed Sulfonated Styrene-Divinylbenzene Copolymer

Weakly Acidic Cation Exchanger Methacrylic Acid-Divinylbenzene Copolymer
$\mathrm{N}_{2}$-BET Surface Area (m²/gda)

725

450

400

$500 *$

ND**

40

ND $\cdots$

"These adsorbents are manufactured by Rohm and Haas Company.

- Manufacturers stated value.

-Not Determined: Approximate value $20-40 \mathrm{~m}^{2} / \mathrm{gda}$; personal communication G.R. Ackermann, Rohm and Haas Company.

".Not Determined: Approximate value $300 \mathrm{~m} 2 / \mathrm{gda}$; Technical bulletin Rohm and Haas Ion Exchange Resins and Eluid Process Chemicals for Special Applications Rohm and Haas Company, 1986. 
The first three carbons in Table 2.1 a were rinsed several times in Milli-Q water and dried at $98^{\circ} \mathrm{C}$ under 24 in. Hg vacuum for at least 48 hours. Each of the remaining adsorbents was extracted with methanol in a soxhlet apparatus for three hours, rinsed with acetone, rinsed with waterl, and then extracted with water in a soxhlet apparatus for three more hours. A final rinse with water was used to remove any fines. The sorbents were then dried at $98^{\circ} \mathrm{C}$ (carbons) or $80^{\circ} \mathrm{C}$ (polymeric adsorbents) under 24 in. Hg vacuum for at least 48 hours. The materials were removed from the drying oven, allowed to cool in a dry nitrogen atmosphere, and sealed into glass bottles in that atmosphere.

The extraction procedure served to ensure that future contact between an adsorbent and water, methanol or acetone would have minimal effect on the surface properties of the adsorbent. Handling in a nitrogen atmosphere prevented contact of adsorbents with oxygen or moisture after these substances were removed from the drying oven. Properties of the sorbents pre-treated by these methods are discussed in Chapter 4.

1 Inorganic ions and organic contaminants were removed from water used in this work by double-distillation followed b: adsorption onto Millipore Milli-Q filters. The water passed through a prefilter, an activated carbon bed, two beds containing a mixture of strong acid and strong base ion-exchange resins, and two cellulose acetate membranes (to remove microscopic particles and microorganisms. 
Dry resins that would not wet with water at $25^{\circ} \mathrm{C}$ were pre-wet with methanol at room temperature and then rinsed with water until no methanol could be detected by gas chromatography of a rafinate sample. These resins were then centrifuged to remove interstitial water. Resin samples were placed in 15-ml, medium-frit glass filters that were in turn, placed in polymeric centrifuge tubes and covered with Parafilm to prevent evaporative losses. Centxifugation was accomplished with a Damon/IEC model CL centrifuge operated at $2500 \mathrm{rpm}$ for 10 minutes. A fraction of the centrifuged particles was weighed, dried in a vacuum oven for 48 hours, and then re-weighed to determine the relative weights of dry resin and water in pre-wet samples.

Tables $2.1 \mathrm{a}$ and $b$ also list surface areas for the various adsorbents. Surface areas were determined according to the Nitrogen-BET method. The method is widely used and is discussed in many texts (e.g. Mikhail and Robens, 1983). Nitrogen adsorption data were measured with a Model 201 BET Analyzer (Porous Materials, Inc.). Samples were outgassed at $200^{\circ} \mathrm{C}$ for approximately 90 minutes and under vacuum. The sample chamber was then contacted with liquid nitrogen, and a pressure close to 20 microns $\mathrm{Hg}$ was attained before adsorptic.s measurements were made. Data taken for relative pressures ( $P$ /Psat, where psat is the saturation pressure of nitrogen at $77.2 \mathrm{~K}$ ) between 0.02 and 0.2 were used to calculate surface areas from the 
linearized form of the BET equation. Significant deviations from linearity were observed at higher relative pressures.

\subsection{Determination of Adsorption Amounts and Isotherms}

Each point on an adsorption isotherm was established by contacting a known mass of adsorbent with a known mass of a solution containing a solute/sorbate. Most often the solution was aqueous. The contact vessel was sealed and maintained at a constant temperature with an oil, water, or air bath. Agitation was provided with either a magnetic stir bar or a mechanical shaker. The concentration of solute in the liquid at the end of a contacting period was measured by gas chromatography (GC) or by high performance liquid chromatography (HPLC). A detailed explanation of these tachniquas appears in Appendix I.

Equation 2.1 is a mass balance comparing initial and final conditions for a batch adsorption contact where the liquid is a binary mixture (Jaycock and Parfitt, 1981).

$$
\frac{n^{0}\left(x_{i}^{0}-x_{1}\right)}{m}=n_{1}^{*} x_{2}-n_{2}^{3} x_{1}
$$

The group of terms on the left hand side is known as the "composite uptake" of the adsorbing species. The variable $n^{\circ}$ is the total number of moles of liquid components 
introduced to the contacting vessel, $x_{1} \circ$ is the initial mole fraction of solute in the liquid, $x_{1}$ is the final mole fraction, and $m$ is the mass of the adsorbent in grams. The composite uptake is an apparent loading based on depletion of the solute from the liquid phase. The term $n_{1} s$ on the right hand side is the sum of the loading of sorbate on the adsorbent surface and the amount of solute held in liquid in the pores, and is termed the "individual uptake" of the solute. The variable $\mathrm{n}_{2} \mathrm{~s}$ is the individual uptake of the solvent. The composite uptake is a useful quantity because it is should be a thermodynamic property. The individual uptake gives the total solute uptake by the sorbent, but requires a definition of a division between the surface phase and the bulk liquid. That division is obtained by centrifugation in this work.

The difference between individual and composite uptakes is small when the selectivity of sorption is high, when the amount of solvent taken up by the sorbent is relatively small, and/or when the solute is extremely dilute in the solvent throughout an adsorption contact. For dilute solutions, the final mole fraction of the solvent $\left(x_{2}\right)$ is almost unity, the final mole fraction of the solute is almost zero, and equation 2.1 becomes:

$$
\frac{n^{0}\left(x_{i}-x_{1}\right)}{m}=n_{i}
$$


Equation 2.2 applies to almost all of the experiments reported in this work, and so a measured composite uptake can be interpreted as the surface loadin;;.

Composit adsorftion "isotherms" were established by contacting a sorbent, a solute, and a solvent in different proportions in a number of contact vessels exposed to the same external environment (same temperature and mode of agitation). A plot of composite adsorption vs. final solute concentrations or activities is an isotherm but may not be an equilibrium isotherm. It will be demonstrated that irreversibilitios can hinder the attainment of thermodynamic equilibrium.

\subsection{Comparison of Liquid-vapor and solld-Liquid Burface Excesses}

When the composite adsorption is divided by the specific surface area of the solid it is termed the "surface excess" of the sorbate. Figure 2.1 shows surface excesses of phenol at three types of interfaces involving aqueous solutions. The ordinate is the activity of phenol, which is the product of the solute mole fractinn and an activity coefficient (discussed below) ${ }^{2}$. Filtrasorb-100 $(F-100)$ is an activated carbon derived from coal, and XAD-4 is a polystyrene-divinylbenzene copolymer. Various amounts of these adsorbents were contacted with $15 \mathrm{ml}$ aliquots of

2 All activity values in this dissertation have units mole of solute per mole of solution. 


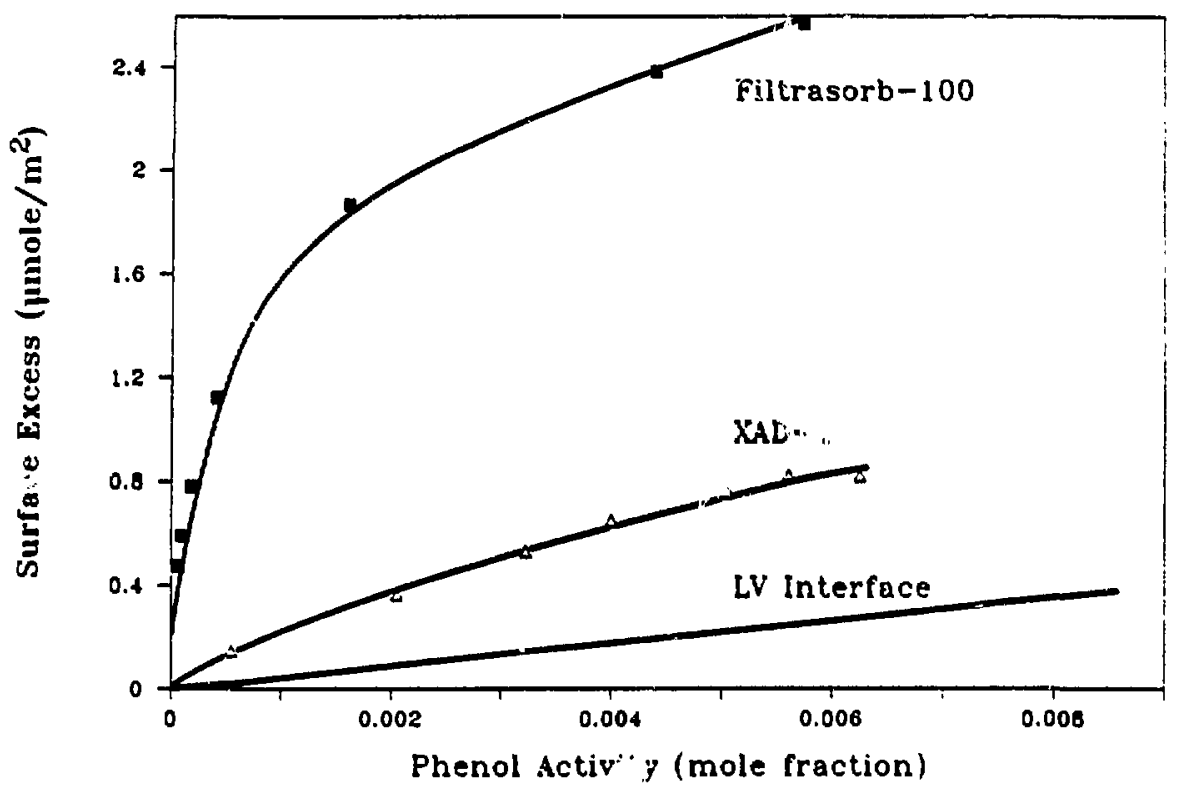

Figure 2.1 Surface Excesses of Phenol at Interfaces with Aqueous Solutions at $25 \cdot \mathrm{C}$. 
phenol-water mixtures in $20 \mathrm{ml}$ scintillation vials for 10 days at $25^{\circ} \mathrm{C}$. The vials containing F-100 ( $100 \times 140 \mathrm{mesh}$ ) were stirred vigorously, while the vials containing XAD-4 (20x40 mesh) were agitated by mechanical shaking. As will be seen. mass transfer limitations did not limit the uptakes onto XAD-4. The curves associated with the data points are not predicted, but are meant to show the general shapes of the isotherms.

The curve for surface excesses at liquid-vapor (IV) interfaces is predicted. The composite adsorption can be related to the rate of change of liquid-vapor surface tension with changing chemical potential of the solute through a form of the Gibbs Equation (Adamson, 1982):

$$
-d \sigma^{2 v}=\Gamma_{1} d \mu_{1}+\Gamma_{2}^{*} d \mu_{2}
$$

Here $\sigma^{\text {tv }}$ is the liquid-vapor surface tension, and $\mu$, is the cheraical potential of component $i$ in a binary mixture. The variable $r$ : is the excess number of moles of species $i$ at the interface per unit area. The Gibbs-Duhem Equation for three dimensional phases with two components at constant temperature and pressure is (Smith and Van Ness, 1975):

$$
x_{1} d \mu_{1}+x_{2} d \mu_{2}=0
$$

Combining the previous two equations gives: 


$$
-d \sigma^{L V}=\Gamma_{1}^{s} d \mu_{1}-\Gamma_{2}^{s} \cdot \frac{x_{1}}{x_{2}} d \mu_{1}
$$

or

$$
-x_{2} \frac{d \sigma^{2 v}}{d \mu_{1}}-\Gamma_{1} x_{2}-\Gamma_{2}^{*} x_{1}
$$

Dividing equation 2.1 through by the specific surface area - and combining with equation 2.5 yields;

$$
n \cdot \frac{\left(x i-x_{1}\right)}{m s}=-x_{2} \frac{d \sigma^{2 v}}{d \mu_{1}}
$$

For a liquid-vepor interface, the left-hand-side denominator is considered a single variable; ms = intertacial area.

Data for surface tension vs. solution composition for phenol in water at $20^{\circ} \mathrm{C}$ are available in the International Cricical rables (Washburn, 1928) and were used to evaluate the righ: hand side of equation 2.6. The activity coefficient for phenol $(N)$ was assumed to be constant and equal to the value at infinite dilution for the very dilute solutions represented in Figure 2.1 (see Appendix 2). Under these conditions:

$$
\mu_{1}=\mu_{1}^{0}+R T \ln \left(f_{1} x_{1}\right)
$$




$$
\begin{gathered}
d \mu_{1}=R T d \ln \left(f_{1} x_{1}\right) \\
d \mu_{1}=R T\left[d \ln (f)+d \ln \left(x_{1}\right)\right] \\
d \mu_{1}=R T d \ln \left(x_{1}\right)=\frac{R T}{x_{1}} d x_{1}
\end{gathered}
$$

Equation 2.7 with the substitution $T=298 \mathrm{~K}$ was used to evaluate the derivative in equation 2.6. Changes in surface tension with solute mole eraction should be similar at $20^{\circ} \mathrm{C}$ and at $25^{\circ} \mathrm{C}$ (see Worley, 1914).

Equation 2.6 shows that decreasing surface tension with increasing chemical potential of phenol is a driving force to concentrate phenol at a liquid-vapor interface. The same driving force concentrates phenol at solid-liquid interfaces, but, in addition, specific interactions of the liquid components with the solid may affect the surface excesses. The fact that the composite isotherm for XAD-4 lies above the line for a liquid-vapor interface indicates that phenol has more affinity for the solid than does water. In contrast, Rixey (1987) found a close correspondence between surface excesses of butyric acid and acetic acid on $X A D-4$ and surface excesses at liquid-vapor interfaces. 
Phenol has relatively more affinity for XAD-4 than does water, but the difference in affinities is not nearly as great for XAD-4 as it is for F-100. Phenol interacts very strongly with the activated carbon, and the surface excesses are almost an order of magnitude greater on F-100 than at a liquid-vapor interface throughout the activity range compared. This comparison shows that specific interactions of phenol with activated carbon dominate the adsorption behavior. The comparison does not prove that a chemical reaction occurs, but it does show that the properties of the sorbate and of the surface influence the surface excesses more than do surface tension and solution-phase activity effects.

Figures $2.2 \mathrm{a}$ and $2.2 \mathrm{~b}$ provide further evidence for specific solute-surface interactions. Figure 2.2 a compares surface excesses of phenol and resorcinol at liquid-vapor interfaces and on XAD-4 as functions of the solute mole fractions in the aqueous phase ${ }^{3}$. At the same mole fraction, phenol has a greater tendency to concentrate at the non-interacting and the weakly interacting interfaces. Figure $2.2 b$ shows that the relative tendencies are reversed for adsorption onto Filtrasorb-100, after 35 days of

3 The curve for surface excesses of resorcinol at the aqueous phase-vapor interface was established in the same manner as the curve for phenol, with equations 2.6 and 2.7 and surface tension vs. composition data from the International Critical Tables (Washburn, 1928). 


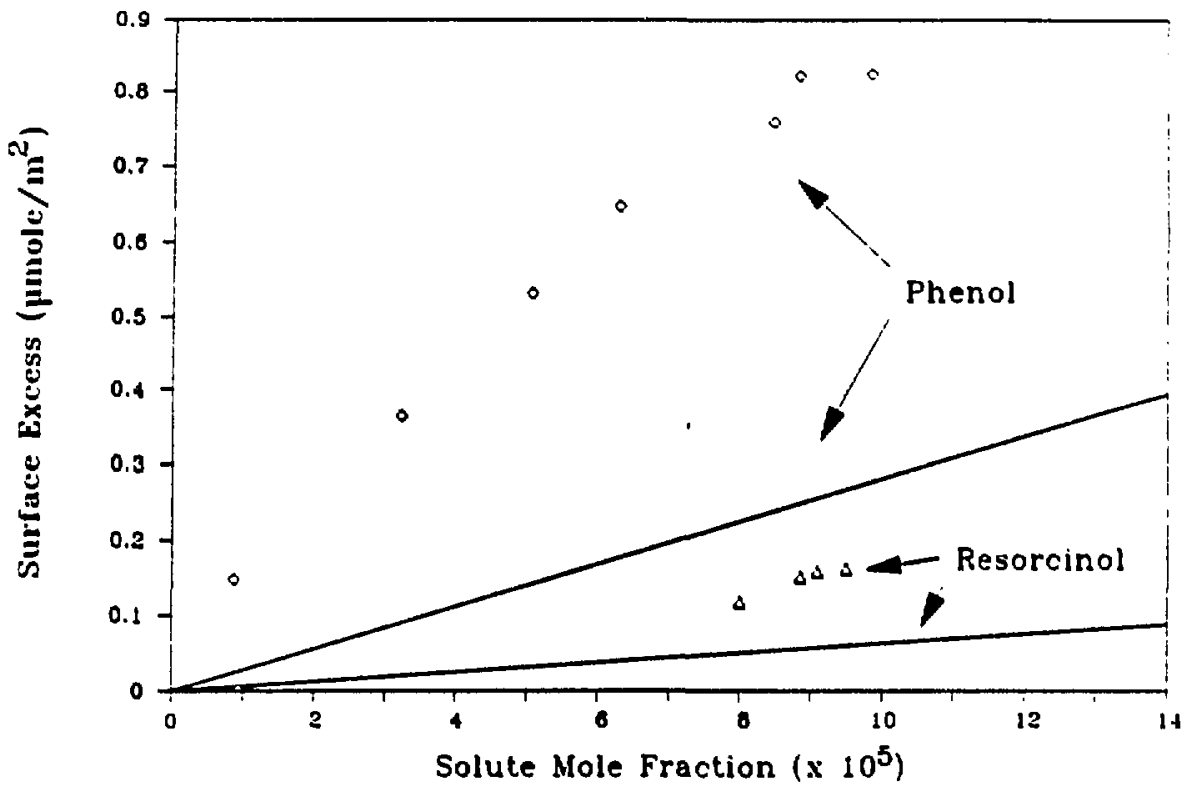

Figure 2.2a Surface Excesses of Phenol and Resorcinol at Interfaces with Aqueous Solutions: Vapor (Solid Lines) and XAD-4 (Symbols). 


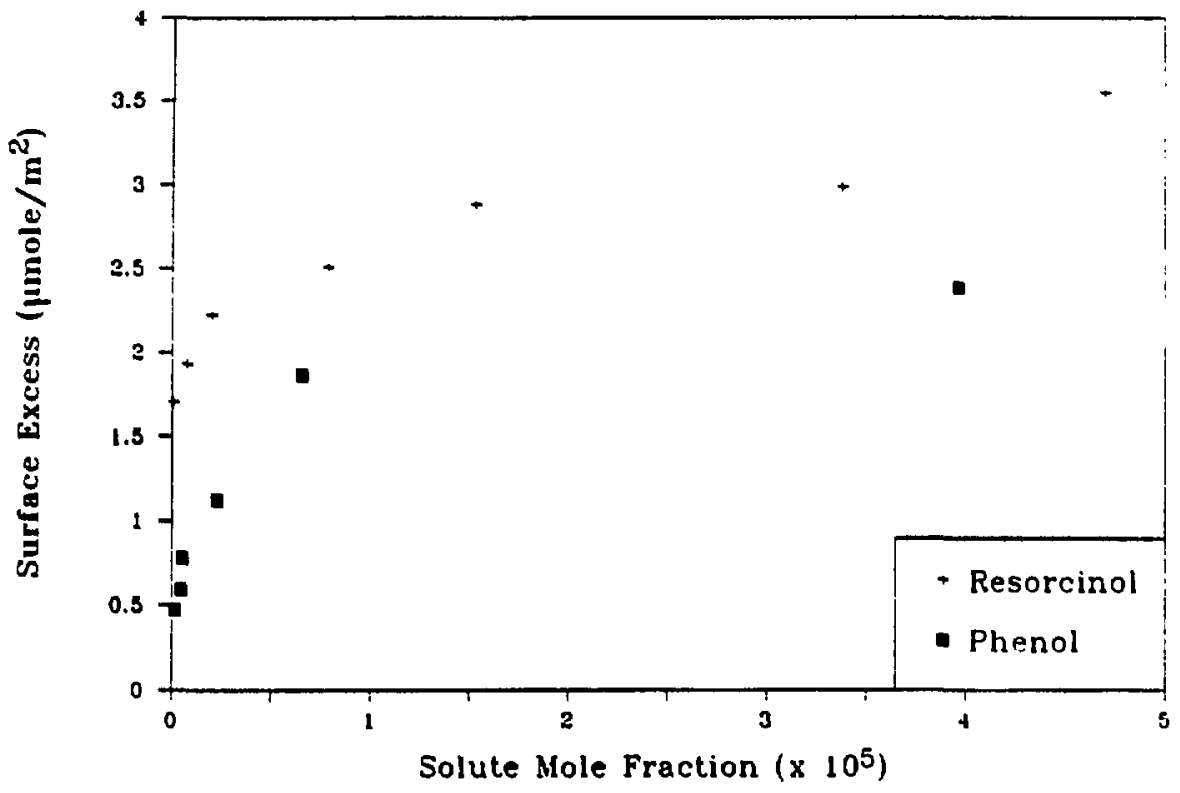

Figure 2.2b Surface Excesses of Phenol and Resorcinol on F-100 Carbon. Contact was for 35 Days at $25^{\circ} \mathrm{C}$. 
contact at $25^{\circ} \mathrm{C}$. Adsorption of the dihydroxybenzene onto the activated carbon is enhanced more by specific interactions than is adsorption of the monohydroxybenzene.

\subsection{Rates of Adsorption onto filtrasorb-100 Activated Carbon}

As illustrated in Chapter 1, contact times used to establish adsorption "equilibrium" between phenolic compounds and carbon vary widely between investigations. This variation reflects differences in the mass-transfer rates in different systems as well as the accuracy of measurements of solute concentrations and the tenacity of the experimentors. Generally, investigators monitor the solute concentration in a representative trial until the concentration reaches a value that does not change significantly between samples. A margin of safety is then added to establish a contact time for subsequent trials. Experimental results discussed in this section demonstrate the problems with this practice.

Figure 2.3 compares the kinetics of adsorption of ethanol, resorcinol, and phenol, onto $10 \times 20$ mesh $F-100$. For each of the solutes, $200 \mathrm{ml}$ of a dilute solution was introduced to a $250 \mathrm{ml}$ erlynmeyer flask containing the carbon. The initial solution concentrations and carbon weights were respectively; $1.72 \times 10^{-2} \mathrm{M}$ and $2.0 \mathrm{~g}$ for ethanol, $5.45 \times 10^{-3} \mathrm{M}$ and $0.33 \mathrm{~g}$ for resorcinol, and 


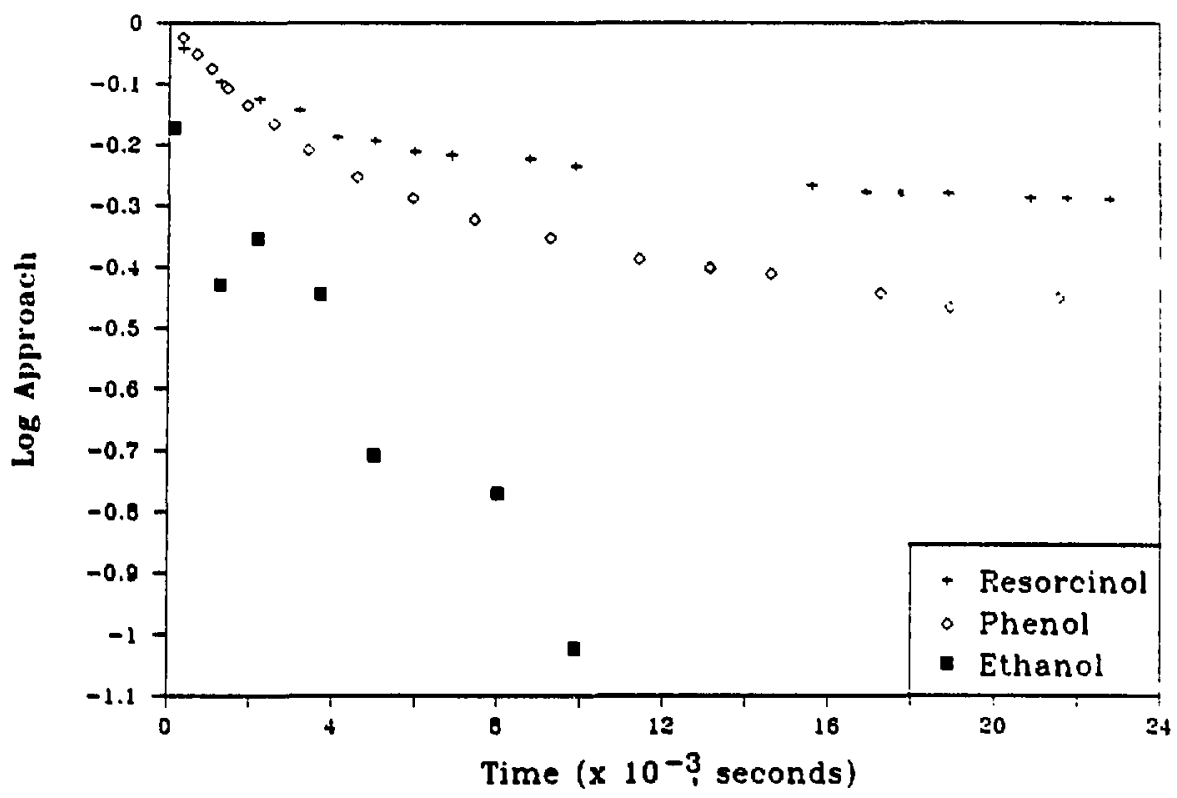

Figure 2.3 Kinetics of Adsorption for Three Sorbates on 10x20 Mesh F-100 Carbon. Approach to "Equllibrium" as a Function of Time. 
$6.38 \times 10^{-3} \mathrm{M}$ and $0.33 \mathrm{~g}$ for phenol. Each vessel was sealed with a rubber septum 4 , and was agitated at $1500 \mathrm{rpm}$ and maintained at $25^{\circ} \mathrm{C}$ in an orbital shaker bath (Lab-line Instruments Inc., Catalog no. 3535). Samples of volume 2 ml were withdrawn periodically through a sterilized needle into a glass syringe. The samples were rnalyzed by HPLC (for phenol and for resorcinol) or by GC (for ethanol).

The ordinate of Figure 2.3 is the base-ten logarithm of the approach to "equilibrium":

$$
\log \left(\frac{m_{1}-m_{1}}{m_{1}-m_{0}}\right)
$$

The letter m denotes the amount of solute adsorbed per gram of dry carbon (the composite adsorption), while the subscripts represent the time at which the measurements were made $(0$ denotes initial, $t$ denotes a particular time, and 1 denotes very long time). The $m_{1}$ values were determined after 11 weeks of contact.

The steeper the slope of a curve in Figure 2.3, the faster the approach to the final adsorption uptake. Ethanol adsorbed more quickly than did the phenolics throughout the trials. Further, the ethanol concentration reached a steady value after 8 hours of contact. The

4 In retrospect, a different method of sealing the flask should have been used, because organics can sorb into rubber. 
phenol and resorcinol concentrations changed slowly for about 8 and 10 weeks respectively, but after that time any changes were too small to detect in samples taken weekly. A slower approach to a final adsorption amount indicates an additional or a greater resistance to the attainment of equilibrium. These data show that there is more resistance for resorcinol than for phenol, and more resistance for phenol than for ethanol. If the extra resistance is due to chemical reaction, one might expect the reactivities of the solutes to follow the same order.

In order to establish the isotherms appearing in Figures 2.1 and $2.2 b$, Filtrasorb-100 carbon was ground with a mortar and pestle and was seived to $100 \times 140$ mesh size in order to reduce the mass-transfer resistance in adsorption contacts. The seived carbon was redried in a vacuum oven at $100^{\circ} \mathrm{C}$ and then cooled in nitrogen just before use.

Figure 2.4 compares the two isotherms for adsorption of phenol from aqueous solution onto the small particles. Each data point represents the results of contacting $15 \mathrm{ml}$ of a $6.38 \times 10^{-3} \mathrm{M}$ solution with a weight of carbon between 10 and $250 \mathrm{mg}$ in a $20 \mathrm{ml}$ scintillation vial fitted with a teflon cap liner. The vials were maintained at $25^{\circ} \mathrm{C}$ and stirred with magnetic stir bars at a rapid rate. The concentration of phenol in each vessel was monitored periodically by GC. After 10 days, no changes could be detected on an hourly basis. The isotherm measure. ifter 


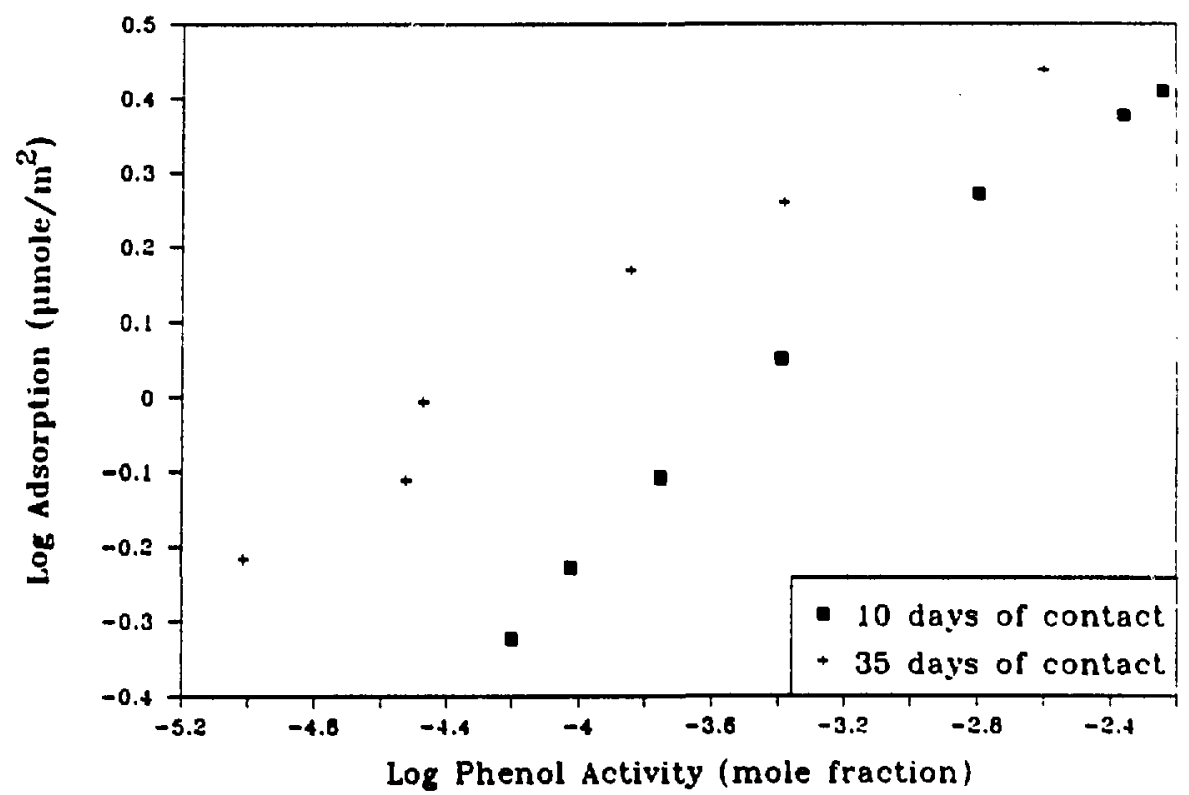

Figure 2.4 Adsorption of Phenol from Water onto F-100 Carbon at 25'C for 10 Days and for 35 Days. 
35 days of contact was established with separate trials that were sampled less frequently than were the trials used to establish the 10-day isotherm. This sampling proceedure was followed in order to lessen the risk of biological contamination. Adsorption densities increased substantially with time even after 10 days of contact in well agitated vessels. At these long times, mass-transfer rates should not be limiting the overall adsorption rate.

For comparison, Figures $2.5 a b$ show that cthanol ant 5,5-dimethylhydantoin reached steady adsorption amounts within two days in trials with similar contact conditions. This fact suggests that, for adsorption of phenol at $25^{\circ} \mathrm{C}$, there is a process that slowly increases the carbon loading long after equilibrium is established for physical adsorption. This is a hallmark of chemisorption or a chemical reaction.

\subsection{InEluence of Temperature on Adsorption Amounts}

Additional evidence for a chemical reaction between phenol and Filtrasorb-100 comes from a comparison of isotherus established after 1.0 days of contact at various temperatures. Figure 2.6 includes the "10-day isotherm" from Figure 2.4 as well as isotherms established at higher temperatures, but with otherwise identical contact conditions. Figure 2.6 shows that higher temperatures gave higher loadings for identical solution-phase phenol 


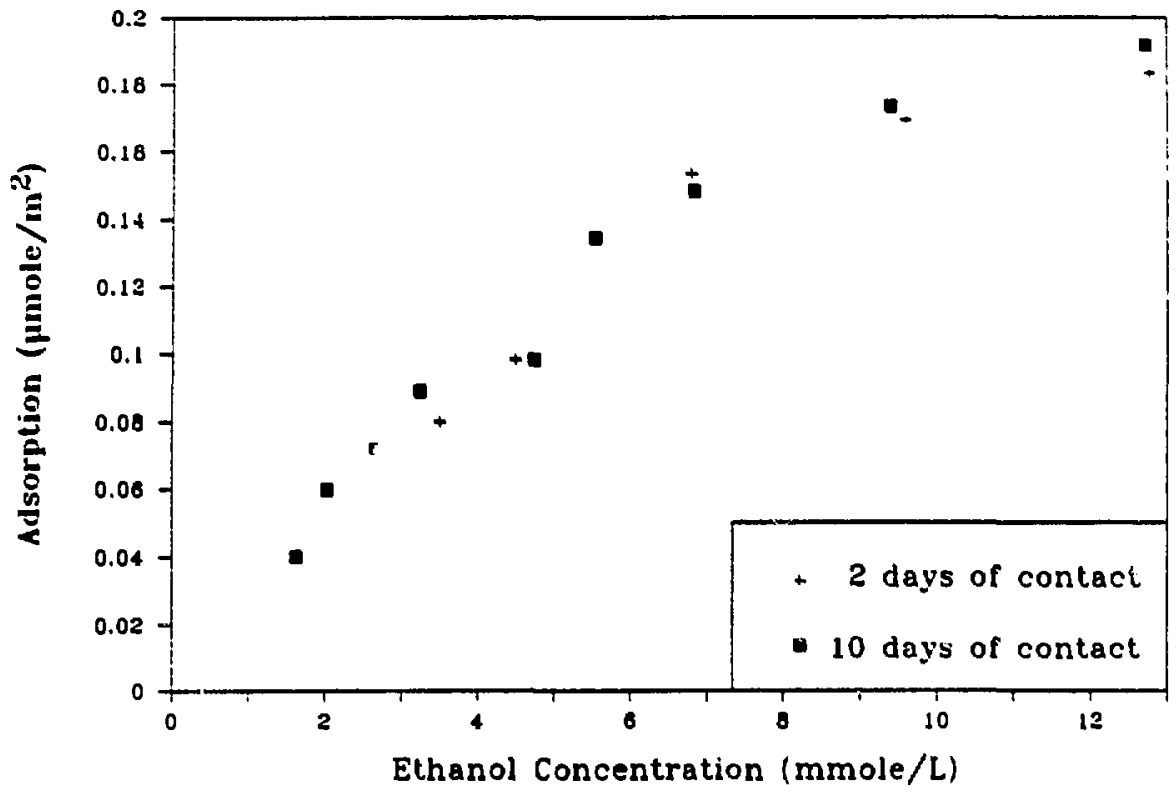

Figure 2.5a Adsorption of Ethanol from Water onto F-100 Carbon. 


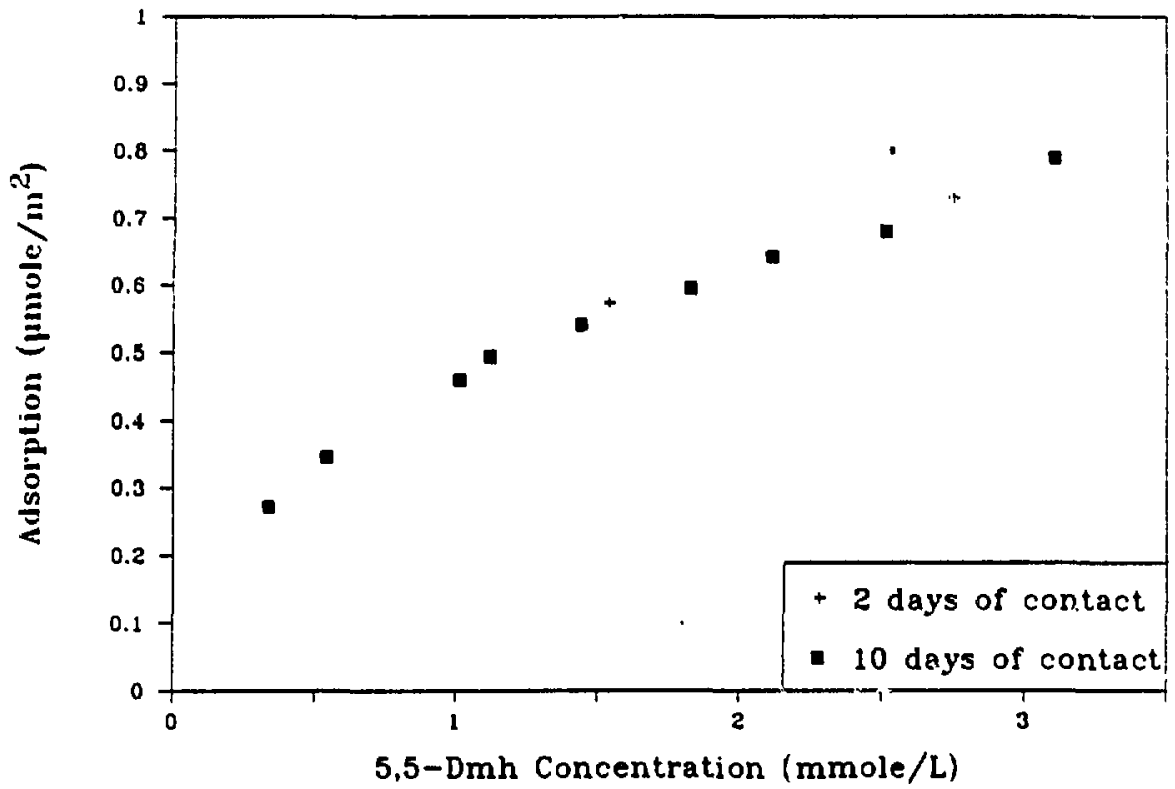

Figure $2.5 \mathrm{~b}$ Adsorption of 5,5-Dimethylhydantoin from Water onto F-100 Carbon. 


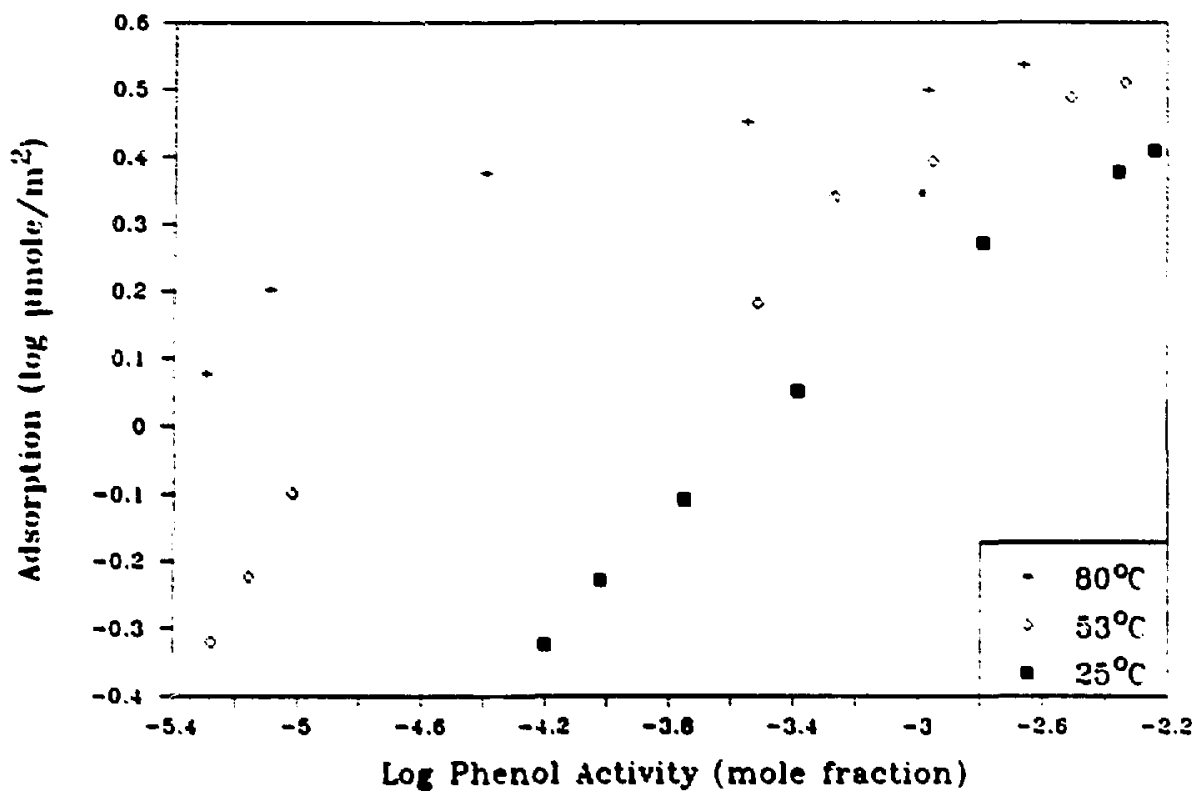

Figure 2.6 Adsorption of Phenol from Water onto F-100 Carbon at Various Temperatures. Contacts were for 10 Days at the Indicated Temperatures. 
activities (see Appendix 2 for infinite dilution activity coefficients at the different temperatures). In contrast, Figure 2.7 shows that isotherms for phenol adsorbed from water onto $X A D-4$ at $80^{\circ} \mathrm{C}$ and at $25^{\circ} \mathrm{C}$ are nearly coincident when normalized for activity.

At a given activity, a higher loading indicates more ensagetic interactions at the interface (see Appendix 2 and the discussion of the Lundelius rule and Traube's rule), so higher temperatures change the nature of the adsorption of phenol onto F-100, but not the nature of the adsorption of phenol onto XAD-4. It is also important to note that the isotherms in Figure 2.7 represent equilibrium conditions 5 , while those in Figure 2.6 do not. In fact, cooling mixtures used to establish the $80^{\circ} \mathrm{C}$ isotherm in Figure 2.6 to $25^{\circ} \mathrm{C}$ (in vigorously stirred vessels) resulted in slightly increased uptakes and a curve that lay substantially above the $25^{\circ} \mathrm{C}$ isotherm, as shown in Figure 2.86. These facts strongly suggest that F-100 promotes a chemical reaction which proceeds at a rate that increases with temperature.

5 The points on the $80^{\circ} \mathrm{C}$ isotherm were established in two different ways. The two points at the highest activities represent the results of contact.s for 5 days with no agitation. The remaining four points were established after 10 days of contact with agitation by magnetic stirring. of these later four points, the one corresponding to a phenol activity of $2.4 \times 10^{-3}$ was tested after 5 days and had the same loading as after 10 days.

- Physisorption is favored at lower temperatures, although physisorption equilibrium was probably not achieved over the two to three hour cooling period. 


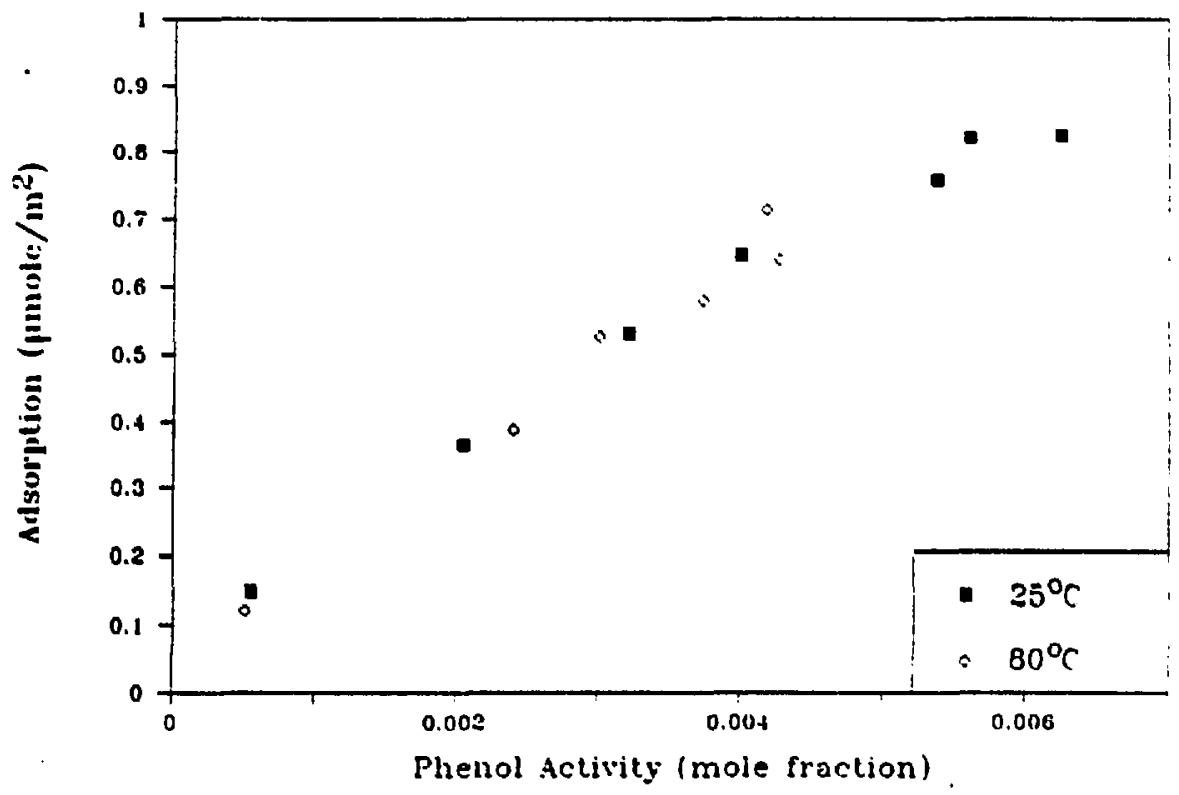

Figure 2.7 Adsorption of Phenol from Water onto XAD-4 at $25^{\circ} \mathrm{C}$ and at $8^{\circ} \mathrm{C}$. 


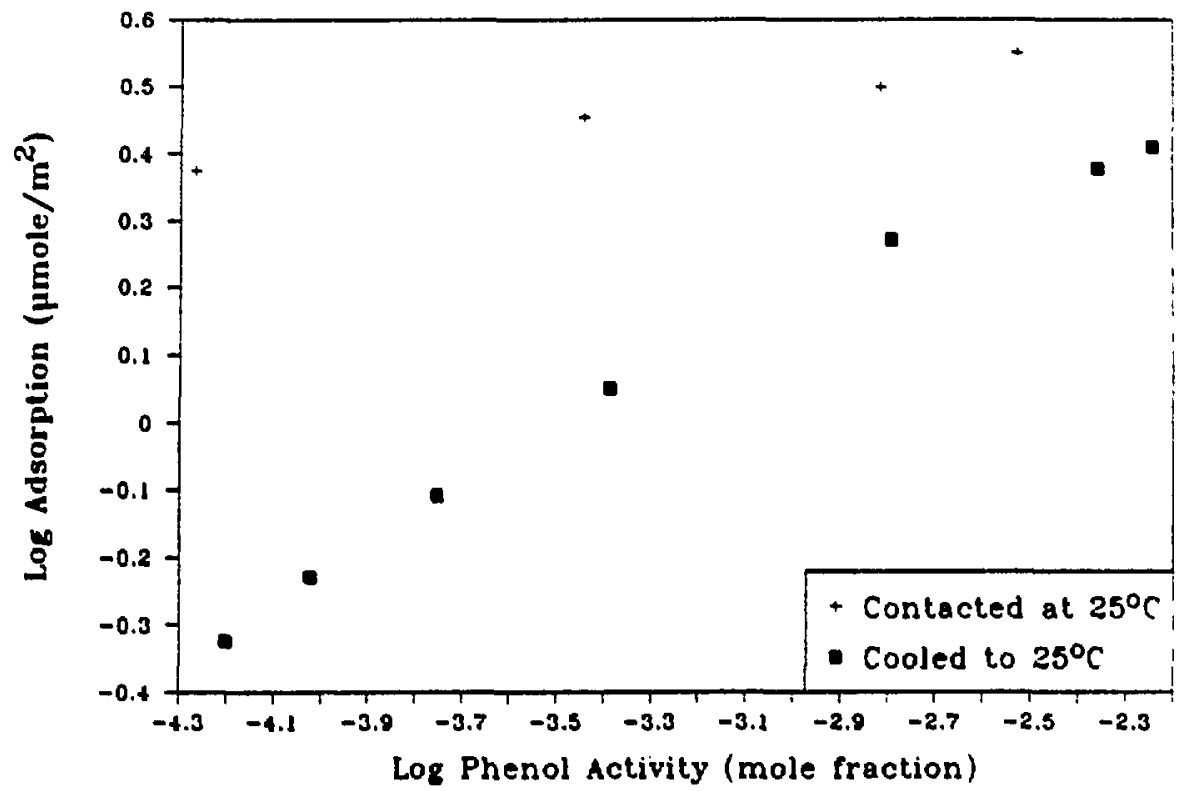

Figure 2.8 Adsorption of Phenol from Water onto F-100 Carbon for 10 Days. Contacts were at $25^{\circ} \mathrm{C}$ or at $80^{\circ} \mathrm{C}$ Followed by Cooling to 25.C. 
2.6 References

Adamson, A.w. Physical Chemistry of Surfaces, 4 th ed., Wiley-Interscience, NY, 1982.

Mikhail, R.S. and Robens, E. Microstructure and Thermal Analysis of solid surfaces, John Wiley \& Sons, NY, 1983. Jaycock, M.J. and Parfitt, G.D. Chemistry of Interfaces, Ellis Horwọod Iimited, Chichester, England, 1981.

Rixey, W.G., Ph.D. Dissertation in Chemical Enginearing, University of California, Berkeley, 1987.

Smith, J.M. and Van Ness, H.C. Introduction to Chemical Engineering Thermodynamics, 3rd ed., McGraw-Hill, NY, 1975. Washburn, E.W., ed., International critical Tables, Vole 4 , MCGraw-Hill, NY, 1928.

Worley, R.P. Chem, Soc, Iondon, 1914, 105, 260-273. 
Chapter 3 Regeneration of Activated carbons

\subsection{Conl: Jatablish a Norxing Definition of Irreversible Adeorption}

The comparisons of adsorption isotherms in chapter 2 provide strong evidence for chemical reaction of phenolic compounds on F-100 activated carbon. Such comparisons do not quantify the amount of reacted sorbate, however, nor do they determine how much of the sorbate can be recovered. In order to study irreversibilities in the adsorption process, an operational definition of the term "irreversible" is needed.

since an activated carbon surface is heterogeneous, adsorbed molecules may have widely different energies of interaction with the surface. A quantitative measure is needed to determine how many of these molecules cannot be desorbed without compromising the integrity of the sorbate molecule or of the surface. The measure should reflect primarily the conditions of the adsorption rather than the desorption process. 


\subsection{Alternative Methods of Regeneration}

Regeneration is most often accomplished by raising the temperature of activated carbon. Thermal regeneration in comercial applications usually involves the following steps; 1) drying of the wet, spent carbon at a temperature slightly above $100^{\circ} \mathrm{C}, 2$ ) heating the carbon to $800^{\circ} \mathrm{C}$ or higher, and 3) gasification of residual organic matter and oxidation of the carbon with oxidizing gases such as steam or carbon dioxide. This process is widely used because it can completely renew the specific area and adsorptive capacity of a carbon surface while less extreme methods may fail to do so. High energy costs and large carbon losses (5-15\%) are common, however, so improved regeneration schemes are sought.

Thermal Gravimetric Analysis, as described in Chapter 1, is a laboratory technique used to study the desorption of organics during a heating step. This technique has been used to differentiate qualitatively between physically and chemically adsorbed phenol (seewald et al., 1983; Magne and Walker, 1986) but does not meet all of the requirements of a regeneration method delineated in the previous section. one objection is that the preliminary drying step and the high temperatures involved may be expected to increase the rates of surface reactions. Another difficulty comes in quantifying the fractions of reversibly and irreversibly adsorbed material. Volatilization of strongly held, but 
reversibly sorbed compound may occur at the same temperatures at which chemisorbed compounds decompose. These two events are not distinguished by gravimetric analysis. Finally, the integrity of the surface is not maintained throughout the heating process. Surface functional groups decompose at temperatures well below $800^{\circ} \mathrm{C}$ (see for example 2arif'yanz et al., 1967).

Many other methods of carbon regeneration have been attempted. Among these, low pressure steaming (Austin, 1984), Soxhlet extraction (Buelow et al., 1973 (a and b); Allen et al., 1971; Pahl et al., 1973; and knickmeyer et al., 1973), and wet oxidation of the surface (Knopp et al., 1978) are inappropriate for this study. The first two procedures elevate the temperature of the carbon, and the later destroys the sorbate and alters the carbon surface. volaeilization of phenol from activated carbon at ambient temperature should not be effective either. Phenol crystals held at ambient temperature under 25 in Hg vacuum for ten days retained their weight (to within 2t). Further, it can be inferred from the results of Magne and Walker (1986) that all of the adsorbed phenol remained on activated carbon dried at ambient temperature for 1 day. Two methods that could be carried out at ambient or lower temperature, and thereby might provide an appropriate basis 
for a definition of irreversible adsorption, are desorption into a supercritical fluid and regeneration with a liquid mixture or solvent.

Desorption of phenol from activated carbon into supercritical carbon dioxide has been studied by Modell et al. (1980). Fluid temperatures from $55^{\circ} \mathrm{C}$ to $120^{\circ} \mathrm{C}$ were used and a secrease of carbon capacity between the first and second cycles of repeated adsorption from water and desorption into $\mathrm{CO}_{2}$ was observed. The decrease in capacity is consistent with reaction of phenol on the surface, but the data are insufficient to determine whether or not the regeneration process affected the amount of irreversible adsorption. The critical temperature $\left(T_{c}\right)$ is $31.10^{\circ}$, so the regeneration could have been carried out at a lower temperature. Supercritical fluid desorption could also be attempted at lower temperature with ethylene $\left(T_{C}=9.5{ }^{\circ} \mathrm{C}\right.$, $P_{C}=$ critical pressure $=49 \mathrm{~atm}$ ) or with triflouromethane $\left(T_{C}=25.9{ }^{\circ} \mathrm{C}, P_{C}=46 \mathrm{~atm}\right)$. Advantages of supercritical fluids over liquids for regeneration of activated carbon are superior mass transfer properties for rapid desorption, low surface tension allowing penetration into very small pores, and high sensitivity of solubilities to changes in solvent density facilitating recovery of solutes from the fluid after desozption. Disadvantages are the complexity and cost of operation at high pressures. 
3.3 Regeneration with Liquids

Liquid mixtures and pure solvents have several desirable characteristics as regenerants. As mentioned, regeneration can be carried out at ambient or lower temperature so that surface reactions will proceed relatively slowly. Too, the strengths of chemical interactions with phenolics can be manipulated: Regenerants may weakly solvate the desorbed compound or may react reversibly with it (e.g. acid-base reactions). This degree of freedom allows a trade-off between the driving force for desorption and the ease of recovering desorbed material from the solvent. It is also possible to improve the driving force for desorption by introducing a compound that displaces the sorbate fre" the sur*ace. Finally, the amount of desorbed solute is readily guantified and there is the possibility of desorbing reaction products that are not covalently bonded to the surface.

There are also potential problems with liquid regenerants. Diffusivities in a liquid are much lower than in a gas, and the time required for complete desorption into a liquid may allow surface reactions to proceed to a measurable extent. Non-reversible chemical reaction of the regenerant with the surface and/or with sorbed species is possible. Removal of the regenerant from the activated carbon might also be a problem. The experiments reported 
in the remainder of this section indicate that regeneration with liquids can provide an adequate measure of irreversible adsorption despite these potential drawbacks.

\subsubsection{Effectiveness of Various Liquid Regenerants}

The first test of the efectiveness of various regenerants was to load an activated carbon with phenol and then regenerate equal portions of it with equal volumes of several solvents. A 4.2 gram sample of $10 \times 20$ mesh Filtrasorb-100 was immersed in 2.4 liters of an aqueous solution containing $6.38 \times 10^{-3} \mathrm{M}$ phenol. The vessel had a capacity of 4 liters and was not agitated. After 21.3 hours the mixture was vacuum filtered to collect the carbon, and the carbon was weighed. Equal weight $(0.33 \mathrm{~g})$ portions of wet carbon were contacted with $100 \mathrm{ml}$ each of various solvents for 66.3 hours. Phenol concentrations in the aqueous raffinate and in the regenerants were determined by GC, excent for those in the basic aqueous solutions which were determined by HPLC. The first column of Table $3.1 \mathrm{a}$ lists the percentage of the adsorbed phenol removed from the carbon by each regenerant.

The first four solvents listed all removed about the same amount of phenol. Additional desorption time was allowed (for a total of 120 hours) without significant changes in the recoveries, as shown in Table $3.1 \mathrm{~b}$. Additional solvent $(100 \mathrm{ml})$ was added to each of the four 
Table 3.1a Batch Regeneration of Phenol from F-100 Activated Carbon

Percent Removal

\begin{tabular}{|c|c|c|}
\hline Solvent & $\begin{array}{c}F=100 \\
(10 \times 20 \text { mesh })\end{array}$ & $\begin{array}{c}\text { Norit } A^{*} \\
\text { (Powdered) }\end{array}$ \\
\hline Metyl isobutyl ketone & 88.9 & $N D^{*}$ \\
\hline Acetone & 88.8 & 85.7 \\
\hline Dimethylformamide & 88.6 & $\$ 5.0$ \\
\hline Diisopropylether & 88.4 & ND \\
\hline Ethanol & 85.3 & 88.3 \\
\hline Benzene & 83.1 & 76.5 \\
\hline Ethyl Acetate & 82.9 & 76.3 \\
\hline Tolvene & 79.1 & 82.5 \\
\hline Methanol & 78.1 & 70.6 \\
\hline 2-propanol & 78.0 & 73.7 \\
\hline O.IN Sodium hydroxide & ND & ND \\
\hline $0.1 N$ Ammonium hydroxide & 30.9 & ND \\
\hline Distilled Water & 16.1 & 18.3 \\
\hline Contact Time (or Adsorption (hours) & 21.3 & 0.5 \\
\hline Contuct Time for Desorption (hours) & 66.3 & 0.5 \\
\hline Carbon Loading (mg/gdc) & 68.9 & 200.0 \\
\hline Solvent to Carbon Ratio (ml/gdc) & 473.0 & 10.0 \\
\hline
\end{tabular}

'From Cooney et al., 1983.

- ND $=$ Not Determined

Table $3.1 \mathrm{~b}$ Completeness of Batch Regeneration of Phenol From F-100

\begin{tabular}{|c|c|c|c|c|}
\hline \multirow[b]{2}{*}{ Solvent } & \multicolumn{4}{|c|}{ Percent Removal } \\
\hline & $\begin{array}{c}\text { For } \\
\text { Base } \\
\text { Case }\end{array}$ & $\begin{array}{c}\text { With } \\
\text { Additional } \\
\text { Desorption Time } \\
\text { (Total }=120 \mathrm{~h} \text { ) }\end{array}$ & $\begin{array}{c}\text { With } \\
\text { Additional } \\
\text { Solvent } \\
\text { (Total= } \\
946 \mathrm{ml} / \mathrm{gdc} \text { ) }\end{array}$ & $\begin{array}{c}\text { With } \\
\text {. dditional } \\
\text { Batch } \\
\text { (473 ml } / 8 d c)\end{array}$ \\
\hline Metyl isobutyl ketone & 88.9 & 88.8 & 88.9 & 88.9 \\
\hline Acetone & 88.8 & 88.8 & 88.9 & 88.9 \\
\hline n,n-Dimethylformamide & 88.6 & 88.6 & 88.7 & 88.7 \\
\hline Diisopropyl ether & 88.4 & 88.6 & 88.6 & 88.6 \\
\hline
\end{tabular}

'Conditions for the base case are given in Table 3.19 
regenerant mixtures, and the recoveries improved only slightly after 24 hours, the changes in recoveries being within experimental error. The carbon was separated from each of the four regenerant mixtures by vacuum filtration and $100 \mathrm{ml}$ of fresh solvent was added. Phonol was detected in trace, but not measurable, quantities after 48 hours. These results suggest that each of the four solvents recovered virtually all of the reversibly sorbed phenol. The different chemical natures of the solvents were not important at the high solvent to carbon ratios employed. As long as a high enough solvent to carbon ratio is used, therefore, a single regeneration with one of the top four solvents can be expected to remove virtually all of the reversibly sorbed phenol.

The remaining organic solvents listed in Table $3.1 \mathrm{a}$ varied in their ability to remove phenol from the carbon, but the differences were not great. Aromatics, alcohols, and ethyl acetate recovered between 87 and 97 percent of the amount recovered by methyl isobutyl ketone (MIBK). These results may be compared to those from an earlier study summarized in the second column of Table 3.1 a (Cooney et al., 1983). The variation in recoveries was somewhat greater in the earlia: study, which reflects the much lower solvent to carbon ratio and the higher loading. The relative ordering of solvents is not identical, but, in general, there are more similarities than differences. 
Most importantly, dimethyl formamide, ethanol, and acetone gave greater removals than did other solvents tested in both studies. It should be noted t'rat in the study of Cooney et al., a marked improvement in the recovery of phenol with acetone (to 90.27 ) was achieved by doubling the solvent to carbon ratio.

Phenol recoveries with water and with the basic aqueous solutions were less than those with organic regenerants. The strongly polar nature of water favors the partioning of phenol to the solid-liguid interface. Also, the high surface tension of water favors partitioning of phenol to the liquid-solid interface because greater reductions in interfacial energy result per mole of phenol accumulating. The low percent removal for distilled water reflects the fact that phenol-water-F-100 adsorption isotherms are steep in the dilute region and indicates that large quantities of water would be required to desorb the reversibly bound phenol.

Addition of base enhances the recovery since much of the phenol is then in the form of phenolate ion, which is well solvated by water and is expected to have relatively little affinity for the surface a- high pH (Mattson et al., 1969; Müller et al., 1980; and see section 5.2.4). Ammonium hydroxide was much less effective than sodium hydroxide, presumably due to the relative strengths of the bases. Basic aqueous solutions promote oxidation of 
phenolics (Penketh et al., 1957: Lim et al., 1983: Chin et al., 1985; and see section 5.2.4), and the regenerant mixtures did develop a yellow coloration. oxidation during the desorption process linits the recovery of phenol and could interfere with the identification of reaction products formed in the adsorption process. Incomplete recovery of phenol from activated carbon by Goto et al. (1986, see Chapter 1) may have been due to oxidation.

Previous studies have attempted to rationalize the differing abilities of solvents to regenerate phenol from activated carbon (Pahl et al., 1973; Knickmeyer et al., 1973; and Fuchs and Kuhn, 2976). In the first two studies, the solvents were held at $40^{\circ} \mathrm{C}$, so surface reactions might have affected the results. The highest recovery achieved in either of the first two studies was $70 \%$, and the recoveries were generally much lower. Despite the elevated temperature, the relative effectivenesses of the solvents were consistent with the ordering in Table 3.1a. From best to worst, the solvents were: dimethylformamide, benzene, tetrahydrofuran, dioxane, methanol, acetonitrile, dodecyl alcohol, cellulose acetate, cyclohexane, and water. Attempts to correlate reaeneration ability with solubility parameters, molar volumes, dielectric constants, dipole moments, and $\mathrm{pK}_{\mathrm{a}}$ values were unsuccessful. Limited success was achieved in relating heats of solution of phenol in various solvents, and measured heats of hydrogen bond 
formation for phenol with solvent molecules, to desorption efficiencies. This success, while qualitative, suggests that specific interactions between phenol and solvent molecules play an important role at the (lower) solvent to carbon ratios employed in these studies.

A quantitative scale of adduct interaction energies has been developed by Drago (2973, and Drago et al., 1971). The method correlates enthalpies of adduct formation $(A H)$ in the gas phase and in poorly solvating solvents with electron donor and acceptor properties. Donor-acceptor properties are resolved into electrostatic and covalent contributions, and two parameters $E$ and $C$ are thereby assigned to each compound. The governing equation is:

$$
\Delta H=E_{A} E_{2}+C_{A} C_{1}
$$

where the subscripts $A$ and $B$ indicate the acceptor and the donor respectively. The original set of $E$ and $C$ parameters was determined mainly from enthalpy data for interactions of iodine and of phenol, so this method should be very useful for gauging solvent-phenol interactions. Table 3.2 shows results of Drago's work that are relevant to the experiment being discussed.

According to the enthalpies of interaction given in Table 3.2, dimethylformamide (DMF) ant dissopropylether (DIPE) should be strong regenerants o: imilar efficacy. 
Table 3.2

Donor-Acceptor Interaction Parameters from the Model of Drago*

\begin{tabular}{llll} 
Acceptor & $C_{\mathrm{A}}$ & $\mathbf{E}_{\mathrm{A}}$ \\
\hline Phenol & 0.442 & 4.33 \\
Donors & $\mathrm{C}_{\mathrm{B}}$ & $\mathrm{E}_{\mathrm{B}}$ & $-\Delta \mathrm{H}=\mathrm{E}_{\mathrm{A}} \mathrm{E}_{\mathrm{B}}+\mathrm{C}_{\mathrm{A}} \mathrm{C}_{\mathrm{B}} \ldots$ \\
\hline Dimethylformamide & 2.48 & 1.23 & 6.42 \\
Diisopropylether & 3.19 & 1.11 & 6.22 \\
Acetone & 2.33 & 0.987 & 5.30 \\
Ethyl Acetate & 1.74 & 0.975 & 4.99 \\
Benzene & 2.42 & 0.486 & 2.42 \\
\hline
\end{tabular}

'Drago et al., 1971 and Drago, 1973.

"Enthalpy units are keal/mol; E and $C$ parameter units are (kcal/mole) $1 / 2$. 
The results in Table $3.1 \mathrm{a}$ support that prediction. Acetone is expected to be less effective, and this was true for the regeneration of Norit $A$, but not for regeneration of $F-100$ at the very high solvent to carbon ratio. Ethyl acetate should be less effective than DMF, DIPE, and acetone and was in both experiments. Finally, benzene has a predicted interaction enthalpy well below those of the other four compounds in Table 3.2 , but was not far worse as a regenerant. These results reveal that interactions other than those of the solvent and phenol must be considered. The presence of water (a strong electron donor and electron acceptor) has been shown to decrease the regeneration effectiveness of solvents markedly (Pahl et al., 1973; and Knickmeyer et al., 1973). The surface properties of the activated carbon should also be important.

The experimental results in Table $3.1 \mathrm{a}$ and Table $3.1 \mathrm{~b}$ strongly suggest that all of the reversibly sorbed phenol on F-100 could be desorbed with a high ratio of acetone volume to carbon weight. Acetone has additional virtues as a regenerant, including high volatility and complete miscibility with water. These facts are important because, in most commercial processes, residual solvent would be removed from the carbon following desorption. Flushing with water or steam would be economical methods to remove acetone from activated carbon. Miscibility with water was particularly important for this study because it was 
desired to remove residual solvent from the carbon without elevating the temperature. Acetone also has the virtues of being relatively innocuous and of being less expensive than MIBK, DMF, and DIPE. Consequently, acetone was chosen as an appropriate regeneration solvent for further studies of irreversible adsorption.

\subsubsection{Effectivenese of Phenolie Displacers}

Additional driving force for removing reversibly sorbed phenolic compounds from activated carbons can be provided through a 'displacer'. A displacer is "a strongly adsorbed dominant adsorbate" (Thakkar and Manes, 1987). An effective displacer will have more affinity for the surface and/or will be present in much greater quanity in the regenerant than the original sorbate.

Thakkar and Manes found that phenol, 2-nitrophenol, and 4-chloro-3-methylphenol could be quantitatively displaced from Filtrasorb-400 activated carbon. The phenolic compounds were adsorbed from a solution of multiple solutes in methylene chloride over a contact time of at least 48 hours. Very low loadings resulted (of order 1 milligram per gram of dry carbon, $1 \mathrm{mg} / \mathrm{gdc}$ ), and it was expected that the loading of any one compound was independent of the loadings of the other compounds. After the adsorption contact, the methylene chloride was evaporated, and the 'dry' carbon was contacted with a mixture of $80 \mathrm{~g} / \mathrm{L}$ 
phenolic displacer in methanol. Phenol was the displacer for 4-chloro-3-methylphenol and for 2-nitrophenol, and 4-chloro-3-methylphenol was the displacer for phenol. The investigators concluded that none of the phenolic compounds tested reacted with the carbon.

In the present work, tests were run to see whether mixtures containing phenolic displacers could desorb more phenol than could be removed by pure solvents, when high solvent to carbon ratios and multiple batch contacts were used. For adsorption, $20 \times 40$ mesh Witco Columbia activated carbon was contacted with a dilute solution of phenol in water under various conditions, as summarized in Table 3.3 . The carbon was separated from solution by vacuum filtration and weighed to determine the ratio of wet to dry carbon. Regeneration of equal-weight portions of carbon was accomplished through batch contacts continued until phenol could not be detected in a regenerant after at least 2 days.

The results corresponding to trial number 1 in Table 3.3 show that phenol was completely recovered with methanol and with 4-chloro-3-methyl-phenol in methanol $(80 \mathrm{~g} / \mathrm{L})$ after an adsorption contact for 24 hours at ambient temperature. The percent desorption cannot be greater than 100, and the higher value reported for desorption into methanol reflects experimental inaccuracies. After the final desorption contact with methanol, the carbon was vacuum filtered and 
Table 3.3 Desorption of Phenol from Witco Columbia Activated Carbon into Solvents and with Displacers

Trial Number

123

Adsorption"

Temperature $(\circ \mathrm{C})$

Ambient

80

80

Contact Time (hours)

24

120

120

Loading ( $\mathrm{mole} / \mathrm{m}^{2}$ )

1.57

3.49

3.37

Desorotion"

\% Desorption into MeOH

104.1

34.9

35.2

Additional \% Desorption into 4C3MP in MeOH

0

0

Additional \% Desorption into TCP in MeOH

$\%$ Desorption into 4 C3MP in $\mathrm{MeOH}$

99.8

34.2

$\%$ Desorption into $3 N P$ in

$\mathrm{MeOH}$

\% Desorption into TCP in

$\mathrm{MeOH}$

$-$

34.9

\% Desorption into Acetone

-

35.4

\% Desorption into TCP in Acetone

"For trial 1,600 ml of $6.38 \times 10-3 \mathrm{M}$ phenol in water and $1.05 \mathrm{~g}$ dry carbon were contacted. For trials 2 and $3,600 \mathrm{ml}$ of $1.28 \times 10^{-2} \mathrm{M}$ phenol in water and $1.05 \mathrm{~g}$ dry carbon were contacted. Contact was in a I liter glass vessel at ambient temperature and with no agitation.

"Wet carbon samples of weight equivalent to $0.2 \mathrm{~g}$ dry carbon were contacted with regenerants for at least 48 hours in well agitated vessels at ambient temperature. Multiple batch desorptions were carried out until phenol could not be deteeted in the regenerant.

Abbreviations: $\quad \mathrm{MeOH}=$ Methanol

4C3MP = 4-Chloro-3-methylphenol

TCP $=2,4,6-$ Trichlorophenol

$3 N P=3-N i t r o p h e n o l$ 
contacted with $80 \mathrm{~g} / \mathrm{L}$ 4-chloro-3-methylphenol in methanol. No phenol was detected in the mixture after 48 hours. These results indicate that all of the phenol was reversibly adsorbed and that methanol was as effective as the solution containing displacer for recovering reversibly sorbed solute. It also appears that witco Columbia has less tendency to promote irreversible adsorption than does Filtrasorb-100, at least for the adsorption conditions of Trial 1 (see section 3.3 .1 for comparison).

A higher adsorption temperature and a longer adsorption contact time were used in the second and third trials in order to promote irreversible adsorption. Correspondingly, desorption into methanol was incomplete. In the second trial, regeneration with methanol was followed by a contact with 4-chloro-3-methylphenol in methanol, but no further desorption occurred. In the third trial, regeneration with methanol was followed by a contact with $2,4,6$-trichlorophenol in methanol, with no further desorption. In addition, four mixtures of phenolic displacers in a solvent (each $80 \mathrm{~g}$ displacer per liter of solution) were contacted with fully loaded carbon and, within experimental uncertainty, each recovered the same quantity of phenol as did methanol alone. In the third trial, acetone also recovered the same amount of phenol as did methanol. These results indicate that solvents 
containing displacers are no more effective for desorbing phenol than are pure solvents, when there is no restriction on the quantity of regenerant used.

\subsection{References}

Allen, S.C.; Pahl, R.H.; and Mayhan, K.G. Water Res., 1971, 5. $13-18$.

Alstin, G.T. Shreve's Chemical Brogess Industrien, MCGraw-Hil1, NY, 137-138, 1984.

Buelow, R.พ.: Carswell, J.K.: and symons, J.M. I, Ame hater Works Assoc, $1373(\mathrm{a}), 65(1), 57-72$.

Buelow, R.W.: Carswell, J.K.; and symons, J.M. I, Am, water Works Assoc., $1973(b), 65(3), 195-199$.

Chin, K.C.; Cha, J.A.: Lim, P.K. Ind, Eng, Chem, Process Des, Dev, 1985, 24, 339-343.

Cooney, D.O.; Nagerl, A.: and Hines, A.L. Water Res., 1983, 17. $403-410$.

Drago, R.S. Structure and Bonding (Barlinl, 1973, 15, 73-139.

Drago, R.S.: Vogel, G.C.; and Needham, T.E. I. Am. Chem. Sec., 1971, 23, 6014-6026.

Fuchs, F. and Kuhn, W. "The Use of Activated Carbon to Analyze Natural Waters with Regard to Their Behavior in 
Waterworks Filters", Translation of Reports on special Problems of water Technology, Volume 9-Adsorption, EPA 600/9-76-030, 1976.

knickmeyer, W.W.: Mayhan, K.G.; and Bertrand, G.L. Water Res., 1973, Z, 1323-1330.

Knopp, P.V.; Gitchel, w.B.: Meidl, J.A.; and Berndt C.L. "Wet Oxidation Regeneration" Carbon Adsorgtion Handbook, Eds. Cheremisinoff, P.N., and Ellerbusch, F., Ann Arbor Science Publishers, Aתn Arbor, MI, 1978.

Lim, P.K.: Cha, J.A.; and Patel, C.P. Ind, Eng, Chem. Process Des, Dev, 1983, 22, 477-482.

Magne, P. and Walker, P.L. Carben, 1986, 24, 101-107. Mattson, J.S.; Mark, H.B.: Malbin, M.D.; Weber, W.J.: and Crittenden, J.C. I, colloid Interface Sci,, 1969, 31, 116-130.

Modell, M.; deFilippi, R.; and Krukonis, V., "Regeneration of Activated Carbon with Supercritical Carbon Dioxide", Activated carton Adsorption of organics from the Aoueous Phase, Volume 1, Eds. Suffet, I.H. and McGuire, M.J., Ann Arbor Science Publishers, Inc., Ann Arbor, MI, 1980.

Müller, G.; Radke, C.J.; and Prausnitz, J.M. I. Phys. chen., 1980, 84, 369-376.

Pahl, R.H.; Mayhan, K.G.; and Bertrand, G.L. Water Res., $1973, I, 1309-1322$. 
Penketh, G.E. J. Apol, Chem, 1957, I, 512-521.

Seewald, H.; Klein, J.; and Juntgen, H., "Correlation Between Desorption Behavior and Adsorption Characteristics of Organic Substances on Activated Carbon". Fundamentals of Adsorption: Procendings of the Engineering Eoundation Conference held at Schlore Elnov, Bavaria, West Gemany, May 6-11, 1983, ads. Mayers, A.L., and Belfort, G., Engineering Foundation, New York, 557-565, 1983.

Thakkar, S. and Manes, M. Enviren. Sci. Technol., 1987, 21, 546-549.

Zarif'yanz, Y.A.: Kiselev, V.F.: Lezhnev, N.N.; and Nikitina, D.v. Carbon, 1967, 5, 127-135. 
Chapter \& Characterizing the Reaction

4.1 Iufluences of Adsorbent Properties on Irreversible Adsorption

Figures 2.1 and $2.2 \mathrm{a}$ showed that adsorbent properties influence the surface excesses of phenolic compounds. It has also been established that chemical changes may take place at an interface resulting in irreversible adsorptior. The characteristics of a solid that promote irreversible adsorption remain to be determined. This section explores the connection between adsorbent properties and the relative tendencies of the adsorbents to promote irreversible adsorption. Accordingly, a wide variety of adsorbents are tested.

\subsection{1 xaD-4, A Reveraibly Adsorbing Resin}

Macroreticular synthetic resins without ion exchange functional groups are effective for removing phenolic compounds from water (see e.g. Fox, 1978; van rliet and Weber, 1981; Chriswell et al., 1977; and Costa and Rodrigues, 1982). These resins have high surface areas (usually 300-800 $\mathrm{m}^{2} / 9$ ) because each particle is an agglomeration of microspheres with diameters as small as $10^{-4} \mathrm{~mm}$. The resins should interact with sorbed organics 
through dispersion forces, and binding energies should generally be lower than those for the same sorbates on an activated carbr:s (Gustafson et al., 1968). Under these conditions, adsorption should be completely reversible through solvent regeneration. Experiments were conducted to test this hypothesis for XAD-4, a macroreticular adsorbent with minimal surface funetionalityl.

Amberlite XAD-4 is a polystyrene-divinylbenzene copolymer with a very high surface area $1725 \mathrm{~m}^{2} / 9$, Tabla 2.1b). This copolymer has an aromatic structure and is nonpolar and hydrophobic. These characteristics favor the sorption of phenolics from water and account for larger surface excesses on XAD-4 than at a liquid-vapor interface (see Figure 2.2a). The enthalpies of binding for m-chlorophenol and 2,4-dichlorophenol on this sorbent are -4 and $-6 \mathrm{Kcal} / \mathrm{mc}$ respectively (Paleos, 1969), indicating that these compounds aze physisorbed. Accordingly, complete recovery of phenol, adsorbed from an acidic aqueous stream at ambient tomperature, has been achieved through regeneration with acetone and with methanol (Crook et al., 1975).

Complete recovery of phenol and resorcinol sorbed from neutral aqueous solutions at $25^{\circ} \mathrm{C}$ was achieved in this work for the batch adsorptions reported in Figure 2.2a. The

1 XAD-4 contains no heteroatoms, but contains pendant vinyl groups that may be reactive (see Bootsma et al. 1984). 
loaded resin was regenerated with acetone according to the method detailed in Section 4.1.4.1. All mass balances closed to within 5\%, as shown in Figure 4.1 . In Figure 4.1, the open symbols represent total uptakes while the solid bars represent regeneration amounts. symbols at the same solute mole fraction correspond to the same trial.

The rate of chemisorption of phenol on activated carbon increases with increasing temperature. Additional tests were conducted to determine whether XAD-4 promotes irreversible adsorption at elevated temperature. The results $6:$ adsorption contacts at $80^{\circ} \mathrm{C}$ were reported in Figure 2.7 and are shown again as total uptakes in Figure 4.2. Also shown in Figure 4.2 are reversible uptakes. Regeneration with acetone was complete with all mass balances closing to within $5 \%$.

The regeneration results show that XAD-4 dues not promote irreversible adsorption under the conditions tested. This fact implies that activated carbon has specific features that cause irreversibilities. concentration and reaction of phenolic molecules at an interface that does not participate in the reactions, or chemical reactions in the bulk solution, cannot account for non-closure of mass balances for carbons. similarly, biodegradation cannot be occurring unless the bacteria are introduced with the carbon, and the drying process should 


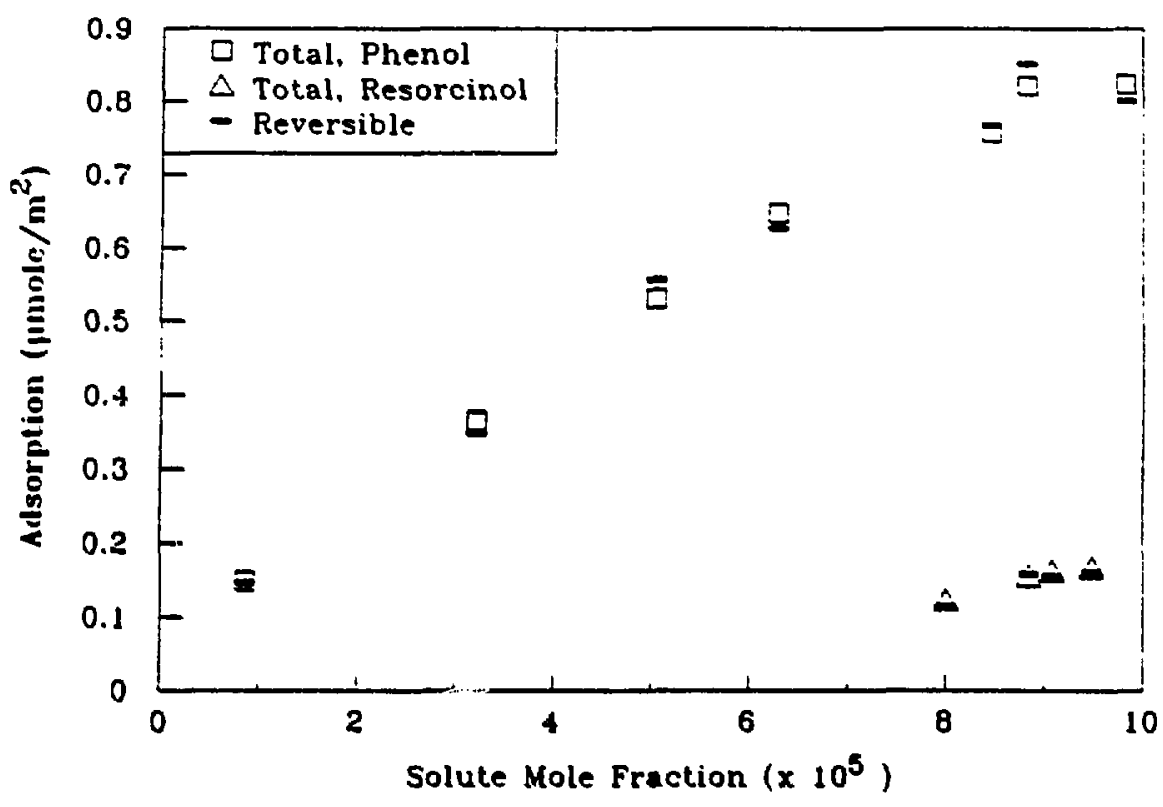

Figure 4.1 Total and Reversilsle Adsorption of Phenol and Resorcinol from Water onto XAD-4. Adsorption Contacts were at $25 \cdot \mathrm{C}$. 


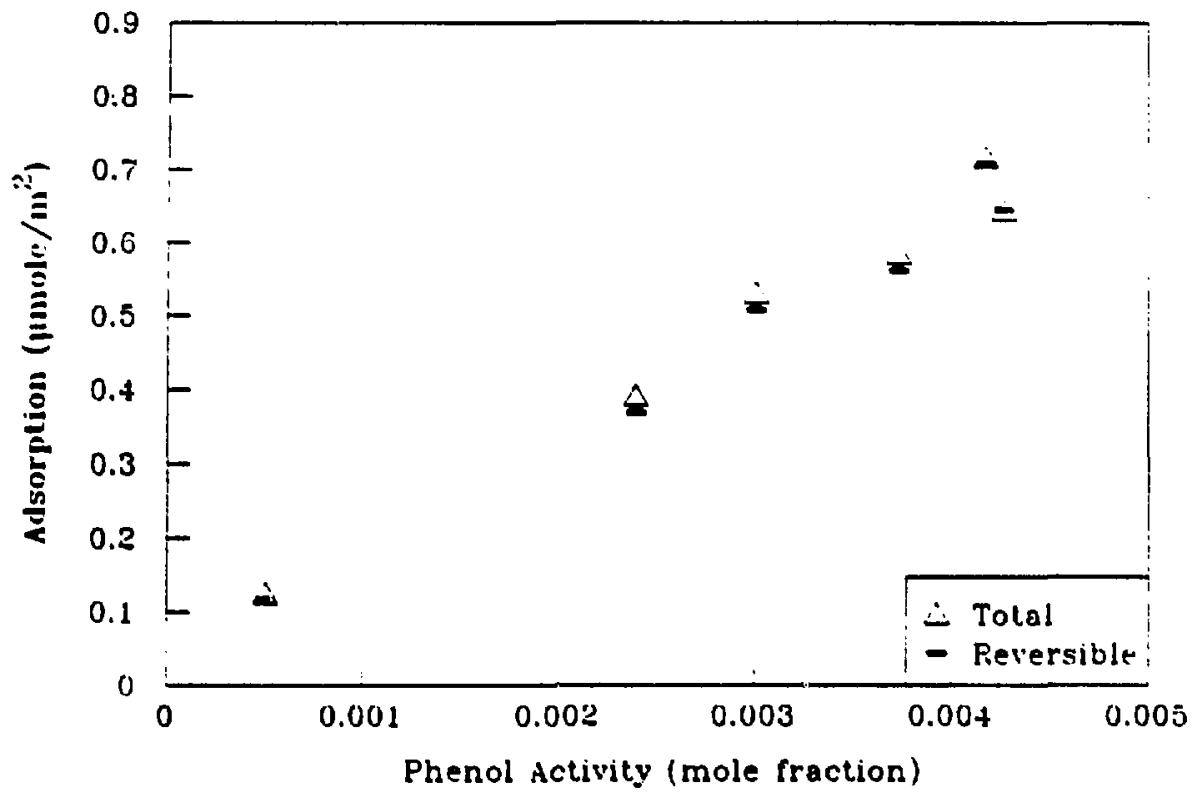

Figure 4.2 Total and Reversible Adsorption of Phenol from Water onto XAD-4. Adsorption Contacts were at $90^{\circ} \mathrm{C}$. 
prevent this. Therefore, it is important to examine the surface properties of activated carbon and their influences on irreversible adsorption.

\subsection{2 surface Properties of Carbons That Affect rotal Optakes of Phenolic Compounds}

Several investigations have shown that surface properties of carbon influence the adsorption densities of phenolic compounds, but almost none of these studies address the question of reversibility. It is reasonable to suspect, however, that the properties which influence total uptakes may influence irreversible uptakes as well. This section reviews some of the studies most relevant to this topic.

Activated carbon consists of microcrystallites which, in turn, consist of disrupted graphitic layers (Coughlin and Tan, 1968). A graphitic layer may be regarded as a large polynuclear aromatic molecule that interacts with phenolic sorbates through dispersion forces. Free valences at the edges of graphitic layers may react with heteroatoms to form functional groups, or they may remain as reactive sites. Functional groups that have been identified or inferred to be on ca-bons include carboxylic acids, lactones, carbonyls, phenols, and sulphonyls ${ }^{2}$. These groups can interact with phenolic sorbates through Lewis mentioned are thought to be the most common. 
acid-Lewis base associations. Finally, activated carbons generally contain inorganic materials in the form of ash. Metallic elements such as iron are common and, at least potentially, could catalyze sorbate reactions.

Coughlin and Ezra (1968), Coughlin and $\operatorname{Tan}$ (1968), and Coughlin et al. (1968) found that isotherms for phenol adsorbed from water onto various carbons had two plateaus, one at low concentrations $(0.1-0.3 \mathrm{mmoles} / 1)$ and one at higher concentrations $(100-300 \mathrm{mmoles} / 1)^{3}$. Acidic surface oxides fformed through wet oxidation with ammonium persulfate and thought to b. primarily in the form of carboxyls and lactonec (Cookson, 1978)] reduced uptakes per unit area in the dilute region, but usually had little effect on adsorption from more concentrated solutions. The investigators attributed decreased uptakes from dilute solutions to weakened dispersive interactions between pi electrons in carbons and the pi electrons of phenol. outgassing oxidized carbons at $890^{\circ} \mathrm{C}$ removed the surface oxides and restored the original adsorption characteristics.

Epstein et 21. (1971) studied adsorption of p-nitrophenol onto pyrolytic carbon in oxidized and reduced forms. Adsorption was favored on the oxidized

3 Dual plateaus of this type were not observed in this work. 
carbon, which was thought to possess primarily quinone groups. The reduced carbon was thought to possess mostly hydroguinone groups.

Eptein's findings support the contention of Mattson et al. (1969) that donor-acceptor complexes are the primary form in which phenolic compounds are adsorbed onto many activated carbons. Carbonyl oxygen on a carbon donates electrons, while the aromatic rings of sorbates accept electrons. Mattson et al. also found that nitrophenols and nitrobenzene interacted more strongly with a carbon than did phenol, and concluded that this was due to the ability of nitro-groups to withdraw electrons from aromatic rings. In addition, they suggested that oxidation of carbon as carried out by Coughlin and coworkers decreased phenol adsorption because carbonyl groups were oxidized.

Mahajan et al. (1980) disagree with Mattson et al. Mahajan and coworkers found no difference in phenol uptakes for carbons with different surface concentrations of carbonyl groups. These investigators confirmed the observation that acidic surface oxides decrease phenol uptakes from dilute solutions and suggested that preferential uptake of water is responsible. They argued that clusters of water molecules could form on oxygenated sites and couid potentially block pore entrances. This view is supported by increased phenol uptakes onto oxidized 
carbon from a nonpolar solvent (cyclohexane) and decreased uptakes onto a carbon with carboxyl hydrogens exchanged for sodium atoms.

Mahajan et al. also found decreased phenol uptakes on carbons with reduced ash contents. They surmised that this could be due to electron donating abilities of impurities and/or the abilities of alkali and alkaline earth impurities to increase the basicity of a carbon surface.

In summary, the chemical properties of activated carbons markedly influence the uptake of phenolic compounds from water. Acidic surface oxides hinder adsorption, while the influences of basic surface oxitus are open to debate. Ash has been shown to promote phenol adsorption, but the reasons for this are speculative. Activated carbons contain unpaired electrons, but no studies could be located that demonstrate a link between unpaired electrons and phenol uptakes.

\subsubsection{Charasterisation of Activated Carbon surface Crisuistries}

Given the discussion in the previous section, chemical characterization of carbon is important to the understanding of ddsorption interactions. Unfortunately, activated carbon is difficult to characterize, and most properties of interest must be inferred. Three experimental methods were used to gain information about 
carbons used in this work -- elemental analysis, reaction with lithium aluminum hydride, and infrared spectroscopy. The techniques and results for vendor-supplied carbons are summarized in this section.

\subsubsection{Elemental Analyais}

Elemental analysis of adsorbents was performed by Mr. v. Tashinian of the College of Chemistry Microanalytical Laboratory at the University of California, Berkeley. weight fractions of carbon, hydrogen, and nitrogen were determined with a Perkin-Elmer Model 240 Elemental Analyser (Perkin-Elmer, Norwalk, Conneticut). Samples were burned in excess oxygen, and the combustion products $\mathrm{CO}_{2}, \mathrm{H}_{2}$, and $\mathrm{N}_{2}$ were quantitatively determined with a thermal conductivity detector.

The weight fraction of sulfur in each adsorbent was established with the Grote Combustion method. Each sample was burned in excess oxygen, and the combustion products were contacted with a solution of $3 t$ hydrogen peroxide in water. This solution absorbed sulfoxides which precipitate as $\mathrm{BaSO}_{4}$ after addition $4 \mathrm{BaCl}_{2}$. The $\mathrm{BaSO}_{4}$ was weighed, and the amount of sulfur in the original sample was determined by mass balance.

The ash content of activated carbons was determined from the weight of the residue remaining after the Grote Combustion analysis. Weight percents of ash components ( $K$, 
$\mathrm{Mn}, \mathrm{Na}, \mathrm{Cu}, \mathrm{Co}, \mathrm{Fe}$, and $\mathrm{Cr}$ ) were determined by disolving a carbon sample in a mixture of concentrated acids ( 1 part $\mathrm{H}_{2} \mathrm{SO}_{4}$ to 1 part $\mathrm{HNO}_{3}$ by volume), diluting the mixture with water, and then measuzing the atomic absorption of individual elements. A Perkin Elmer 2380 Atomic Absorption Spectrophotometer was used for the analyses.

The weight fraction of oxygen in an adsorbent was measured by pyrolyzing a sample in helium at $970^{\circ} \mathrm{C}$. The oxygen formed carbon monoxide, which was separated from acid gases by passing the combustion products through a mixture of sodium hydroxide on asbestos. The carbon monoxide passed through the mixture and was converted to $\mathrm{CO}_{2}$ over a mixture of $\mathrm{Cu}$ and $\mathrm{Cuo}$ at $670^{\circ} \mathrm{C}$. The amount of carbon dioxide formed was determined with a thermal conductivity detector, and the amount of oxygen in the original sample was back calculated. This procedure does not measure oxygen present as stable oxides in the ash but does measure oxygen in adsorbed water.

Elemental analyses of vendor-supplied activated carbons are presented in Table 4.1. The analyses for the first three carbons listed were reported in an earlier study from this laboratory (Munson, 1985). Due to equipment difficulties, no oxygen analyses were available for pretreated samples of Filtrasorb-100 and witco Columbia. oxygen analyses were available for samples of these carbons that were rinsed with water, but not subjected to soxhlet 
Table 4.1 Elemental Analysis of Vendor-Supplied Activated Carbons

$$
\text { Elemental Analyses (Weight Percent) }
$$

Activated

\begin{tabular}{lccccccc} 
Carbon & $\mathrm{C}$ & $\mathrm{H}$ & $\mathrm{N}$ & $\mathrm{S}$ & $\mathrm{O}$ & Ash & Total \\
\hline Witcarb 950 & 95.14 & 0.55 & 0.06 & 2.73 & 0.90 & 0.5 & 99.9 \\
Super Sorb & 79.91 & 0.45 & 0.10 & 0.11 & 0.76 & 19.0 & 100.3 \\
Row 0.8s & 77.22 & 0.82 & 0.62 & 0.14 & 10.28 & 4.6 & 93.7 \\
F-100 & 88.95 & 0.05 & 0.41 & 0.78 & $(3.41)^{*}$ & 6.4 & 96.6 \\
Columbia & 93.70 & 0.18 & 0.00 & 3.31 & $(2.41)^{*}$ & 0.40 & 97.6 \\
\hline
\end{tabular}

- Determined by difference and not included in the toral. 
extraction in the manner described in section 2.1. A sample of F-100 contained 2.16 weight percent oxygen, while a sample of Witco Columbia contained 2.12 weight percent oxygen. These values are reasonable for the pretreated samples as well, given the amounts of nonclosure for the mass balancas.

It should be emphasized that the elemental analyses measure bulk rather than surface properties. These activated carbons have extremely high specific surface areas, however, so high percentages of the elements are located at the surfaces. Ths percentage of oxygen at the surface should be especially high when the raw material for the activated carbon contains little oxygen (i.e. petroleum or coal, but not peat).

\subsubsection{Metive Bydrogen}

Hydrogen atoms that react with lithium aluminum hydride in ether to evolve hydrogen gas are defined as active hydrogen. In general, hydrogen atoms bonded to nitrogen, oxygen, or sulfur atoms are active. LiAlH, is a reducing agent and acts $\mathrm{as}$ an $\mathrm{H}^{-}$donor. One mole of $\mathrm{H}_{2}(\mathrm{~g})$ is liberated and one quarter mole of hydride consumed for each mole of active hydrogen. $\mathrm{LiAlH}_{4}$ does not reduce ethers, ketals, acetals or carbon-carbon double or triple bonds. Alcohol hydrogens are active, but the group is not reduced. 
Figure 4.3 illustrates reactions expected for selected functional groups commonly present on activated carbons. The carboxylic acid hydrogen is active, and the group is reduced to a dissociated alcohol. The carbonyl group contains no hydrogen, but is reduced by the hydride. The phenolic hydrogen is active, but the group is not reduced. If any of the reacted groups is contacted with a protic solvent: (e.g. water), an alcohol forms. While active hydrogen atoms are not always acidic in the Brönsted sense, the amount of $\mathrm{H}_{2}(\mathrm{~g})$ evolved during raduction of a carbon reflects the surface acidity. Riven (1963) found good agreement between the active hydrogen contents of carbon blacks and their acid contents as determined by titration with NaOH.

The experimental method used in this work was adapted from that used by Riven for carbon blacks. A mixture of 5 grams of $\mathrm{LiAlH}_{4}$ and $100 \mathrm{ml}$ of spectrograde diglyme [bis(2-methoxyethylether)] was prepared under a nitrogen atmosphere. A known weight of dry adsorbent for chemical to be tested) and a magnetic stir bar were placed in a 25 ml, three-neck flask, and the flask was sealed. One neck of the flask was sealed with a ground glass stopper. A second neck was seale: with a rubber serum stopper attached to a teflon adapter. The third leg of the flask was connected via tygon tubing to one leg of a manometer filled with heptane. The sealed flask was placed in a bath of 


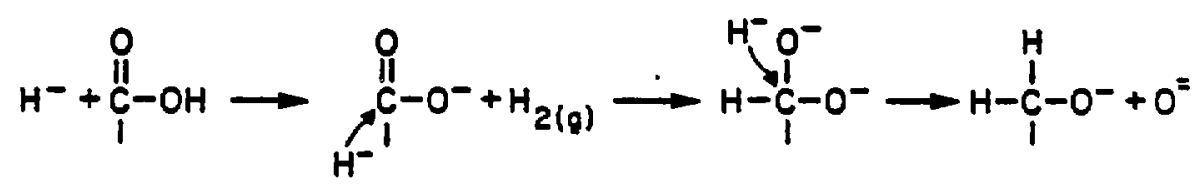<smiles>[CH-]C(C)(C)[CH-]C(C)(C)C</smiles>

$\mathrm{H}^{-}+-{ }_{\mathrm{C}}^{\mathrm{C}}-\mathrm{OH} \rightarrow-\mathrm{C}^{\mathrm{C}}-\mathrm{O}^{-}+\mathrm{H}_{2(0)}$

Figure 4.3 Reactions of Selected Functional Groups with Lithium Alumiaum Hydride. 
diglyme to maintain a reaction temperature near ambient. Sealing the flask increased the internal pressure, so the serum stopper was pierced momentarily with a syringe needle to bring the pressure back to ambient. Twenty milliliters of the $\mathrm{LiAlH}_{4}$-diglyme solution were injected through the serum stopper and vigorous stirring was initiated. Manometer readings were taken periodically for about two hours to determine the amount of hydrogen evolved.

Blank runs were required to interpret the data properly. Immediately before an adsorbent was tested, a trial run was carried out with no adsorbent. Adding reagent to the flask increased the internal pressure and, more importantly, hydrogen gas was evolved from the

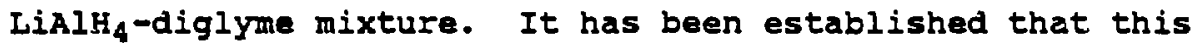
type of solution reacts slowly with atmospheric oxygen (Hochstein, 1949) and that water in the solvent would react. It was important to run the blank and the trial with adsorbent in quick succession and as soon as possible after preparation of the $\mathrm{LiAlH}_{4}$-diglyme solution. Lithium aluminum hydride converts to an insoluble and nonreactive form in ether solution (Brown, 1951). The difference in the amount of hydrogen evolved from a run with an adsorbent, and that evolved over the same time period for a blank run, was used to calculate the amount of active hydrogen on the adsorbent surface. 
There are other complications in the determination of active hydrogen. One concern is that $\mathrm{LiAlH}_{4}$ reacts with water to form hydroxl ion and hydrogen gas. Carbons were carefuily dried before the measurements were made, and mixtures of $\mathrm{IiAlH}_{4}$ and diglyme were prepared under a dry nitrogen atmosphere. It has also been observed that the amount of active hydrogen determined experimentally does not always correspond to the value suggested by the chemical structure (Krynitsky et al., 1948). In particular, aromatic diols liberate an average of 1.5 rather than 2 equivalents of $\mathrm{H}_{2}(g)$. This fact suggests that keto-enol tautomerization and other resonance effects may be important for the polynuclear aromatic surface of an activated carbon. Despite these complications, active hydrogen should be a useful measure of the total surface acidity.

Table 4.2 lists active hydrogen values for four of the vendor-supplied carbons on per gram and per unit surface area bases. Munson (1985) determined the values for super Sorb and for Row 0.85 , but did not report a value for Witcarb 950. The values for Columbia and $F-100$ ware determined in this work. The active hydrogen contents reported on a per gram basis are in the same order as the hydrogen waight percents determined by elemental analysis (see Table 4.1). Except for the case of Fr-100, the active hydrogen values are less than the amount of hydrogen 
Table 4.2 Active Hydrogen Contents of Vendor-Supplied Activated Carbons

\begin{tabular}{|c|c|c|}
\hline \multirow[b]{2}{*}{ Activated Carbon } & \multicolumn{2}{|c|}{ Active Hydrogen } \\
\hline & (mmoles $/ \mathrm{g})$ & ( $\left.\mu \mathrm{mole} / \mathrm{m}^{2}\right)$ \\
\hline Super Sorb & 1.12 & 0.47 \\
\hline Row $0.8 \mathrm{~s}$ & 3.10 & 4.77 \\
\hline Columbia & 0.88 & 0.98 \\
\hline$F=100$ & 0.78 & 0.97 \\
\hline
\end{tabular}


indicated by elemental analysis. For F-100, the actual weight percent of hydrogen is probably higher than the value reported in Table 4.1. This contention is supported by the fact that elemental analysis gave 0.82 wt. $\%$ hydrogen in a sample of F-100 that was rinsed with water but not subjected to Soxhlet extraction (Munson, 1985).

\subsubsection{Tourier Transform Infrared spectroscopy}

Two of the vendor-supplied activated carbons used in this work had previously been characterized by Fourier Transform Infrared spectroscopy (FTIR). In addition, a carbon treated with nitric acid was characterized, and the results are significant to this study. The analyses were conducted by Curtis Munson and are described in his Ph.D. dissertation (1985). Relevant conclusions are presented in this section.

An FTIR spectrum for Witco Columbia showed two peaks -one at wavenumber ca. $1530 \mathrm{~cm}^{-1}$ and one at wavenumber ca. $1100 \mathrm{~cm}^{-1}$. The former peak could indicate enol carbonyl, guinone, and/or graphitic aromatic functionality. The latter peak could indicate phenolic or amine functionality. Since Witco Columbia has a low nitrogen content, phenolic groups are indicated. This view is supported by the sizable amounts of oxygen and active hydrogen and the fact 
that there was no (FTIR) evidence for carboxylic acid functional groups (i.e. there were no peaks at ca. 1720 and $1220 \mathrm{~cm}-1)$.

oxidation of witco Columbia with nitric acid (for 3 hours at $50^{\circ} \mathrm{C}$ ) dramatically altered the surface chemistry. The spectrum showed sharp peaks at 1720,1580 , and 1220 $\mathrm{cm}^{-1}$. Munson decided that carboxylic acid and/or dicarboxylic acid groups were predominant and that some oxygen was present in the forth of carbonyls or ethers.

In contrast, carbonyl and phenolic groups predominate on the surface of Norit Row 0.8s. The FTIR spectrum showed a strong band centered at $1570 \mathrm{~cm}^{-1}$ and weaker bands at 1640, 1240, and $1120 \mathrm{~cm}^{-1}$. The strong bend indicates carbonyl groups, while there was no evidence for carboxylie acid groups. The amount of active hydrogen and the band at $1240 \mathrm{~cm}^{-1}$ suggest the presence of phenolic groups. In addition there was evidence for amine functionality.

\subsubsection{Influences of Functional groups on Irreversible Adsorption}

A popular hypothesis used to explain irreversible adsorption of phenolics on activated carbon is cheinical reaction of sorbate molecules with surface functional groups (Yonge, 1985; Modell, et al., 1980; Puri, 1980; and chang, 1980 \& 1981). Several experiments designed to test this hypothesis are presented in this section. In these 
experiments, the adsorption capacities and regenerabilities of adsorbents with different surface chemistries are compared.

\subsubsection{Experimental Procedure}

Unless otherwise specified, the contact conditions were as follows: A known weight of $20 \times 40$ mesh size adsorbent was placed in a $20 \mathrm{ml}$ scintillation vial along with $15 \mathrm{ml}$ of an aqueous solution containing a phenolic solute. The vial was sealed with a teflon-lined cap and was maintained at a constant temperature without agitation. After 5 days of contact the teflon-lined cap was replaced by a cap that was perforated and that contained a 0.5 micrometer Filinert membrane filter (Nuclepore Corp.). The filter had been prewet with acetone, thoroughly rinsed with water, and then blotted 'dry' with a kimwipe tissue. A plastic centrifuge tube was inverted and placed over the top of the vial, and the combination was sealed with parafilm. The connected vial and tube were placed in a Damon/IEC model CL centrifuge, tube side down, and spun at $2500 \mathrm{rpm}$ for 10 minutes. The aqueous phase was decanted from the centrifuge tube, and the tube and the vial were reconnected. The combination was returned to the centrifuge, tuibe side up, and spun lightly for about a minute. This step separated the carbon from the moist filter. The filter was removed from the cap and placed in the vial. Acetone was introduced to the vial, and batch 
regenerations were performed until the cumulative volume (in $\mathrm{ml}$ ) of acetone used was atleast 500 times the dry adsorbent weight (in grams), or until no phenolic compound could be detected in the regene-ant after at least two days of contact. The scintillation vial was agitated at ambient temperature during the regeneration contacts. After each. such contact the regenerant mixture was separated from the carbon by centrifugation in the same manner as was the aqueous phase. The same filter was used for each centrifugation of an adsorbent sample.

Tests were done to ensure that this procedure yielded adequate mass balances. Aqueous solutions of phenol did not change concentration when contacted with a plastic centrifuge tube, and phenol could not be detected in the regenerant when a trial was performed without an adsorbent (but with a filter). In addition, mass balances closed well for trials with $X A D-4$ and phenol or resorcinol (see Section 4.1 .1 ).

\subsubsection{Vendor-supplied carbons}

Several investigators have stated or implied that irreversible adsorption is due to a 1:1 stoichiometric reaction between sorbate molecules and oxygen-containing surface functional groups (Yonge, 1985; Modell, et al., 1980: Puri, 1980: and Chang, 1980 \& 1981). An experiment 
designed to compare the tendencies of the vendor-supplied carbons to adsorb phenol irreversibly shows that such an explanation is inadequate.

Equal surface areas $\left(30 \mathrm{~m}^{2}\right)$ of the carbons were contacted with equal volume aliquots of an aqueous solution $6.27 \times 10^{-3} \mathrm{M}$ in phenol. Two trials were run for each carbon, and the adsorption temperature was $80^{\circ} \mathrm{C}$.

The second and third columns in Table 4.3 show the average amounts of total and irreversible adsorption per unit surface area of each carbon and the variations from the averages. In each case, between one-half and two-thirds of the adsorption could not be reversed. Irreversibly sorbed phenol molecules cover large fractions of the carbon surfaces 4 . If phenol is adsorbed irreversibly on particular sites, then, those sites must be very abundant. Further, since high loadings of reversibly sorbed phenol can be achieved on the same carbons, physisorption and chemisorption can occur on the same type of site.

The fourth and fifth columns show maximum surface concentrations of oxygen and heteroatoms (oxygen, sulfur,

1 There is disagreement in the literature about the area occupied by a phenol molecule at various surface concentrations. If a flat orientation is assumed, a molecular area of $0.45 \mathrm{~nm}^{2}$ is reasonable (Mattson and Mark, 1971 and Chang et $a l ., 1981)$, and 3.7 micromoles of phenol would cover $1 \mathrm{~m}^{2}$ of surface. On this basis, irreversibly adsorbed phenol covers 28 to $43 \%$ of the surface area of the vendor-supplied carbons. 
Table 4.3 Comparative Regenerability of Vendor-Supplied Activated Carbons

\begin{tabular}{lcccc}
$\begin{array}{c}\text { Activated } \\
\text { Carbon }\end{array}$ & $\begin{array}{c}\text { Total } \\
\text { Adsorption } \\
\left(\mu \text { moles } / \mathrm{m}^{2}\right)\end{array}$ & $\begin{array}{c}\text { Irreversible } \\
\text { Adsorption } \\
\left(\mu \text { moles } / \mathrm{m}^{2}\right)\end{array}$ & $\begin{array}{c}\text { Oxygen } \\
\left(\mu \mathrm{moles} / \mathrm{m}^{2}\right)\end{array}$ & $\begin{array}{c}\text { Hereroatoms } \\
\left(\mu \mathrm{moles} / \mathrm{m}^{2}\right)\end{array}$ \\
\hline Super Sorb & $1.73 \pm .02$ & $1.02 \pm .01$ & 0.20 & 0.25 \\
Witcarb 950 & $2.06 \pm .05$ & $1.05 \pm .05$ & 0.54 & 1.39 \\
F-100 & $2.33 \pm .03$ & $1.55 \pm .08$ & 2.66 & 3.33 \\
Row 0.8s & $2.48 \pm .02$ & $1.56 \pm .07$ & 9.88 & 10.63 \\
Columbia & $2.47 \pm .02$ & $1.58 \pm .05$ & 1.67 & 2.82 \\
\hline
\end{tabular}

"Maximum surface concentrations based on elemental analysis. 
and nitrogen) for each of the carbons. These concentrations were calculated from the elemental analyses . with the amounts of oxygen for Columbia and F-100 determined from nonclosure of the mass balances. These are maximum surface concentrations, because using mass balance nonclosures probably over estimates the oxygen values and because some of the heteroatoms will not be at the carbon surfaces. The latter point is particularly important for Row 0.83 , which is derived from peat and is expected to have a large amount of internal oxygen.

Comparison of the third and fourth columns shows that for Super Sorb and Witcarb 950 there is not enough oxygen to account for the irreversible adsorption by one or more 1:1 reactions. This would also be true for columbia if the oxygen analysis reported by Munson was used. Groups containing sulfur or nitrogen might also be reactive, but, as comparison of the third and fifth columns shows, $1: 1$ stoichiometric reactions would still not account for the amount of irreversible adsorption on super sorb.

The relative tendencies of the vendor-supplied carbons to adsorb phenol have additional implications. There does not seem to be a direct relationship between total or irreversible adsorption and surface acidity as measured by active hydrogen. Further, despite wide differences in the elemental analyses, the last three carbons listed in Table 4.3 had virtually identical adsorption tendencies. The. 
amounts of oxygen or total heteroatoms on the carbons did not correlate well with total or irreversible adsorption. Neither did the ash contents of the carbons seem to matter. In sum, this "broad-brush" experiment does not indicate a mechanism for irreversible adsorption, but it does make some of the possibilities less likely.

\subsubsection{3 oxidised carbons}

Surface functional groups form on an activated carbon surface that is exposed to an oxidizing solution of sufficient strength. Witco Columbia was treated with various oxidants in aqueous solutions in order to develop a variety of surface chemistries. Hydrogen peroxide, nitric acid, and potassium permanganate were the oxidants employed. Potassium permanganate added substantial amounts of ash to the carbon. Consequently the treatment results for $\mathrm{KMnO}_{4}$ are discussed in section 4.1.5.

Hydrogen peroxide is a relatively weak oxidizing agent. According to Schirmann and Delavarenne (1979), $\mathrm{H}_{2} \mathrm{O}_{2}$ is too weak to oxidize aromatic compounds (dissolved in solution) unless the compounds are 'activated' or unless activators or catalysts are employed. Under neutral or acidic conditions oxidation proceeds through electrophilic addition of $\mathrm{OH}^{+}$, and electron donating substituents activate an aromatic nucleus. That is, electron donating groups help to stabilize the positive charge on a reaction 
intermediate. With concentrated $\mathrm{H}_{2} \mathrm{O}_{2}$ and a strong acid, oxidation can proceed past the addition of hydroxyl groups to the formation of quinones and even to ring opening. Under basic conditions, hydroxyl groups may be introduced through nucleophilic substitutions. As examples, aromatic ketones and aldehydes may be converted to the corresponding phenols.

oxidation of activated carbon with hydrogen peroxide probably involves a number of reactions. The surface is activated by virtue of the polynuclear aromatic structure, and existing functional groups may be oxidized. In addition, activated carbon catalyzes the decomposition of $\mathrm{H}_{2} \mathrm{O}_{2}$, and the adsorption characteristies of the products are not well understood (Puri, 1970). It has been shown, however, that reaction of carbon blacks with $3 \mathrm{~N} \mathrm{H}_{2} \mathrm{O}_{2}$ results in surface functional groups that evolve carbon dioxide upon heating (Puri, 1966). Evolution of $\mathrm{CO}_{2}$ is thought to indicate carboxylic or lactone groups rather than phenolic groups (Riven, 1963).

Four aqueous solutions containing $\mathrm{H}_{2} \mathrm{O}_{2}$ were used to oxidize samples of witco columbia. The solutions contained 3 wt. $\mathrm{H}_{2} \mathrm{O}_{2}, 3$ wt. $\mathrm{H}_{2} \mathrm{O}_{2}$ and $0.15 \mathrm{~N} \mathrm{HF}, 3$ wt. $\mathrm{H}_{2} \mathrm{O}_{2}$ and 0.9 $\mathrm{N} \mathrm{NaOH}$, and $30 \mathrm{wt.} \mathrm{H}_{2} \mathrm{O}_{2}$. Nagalene or other polymeric labware was used to prepare and to hold the solution containing hydrogen fluoride. About 4 or 5 grams of carbon were added to $200 \mathrm{ml}$ of each of the solutions, and the 
mixtures were agitated mildly for 1 day. The oxidized carbons were then rinsed with water and subjected to the precreatment procedure described in section 2.1 .

Elemental analyses and initial and final weights of the carbons are summarized in Table 4.4. Weight changes were small for carbons treated with $3 * \mathrm{H}_{2} \mathrm{O}_{2}$ in basic, neutral, or acidic solutions, although hydrogen fluoride created a large amount of eines. As expected, changes in the oxygen contents of the treated carbons (as indicated by nonclosure of the mass balances) are much more dramatic than changes in other heteroatoms. Since there was not a source of nitrogen during the oxidation, there is probably a small amount of nitrogen in untreated columbia, and variations in nitrogen contents of the samples are probably negligible. The reduced sulfur content (and the reduced surface area, see Table 4.5) of the carbon oxidized with $30+\mathrm{H}_{2} \mathrm{O}_{2}$ probably reflects material losses due to gasification.

Surface areas of the carbons are listed in Table 4.5, as are ratios of added oxygen to added elemental hydrogen. The numerators and denominators of the ratios are in units of moles $/ \mathrm{m}^{2}$ so that the ratios indicate relative changes in the surface densities of the elements. Tr 2 ratios are very close to unity for the carbons treated with $3+\mathrm{H}_{2} \mathrm{O}_{2}$ and with 3* $\mathrm{H}_{2} \mathrm{O}_{2}$ and NaOH. This fact suggests the addition of phenolic groups without oxidation of functional groups originally on the surface of witco columbia. The ratio is 
Tabic 4.4 Weight Changes and Elemental Analyses for Oxidized Columbia

Elemental Analyses (Weight Perecnt)

\begin{tabular}{|c|c|c|c|c|c|c|c|c|c|}
\hline \multirow[b]{2}{*}{$\begin{array}{l}\text { Oxidizing } \\
\text { Solution }\end{array}$} & \multirow[b]{2}{*}{$\begin{array}{l}\text { Initial } \\
\text { Weight } \\
\text { (g) }\end{array}$} & \multirow[b]{2}{*}{$\begin{array}{l}\text { Final } \\
\text { Weight } \\
\text { (g) }\end{array}$} & \\
\hline & & & $\mathrm{C}$ & $\mathrm{H}$ & $N$ & $S$ & Ash & Total & $0^{\circ}$ \\
\hline None & $N A^{*-}$ & NA" & 93.70 & 0.18 & 0.0 & 3.31 & 0.4 & 97.59 & 2.41 \\
\hline $\begin{array}{l}3 \% \mathrm{H}_{2} \mathrm{O}_{2} \\
0.9 \mathrm{~N} \mathrm{~N}\end{array}$ & 5.100 & 5.099 & 93.07 & 0.23 & 0.06 & 3.12 & 0.3 & 96.78 & 3.29 \\
\hline $\begin{array}{l}3 \% \mathrm{H}_{2} \mathrm{O}_{2} \& \\
0.15 \mathrm{~N}^{2} \mathrm{H}^{2}\end{array}$ & 5.057 & 5.101 & 92.16 & 0.37 & 0.08 & 3.37 & 0.6 & 96.58 & 3.42 \\
\hline $3 \% \mathrm{H}_{2} \mathrm{O}_{2}$ & 3.921 & $3.86 !$ & 88.67 & 0.42 & 0.17 & 3.02 & 0.9 & 93.18 & $0.8=$ \\
\hline $30 \% \mathrm{H}_{2} \mathrm{O}_{3}$ & 4.837 & 3.978 & 83.39 & 0.33 & 0.09 & 1.93 & 0.4 & 86.14 & 13.86 \\
\hline $\begin{array}{l}70 \% \text { HNO } \\
2 t 50 \circ \mathrm{C}\end{array}$ & ND". & ND" & 85.65 & 0.18 & 0.65 & 2.41 & 0.1 & 88.99 & 11.01 \\
\hline
\end{tabular}

Calculated by difference

- Not Applicable or Not Determined

Table 4.5 Surface Areas and Oxygen*/Hydrogen Ratios for Oxidized Columbia

\begin{tabular}{|c|c|c|}
\hline $\begin{array}{l}\text { Treatment } \\
\text { Solution }\end{array}$ & $\begin{array}{c}\mathrm{N}_{2} \text { BET } \\
\text { Surface Areas } \\
\left(\mathrm{m}^{2} / \mathrm{gdc}\right)\end{array}$ & $\stackrel{2}{\mathrm{JH}}^{* \bullet}$ \\
\hline None & 900 & $\ldots$ \\
\hline $\begin{array}{l}3 \% \mathrm{H}_{2} \mathrm{O}_{2} \& \\
0.9 \mathrm{~N} \mathrm{NaOH}\end{array}$ & 900 & 1.01 \\
\hline $\begin{array}{l}3 \% \mathrm{H}_{2} \mathrm{O}_{2} \& \\
0.15 \mathrm{~N}^{2} \mathrm{~F}\end{array}$ & 930 & 0.32 \\
\hline $3 \% \mathrm{H}_{2} \mathrm{O}_{2}$ & 930 & 1.16 \\
\hline $30 \% \mathrm{H}_{2} \mathrm{O}_{2}$ & 830 & 4.44 \\
\hline $\begin{array}{l}70 \% \mathrm{HNO}_{3} \\
\text { at } 50 \circ \mathrm{C}\end{array}$ & 835 & 42.2 \\
\hline
\end{tabular}

"All oxygen values were determined by difference from elemental analyses.

- Ratio of added oxygen (mole/ $\mathrm{m}^{2}$ ) to added elemental hydrogen (mole $/ \mathrm{m}^{2}$ ). 
mucis lower than 1 for the carbon treated with $3 t \mathrm{H}_{2} \mathrm{O}_{2}$ and HF, but the reasons for this are not apparent. The ratio is greater than 4 to 1 for the carbon treated with 308 $\mathrm{H}_{2} \mathrm{O}_{2}$, indicating that surface functional groups were oxidized to forms containing little hydrogen.

Table 4.6 compares adsorption characteristics of these treated carbons (along with another to be discussed) and witco Columbia. Equal surface areas $\left(24 \mathrm{~m}^{2}\right)$ of the carbons were contacted with $15 \mathrm{ml}$ of an aqueous solution $6.38 \mathrm{x}$ $10^{-3} \mathrm{M}$ in phenol. The contact time was 5 days, and the temperature was $80^{\circ} \mathrm{C}$. Two samples of each carbon were used, and two trials (4 samples total) were run for Columbia oxidized with $3 t \mathrm{H}_{2} \mathrm{O}_{2}$. The second trial had better temperature control and is probably more reliable than the first. The second and third columns of Table 4.6 show that oxidation decreased the specific capacity of Columbia. The second column lists total adsorption for each carbon, and the third column lists differences between the total adsorption amounts for the treated and untreated carbons. The next two colusns show that there are even greater decreases in the amounts of irreversible adsorption. The fifth column lists the differences between the values for the treated and the untreated carbons. The last column shows that the oxidized carbons are percent-wise more regenerable than untreated columbia. 
Table 4.6 Comparative Regenerability of Columbia and Oxidized Columbia

\begin{tabular}{|c|c|c|c|c|c|}
\hline \multirow[b]{2}{*}{$\begin{array}{l}\text { Oxidizing } \\
\text { Solution }\end{array}$} & \multicolumn{4}{|c|}{ Adsorption ( $\mu$ mole $/ \mathrm{m}^{2}$ ) } & \multirow[b]{2}{*}{$\begin{array}{c}\text { Percent } \\
\text { Regeneration }\end{array}$} \\
\hline & Total & $\Delta$ Total & Irrev." & د Irrev." & \\
\hline None & $2.51 \pm .03$ & $\cdots$ & $1.62 \pm .04$ & $-\cdots$ & $35.7 \pm 1.2$ \\
\hline $\begin{array}{l}3 \% \mathrm{H}_{2} \mathrm{O}_{2} \& \\
0.9 \mathrm{~N} \mathrm{NaOH}\end{array}$ & $2.39 \pm .06$ & -0.12 & $1.44 \pm .01$ & -0.18 & $39.7 \pm 1.0$ \\
\hline $\begin{array}{l}3 \% \mathrm{H}_{2} \mathrm{O}_{2} \& \\
0.15 \mathrm{~N} \mathrm{HF}\end{array}$ & $2.23 \pm .03$ & -0.28 & $1.24 \pm .00$ & -0.38 & $44.3 \pm 0.6$ \\
\hline $\begin{array}{c}3 \% \mathrm{H}_{2} \mathrm{O}_{2} \\
\text { Trial } 1 \\
\text { Trial } 2\end{array}$ & $\begin{array}{l}2.21 \pm .02 \\
2.03 \pm .02\end{array}$ & $\begin{array}{l}-0.30 \\
-0.48\end{array}$ & $\begin{array}{l}1.27 \pm .04 \\
1.04 \pm .02\end{array}$ & $\begin{array}{l}-0.35 \\
-0.58\end{array}$ & $\begin{array}{l}42.8 \pm 0.9 \\
48.5 \pm 1.1\end{array}$ \\
\hline $30 \% \mathrm{H}_{2} \mathrm{O}_{2}$ & $1.40 \pm .02$ & -1.11 & $0.27 \pm .01$ & -1.35 & $80.9 \pm 0.6$ \\
\hline $\begin{array}{l}70 \% \mathrm{HNO}_{3} \\
\text { at } 50 \circ \mathrm{C}\end{array}$ & $1.77 \pm .05$ & -0.74 & $0.36 \pm .05$ & -1.26 & $79.7 \pm 2.7$ \\
\hline
\end{tabular}

Irreversible 
The adsorption results for the carbons oxidized with hydrogen peroxide are revealing. The greater the amount of oxygen added to the surface, the lower the specific capacity and the more regenerable the carbon. Further, the primary influence of oxidation is on the irreversible portion of the adsorption; total and irreversible adsorption decrease by similar amounts 5 . The functional groups (or surface complexes; added to the carbons do not promote chemical reactions involving phenol. In fact, these results indicate that phenolic groups and carbonyl groups (quinones) are not reactive, although the chemical characterization of these groups was not definitive.

Witco Columbia was also oxidized with $70 \%$ nitric acid. Several grams of carbon were immersed in acid in a round bottom flask and the mixture was heated to $\mathrm{ca} .50^{\circ} \mathrm{C}$ for three hours. The oxidation appeared much more vigorous than it appeared for the mixtures containing hydrogen peroxide. The oxidized carbon was rinsed several times with water and then subjected to the pretreatment procedure described in section 2.1.

Carboxylic acid and carbonyl groups should form on the surface of columbia during treatment with nitric acid.

5 When comparing uptakes on different adsorbents, note that the driving force for physisorption is higher when the total uptake is lower. This is because, the higher the uptake, the lower the concentration of solute in bulk solution. This fact favors smaller decreases in total adsorption than in irreversible adsorption. 
This contention is supported by the work of Munson (1985) as discussed in section 4.1.3.3. Munson oxidized columbia with $\mathrm{HNO}_{3}$ for 3 hours at $50^{\circ} \mathrm{C}$, but did not follow the oxidation procedure with soxhlet extractions. An FTIR spectrum showed peaks corresponding to carboxylic acid and/or dicarboxylic acid groups, and Munson argued that some of the surface oxygen was also in the form of carbonyls or ethers. The formation of carboxylic and dicarboxylic acid groups on carbon is consistent with solution-phase reactions in which nitric acid cleaves aromatic rings (cotton and wilkinson, 1980). The formation of carbonyl (actually quinone) groups is supported by the work of Reiss and Donnet (2964) with carbon blacks.

Nitric acid may influence the surface chemistry of carbons in other ways. In particular, Puri and coworkers (1964, 1966, 1970 and others) have argued that a chemisorbed " $\mathrm{CO}_{2}$-complex" forms on carbon blacks and on charcoal when they are oxidized with concentrated $\mathrm{HNO}_{3}$. Such a complex would contribute to surface acidity as measured by titration with strong base, but would not contribute to the hydrogen content of a carbon. In contrast, Garten and Weiss (1957a) have argued that nitric acid oxidation promotes lactones on carbon surfaces.

Elemental analyses and the nitrogen BET surface area for Columbia treated with $\mathrm{HNO}_{3}$ are summarized in Tables 4.4 and 4.5. Initial and final weights were not determined 
because some of the material was lost as fines, but oxidation was vigorous and some of the carbon was gasified. The elemental analyses show that a large amount of oxygen and a small amount of nitrogen were added to the surface. The strong acid treatment reduced the sulfur and ash contents of the carbon, and the surface area decreased by about 7f. There was a small increase (ca. 87) in the surface density of elemental hydrogen.

The elemental analyses provide clues about the surface functionality, but they are not sufficient to determine the nature of the added oxygen. The ratio of added oxygen to added hydrogen indicates that most of the oxygen is present in forms that do not contain hydrogen. Carbonyl groups, lactones, and $\mathrm{CO}_{2}$-complexes are all possible forms of the added oxygen.

Batch adsorption/regeneration runs were carried out for two samples of $\mathrm{HNO}_{3}$-oxidized Columbia. The contact conditions were the same as those used for the carbons treated with $\mathrm{H}_{2} \mathrm{O}_{2}$. The results are summarized in Table 4.6 and are consistent with several trends already noted. In particular, total and irreversible adsorption both decreased, and the amounts were between those for columbia treated with 3 : $\mathrm{H}_{2} \mathrm{O}_{2}$ and for Columbia treated with $30 \%$ $\mathrm{H}_{2} \mathrm{O}_{2}$. These results are consistent with the relative oxygen contents of the carbons. Oxidation with nitric acid added functional groups to this surface, but the groups did 
not promote irreversible adsorption. Rather, Columbia treated with $\mathrm{HNO}_{3}$ is far more regenerable than the parent carbon.

\subsubsection{Carbons Treated by other Methods}

Three additional treatment methods were used to alter the surface functionality of columbia carbon. One sample was reduced with $\mathrm{L}_{1 A 2 H_{4}}$, another was oxidized with nitric acid and then contacted with ammonia at $600{ }^{\circ} \mathrm{C}$, and a third was contacted with sulfur dioxide at $510^{\circ} \mathrm{C}$. The surface chemistries and the tendencies of the treated carbons to adsorb irreversibly varied greatly.

Lithium aluminum hydride reduces surface functional groups on carbons as discussed in Section 4.1.3.2. A 5 gram sample of $20 \times 40$ mesh (pretreated) Columbia was immersed in $100 \mathrm{ml}$ of a freshly prepared solution of $\mathrm{LiAlH}_{4}$ (5 g) in diglyme (100 $\mathrm{ml})$ for ca. 4 hours. The reacted carbon was rinsed with diglyme and then with water. The carbon was subjected to soxhlet extractions with methansl and with water and then dried.

Elemental analyses of the reduced and the parent carbons are listed in Table 4.7. The reduced carbon has higher weight fractions of hydrogen and of ash than did the parent carbon. Presumably, the additional ash is due to lithium and/or aluminum (perhaps in the form of an insoluble ether complex). Aluminum in the form of hydrides 
Table 4.7 Elemental Analyses for Columbia Treated in Various Ways

\begin{tabular}{|c|c|c|c|c|c|c|c|}
\hline \multirow[b]{2}{*}{ Treatment } & \multicolumn{7}{|c|}{ Weight Percent } \\
\hline & $\mathrm{C}$ & $\mathrm{H}$ & $\bar{N}$ & $\mathrm{~S}$ & Ash & Total & $0^{\circ}$ \\
\hline None & 93.70 & 0.18 & 0.0 & 3.31 & 0.4 & 97.59 & 2.41 \\
\hline $\mathrm{LiAlH}_{4}$ & 88.93 & 0.43 & 0.09 & 3.09 & 2.8 & 95.34 & 4.66 \\
\hline $\begin{array}{l}\mathrm{HNO}_{3} \& \\
\mathrm{NH}_{3} \text { at } \\
600{ }^{\circ} \mathrm{C}\end{array}$ & 67.92 & 0.57 & 0.58 & 1.05 & 0.10 & 70.22 & $29.7 \mathrm{~s}$ \\
\hline $\begin{array}{l}\mathrm{SO}_{2} \text { at } \\
510^{\circ} \mathrm{C}\end{array}$ & 89.20 & 0.0 & 0.0 & 7.23 & 0.8 & 97.23 & 2.77 \\
\hline
\end{tabular}

"Calculated by difference 
would increase the weight percent of hydrogen. The FTIR spectrum of columbia indicated the presence of carbonyl groups (see section 4.1 .3 .3 ). The hydrogen content of columbia would be increased by reduction of carbonyl groups to phenolic groups.

The elemental analyses also indicate increases in nitrogen and oxygen. As stated in the previous section (4.1.4.3) small variations in the nitrogen weight percent are judged to be insignificant. The reasons for the increased oxygen content are not apparent.

Reduction of Columbia with $\mathrm{LiAlH}_{4}$ did not change the specific surface area. Nitrogen BET surface areas for the two carbons are included in Table 4.8 .

Table 4.9 shows the results of batch adsorption/regeneration tests for the reduced and the parent carbons. The experimental conditions for all tests in Table 4.9 were those described in connection with Table 4.6. The carbon loadings (total adsorption) for columbia and the reduced carbon are virtually identical, but the reduced carbon is more regenerable. Qualitatively, the increase in regenerability is in line with the increased oxygen content of the surface. That is, the reduced carbon follows the trend observed for oxidized carbons. The quantity, rather than the identity, of the functional groups seems to have the most influence on the reversibility of phenol adsorption. 
Table 4.8 Surface Areas for Columbia Treated in Various Ways

\begin{tabular}{|c|c|}
\hline Trestment & $\begin{array}{c}N_{2} \text { BET Surface Areas } \\
\left(\mathrm{m}^{2} / \mathrm{gdc}\right)\end{array}$ \\
\hline None & 900 \\
\hline $\mathrm{LiAlH}_{4}$ & 900 \\
\hline $\mathrm{HNO}_{6} \& \mathrm{NO}_{0} \mathrm{NH}_{3} \mathrm{Ct}$ & 280 \\
\hline $\mathrm{SO}_{2}$ at $510^{\circ} \mathrm{C}$ & 900 \\
\hline
\end{tabular}

Table 4.9 Comparative Regenerability of Columbia Treated in Various Ways

\begin{tabular}{|c|c|c|c|c|c|}
\hline \multirow[b]{2}{*}{ Treatment } & \multicolumn{4}{|c|}{ Adsorption ( $\mu \mathrm{mole} / \mathrm{m}^{2}$ ) } & \multirow[b]{2}{*}{$\begin{array}{c}\text { Percent } \\
\text { Regelicration }\end{array}$} \\
\hline & Total & $\Delta$ Total & Irrev.: & دIrrev." & \\
\hline None & $2.51 \pm .03$ & -... & $1.62 \pm .04$ & ... & $35.7 \pm 1.2$ \\
\hline $\mathrm{LiAlH}_{4}$ & $2.52 \pm .04$ & 0.01 & $1.47 \pm .01$ & -0.15 & $41.4 \pm 0.3$ \\
\hline$\underset{\text { at } 600 \cdot \mathrm{C}}{\mathrm{HNO}_{3} \& \mathrm{NH}_{3}}$ & $1.16 \pm .06$ & -1.35 & $0.40 \pm .05$ & -1.22 & $65.3 \pm 2.4$ \\
\hline $\mathrm{SO}_{2}$ at $510^{\circ} \mathrm{C}$ & $2.38 \pm .06$ & -0.13 & $1.26 \pm .03$ & -0.36 & $47.1 \pm 0.1$ \\
\hline
\end{tabular}

"Irreversible 
Another sample of Columbia was oxidized with

concentrated nitric acid and then reacted with ammonia at $600^{\circ} \mathrm{C}$. The treatment was carried out by Dr. Yue Kuo, a former member of our Berkeley research group. The contact with nitric acid was at $50^{\circ} \mathrm{C}$ for several hours, and the carbon was washed thoroughly before it was contacted with ammonia (Garcia, 2987). The oxidized carbon was reacted with ammonid for approximately 10 hours at $6000^{\circ} \mathrm{C}$. The carbon was exposed to the atmosphere during Dr. Kuo's work and then was stored in a sealed flask for approximately 4 years. The carbon was ground and seived to $20 \times 40$ mesh and then subjected to the pretreatment procedure described in Section 2.1 .

Elemental analyses for this carbon are summarized in Table 4.7. A relatively small amount of nitrogen was introduced to columbia, probably in the form of basic functional groups (i.e. immines, amides, and/or nitriles; Kuo, 2983). The oxygen content is very high, and oxygen containing functional groups should dominate the surface chemistry of the treated carbon. Significant amounts of hydrogen were added to the parent carbon, and sulfur and ash were lost.

The surface area of the $\mathrm{HNO}_{3} / \mathrm{NH}_{3}$ treated carbon is very low, as shown in Table 4.8. Extensive oxidation probably widened (and eliminated) many of the micropores. 
Batch adsorption/regeneration trials were conducted with two samples of this carbon in the same unanner as for the other treated carbons. The results are included in Table 4.9 and show that total and irreversible adsorition of phenol were much lower than for carbons with lower oxygen contents. The very hydrophilic surface had relatively little affinity for phenol and in this case, total adsorption decreased by slightly more than did irreversible adsorption. The treated carbon was more regenerable than the parent carbon, but not as regenerable as the carbon treated with nitric acid alone. This fact indicates that the nature as well as the abundance of functional groups influences irreversible adsorption.

Columbia was also treated with sulfur dioxide at $510^{\circ} \mathrm{C}$. The treatment procedure was carried out by Dr. Tony Garcia and is detailed in his Ph.D. dissertation (Garcia, 1988). After reaction with $\mathrm{SO}_{2}$ for 9 hours, the modified carbon was cooled in a flow of helium. The carbon was then exposed to the atmosphere for approximately nine months.

Garcia found that Columbia treated in a similar way developed sulfur-oxide functional groups of unknown identity, but retained much of its original hydrophobic surface area. He also contended that at least some of the sulfur-oxygen groups were strong Brönstead acids. 
Results of elemental analyses for the $\mathrm{SO}_{2}$ treated carbon appear in Table 4.7. There is a substantial increase in the sulfur content of the carbon, but not in the oxygen content. It is likely, however, that much of the oxygen present on Columbia was removed at $510^{\circ} \mathrm{C}$, and that oxygen on the treated carbon is combined with sulfur in various groups (Garcia, 1987). The treated carbon has a very low hydrogen content, which suggests the absence of Brönstead acidity. This carbon (and those discussed by Garcia (1988)) may have substantial Lewis acidity, however.

Table 4.9 show that the $\mathrm{SO}_{2}$ treated carbon has less tendency to sorb phenol irreversibly than did the parent carbon. Considering the dramatic changes in surface chemistry, however, the changes in adsorption amounts are small.

The three treatment procedures discussed in this section developed widely different surface chemistries on the same parent carbon. The treated carbons had very different tendencies to sorb phenol irreversibly, but the differences were probably due more to the abundance than to the nature of the surface functional groups.

\subsubsection{Functional Groups on Polymeric Resias}

Many polymeric adsorbents containing particular functional groups are marketed. These resins are generally well characterized, making their interactions with sorbates 
easier to interpret. Monitoring the reversibility of adsorption of phenolic compounds on such resins may help to define the role of individual functional groups in promoting irreversible xdsorption on activated carbon.

Several investigators have used on' or more functionalized polymers to remove phenolic compounds from water (e.g. Wojaczynska et. al, 1984; Farrier et. al, 1979; Kim et. al, 1976; and Chasanov et. al, 1956), but relatively few have tested the reversibility of adsorption. Most studies that report regeneration results are concerned with anion exchangers and indicate that sorption of phenolic compoundr was reversible for the conditions tested (Anderson and Hansen, 1955; Goto et al, 1986; Chanda et. al, 1985; and Polio and Kunin, 1967)6. These studies, taken together, suggest that nitrogen contrining groups such as amines (primary through quaternary), 4-vinylpyridine, and benzimidazole do not promote irreversibilities, but confirmation is needed.

Three resins with oxygen containing functional groups, XAD-7, IRC-50, and S-761 (Rohm and Haas CO.), were tested for reversibility of phenol adsorption in this work. Adsorption uptakes and degrees of regeneration were

6 Regenerations were not always carried out to the fullest possible extent in these studies and in some cases it is possible that caustic regenerant oxidized some of the sorbate. 
established in the manner detailed in section 4.1.4.1. The scintillation vials were mintained at $80^{\circ} \mathrm{C}$ and were not agitated during the adsorption contacts.

XAD-7 is an acrylic ester-divinylbenzene copolyner. The resin is usually classified as 'non-functionalizec' because it does not contain ionizable groups. The polymer matrix contains ester groups, however, and these rould interact strongly with phenol molecules. The pi-bonded oxygen is weaker Lewis bas than is a carbonyl oxygen, but could form a charge transfer complex with phenol, for example.

Figure 4.4 shows adsorption and desorption isotherms for phenol on XAD-7. The open circles represent total uptakes, while the solid bars represent the amounts of reversible adsorption. Symbols at the same phenol activity correspond to the same trial. Total and reversible adsorption amounts are within $5 \%$ of each other in every trial, indicating completely reversible adsorption within experimental error. The ester groups on XAD-7 did not promote irreversibilitias.

Crook et. al (1975) used XAD-7 to adsorb p-nitrophenol from an aqueous solution of unspecified pH at $50^{\circ} \mathrm{C}$. They recovered 97\% of tise p-nitrophenol with ethanol and found no dicernable changes in the resin capacity over three cycles. These results support the conclusion that $X A D-7$ sorbs phenolic compounds reversibly. 


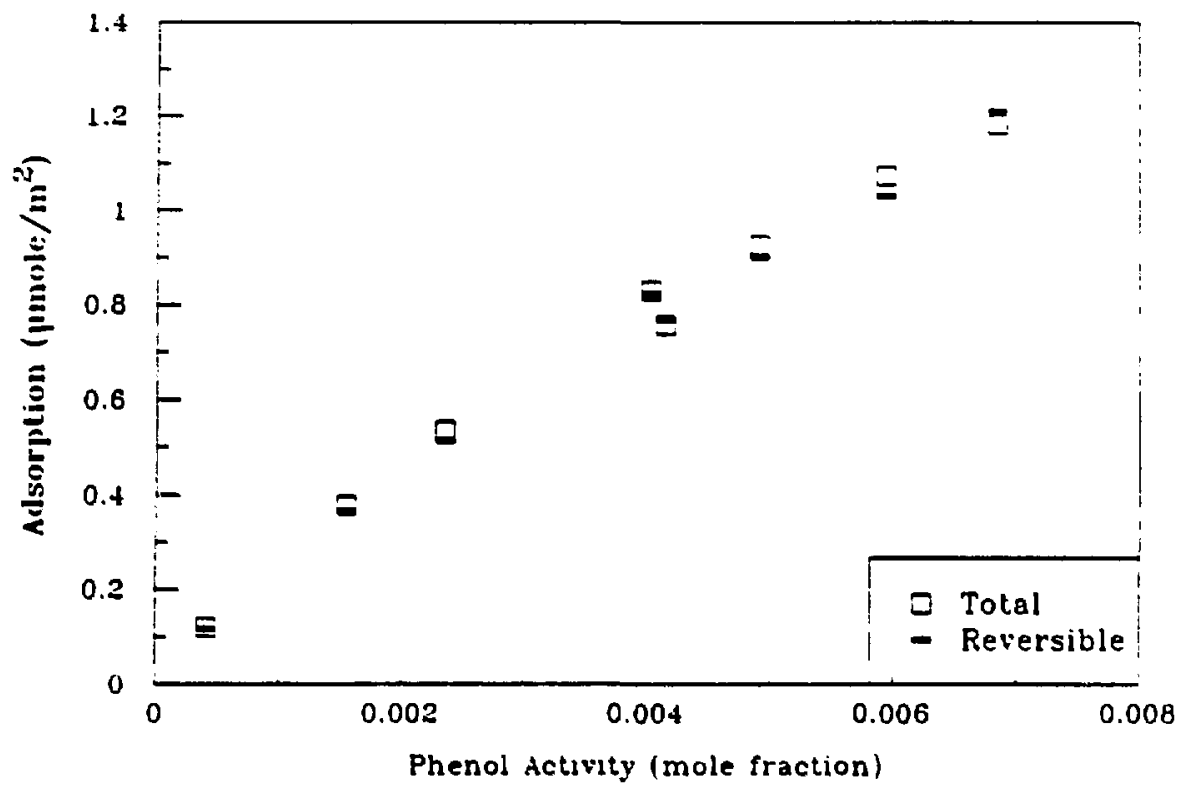

Figure 4.4 Total and Reversible Adsorption of Phenol from Water onto XAD-7. Adsorption Coatacts were for 5 Days at $80^{\circ} \mathrm{C}$. 
IRC-50 is a weak cation exchange resin containing carboxylic acid groups. The ion exchange capacity of the dry resin is $10 \mathrm{meq} / \mathrm{g}$ or slightly higher (Rohm and Haas Corp., 1977 and Waitz, 1987). The resin swells appreciably in water, so there is not a well defined surface (or surface area) for adsorptior. The resin also swells in acetone, facillitating transport of sorbate out of the particles.

Table 4.10 shows sorption and regeneration results for IRC-50. The uptake of phenol per gram dry resin is very low, and the sorption is completely reversible. The presence of carboxylic acid groups did not lead to irreversible reaction of phenol molecules.

The sorbent $5-761$ is a highly porous phenol-formaldehyde resin containing phenolic hydroxyl and methylol groups. The resin is highly crosslinked but can swell as much as $25 ;$ by volume (in caustic, Diamond Shamrock corp., 1978) The matrix is susceptible to oxidation, presumably of the phenolic hydroxyl groups.

Sorption of phenol by S-761 at $80^{\circ} \mathrm{C}$ was highly irreversible. Figure 4.5 shows curves for total, reversible, and irreversible sorption. These results, in cortrast to those reported for oxidized carbons, suggest that phenolic groups participate in irreversible reactions 
Table 4.10 Removal of Phenol from IRC-50

\begin{tabular}{ccc}
$\begin{array}{c}\text { Total Adsorption } \\
\text { ( } \mu \text { mole/g) }\end{array}$ & $\begin{array}{c}\text { Aqueous Phase } \\
\text { Phenol activity } \\
\text { (mole fraction) }\end{array}$ & $\begin{array}{c}\text { Percent } \\
\text { Regeneration }\end{array}$ \\
\hline 13.07 & 0.0046 & 99.0 \\
13.33 & 0.0045 & 100.5 \\
10.16 & 0.0040 & 105.8 \\
10.08 & 0.0040 & 105.5 \\
\hline
\end{tabular}

*Adsorption from water after 5 days of contact at $80^{\circ} \mathrm{C}$.

- Regeneration was accomplished with Acetone. 


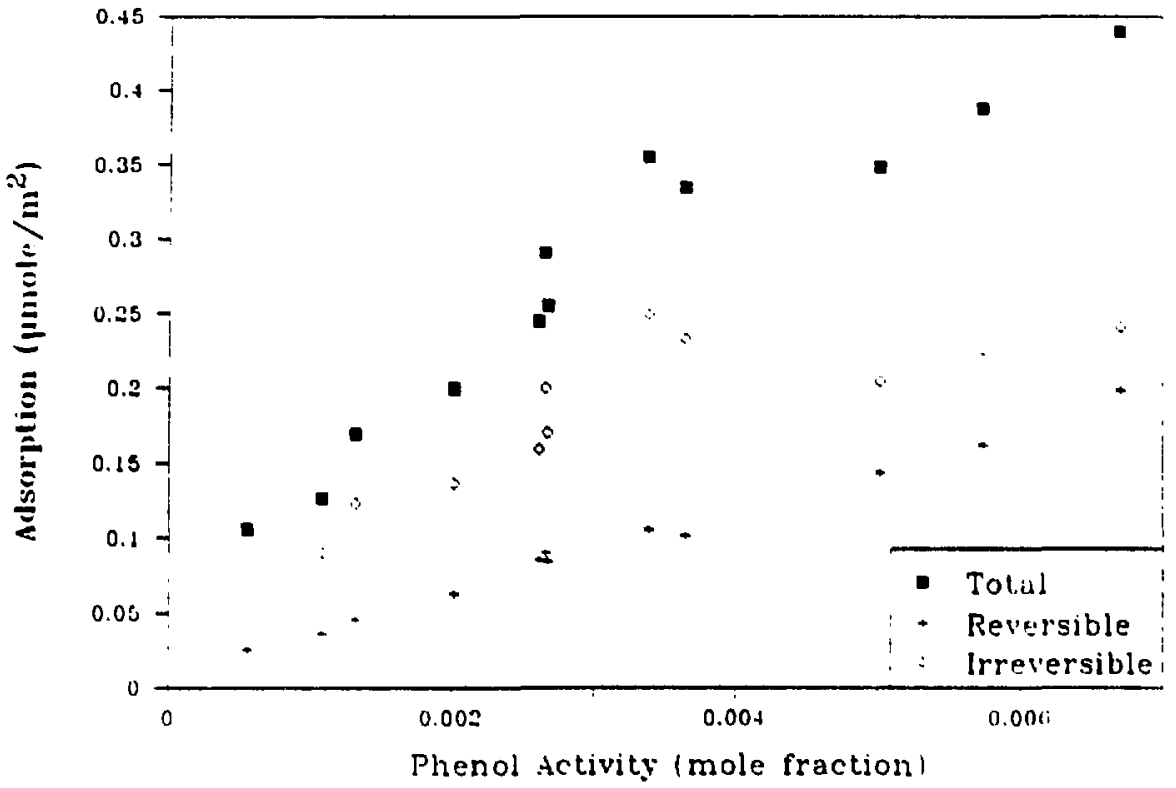

Figure 4.5 Total, Reversible and Irreversible Adsorption of Phenol from Water onto S-761. Adsorption Contacts were for 5 Days at 80. C. 
with phenol molecules. Phenolic compounds can couple irreversibly through an oxidation mechanism, as will be discussed in Chapter 5.

\subsubsection{Influence of Carbon Ash Contents on Irreversibla Adsorption}

As mentioned in Section 4.1.2, Mahajan et al. (1980) found decreased phenol uptakes for carbons with decreased ash contents. Carbons treated with hydrochloric acid adsorbed up to 25 les: phenol than did their parent carbons, for 48 hour contacts at ambient temperature. No attemp: was made to regenerate the carbons, but it is plausible that ash promoted irreversible adsorption and hence, higher uptakes. Consistently, Suzuki et al. (1978) found that thermal regeneration of phenol was less facile from a carbon with a high ash content, than from a carbon with less ash. Experimental results reported in this section indicate that ash can promote irreversible adsorption, but also that irreversible adsorption can occur on carbonaceous materials of low ash contents.

In one experiment, samples of columbia carbon were treated with aqueous solutions of potassium permanganate. one sample was immersed in $0.32 \mathrm{M} \mathrm{KMnO}_{4}$ for 26 hours. The liquid was decanted several times during the treatment period, and was replaced each time by fresh solution. A second sample was treated with a saturated solution of 
$\mathrm{KMnO}_{4}$ in the same manner. Both samples were extracted with methanol and with water in a soxhlet apparatus and then dried before further use.

Interactions of $\mathrm{KMnO}_{4}$ with carbonaceous materials are not well understood (Puri, 1970). Banerjee et al. (1962) found that alkaline and acidic solutions of permanganate reacted vigorously with coal, but did not produce carboxyl groups (as do most strong oxidizing solutions). The investigators did not identify the oxidation products. Garten and Weiss (1957b) suggested that permangante may form form lactones on carbons, but did not confirm this hypothesis. In this work, no attempt was made to identify the functional groups formed on columbia through the action of $\mathrm{KMnO}_{4}$.

Table 4.11 shows elemental analyses for columbia and for the samples treated with $\mathrm{KMnO}_{4}$. Included are analyses for potassium and manganese as elements in the ash. The ash contents of the treated samples are far greater than that of Columbia, due in large part to residual manganese. oxygen present as stable oxides of manganese would contribute to the measured ash, and probably accounts for much of the ash in the treated samples. oxidation of the carbon surfaces is reflected in increased oxygen contents of the treated samples. The values for oxygen are calculated by difference and do not account for oxygen in the ash. 
Table 4.11 Elemental Analysis of Columbia Treated with KMnO,

Elemental Analyses (Weight Percent)

\begin{tabular}{lccccccccc} 
Treatment & $\mathrm{C}$ & $\mathrm{H}$ & $\mathrm{N}$ & $\mathrm{S}$ & Ash & Total & $\mathrm{O}^{*}$ & $\mathrm{~K}$ & $\mathrm{Mn}^{* *}$ \\
\hline None & 93.70 & 0.18 & 0.0 & 3.31 & 0.4 & 97.59 & 2.41 & 0.03 & $<0.01$ \\
$0.32 \mathrm{M}$ & 77.83 & 0.28 & 0.07 & 1.69 & 12.5 & 92.37 & 7.63 & 0.79 & 6.64 \\
$\mathrm{KMnO}_{4}$ & & & & & & & & & \\
$\begin{array}{l}\text { Saturated } \\
\text { KMnO }\end{array}$ & 65.71 & 0.43 & 0.10 & 0.53 & 26.8 & 93.57 & 6.43 & 2.41 & 14.2 \\
\hline
\end{tabular}

- Determined by difference and not included in the total.

"Component of ash. 
Nitrogen BET surface areas for the two treated carbons are listed in Table 4.12. The oxidative treatment lowered the surface area of columbia.

Batch adsorption/regeneration trials were run for the $\mathrm{KMnO}_{4}$ treated carbons according to the procedures described in connection with Table 4.6. Table 4.13 summarizes the results. The created carbons adsorbed more phenol than did Columbia, despite their higher oxygen contents. Increases in irreversible adsorption were greater than increases in total adsorption, and the regenerabilities of the treated carbons were very low. These results show that ash containing a transition metal can promote irreversible adsorption.

Pretreated columbia has a low ash content compared to other vendor-supplied carbons (see Table 4.1). As shown in Table 4.14, the ash contains virtually no manganese, but does contain iron and small amounts of copper and cobalt. Periodic Table row four elements are commonly present in naturally occuring organic materials and therefore, should be common to vendor-supplied carbons. These row 4 metals can act as catalysis and can participate in oxidation-reduction reactions. Given these facts, it is reasonable to suspect that ash in many carbons could promote the irreversible adsorption of phenolic compounds. 
Table 4.12 Surface Areas for Columbia Treated with $\mathrm{KMnO}_{4}$

\begin{tabular}{|c|c|}
\hline Treatment & $\begin{array}{c}\mathrm{N}_{2} \text { BET Surface Areas } \\
\left(\mathrm{m}^{2} / \mathrm{gdc}\right)\end{array}$ \\
\hline None & 900 \\
\hline $\begin{array}{l}0.32 \mathrm{M}^{2} \\
\mathrm{KMnO}_{4}\end{array}$ & 830 \\
\hline $\begin{array}{l}\text { Saturated } \\
\mathrm{KMnO}_{4}\end{array}$ & 675 \\
\hline
\end{tabular}

Table 4.13

Comparative Regenerability of Columbia Treated with KMnO, in Various Ways

\begin{tabular}{lccccc} 
& \multicolumn{4}{c}{ Adsorption $\left(\mu \mathrm{mole} / \mathrm{m}^{2}\right)$} & \\
\cline { 2 - 5 } Treatment & Total & Total & Irrev. & Irrev. & $\begin{array}{c}\text { Percent } \\
\text { Regeneration }\end{array}$ \\
\hline None & $2.51 \pm .03$ & $\cdots$ & $1.62 \pm .04$ & $\ldots .$. & $35.7 \pm 1.2$ \\
$0.32 \mathrm{M} \mathrm{KMnO}_{4}$ & $2.69 \pm .08$ & 0.19 & $2.08 \pm .08$ & 0.46 & $22.8 \pm 0.8$ \\
Saturated & $2.81 \pm .12$ & 0.30 & $2.38 \pm .05$ & 0.76 & $15.3 \pm 1.8$ \\
KMnO, & & & & & \\
\hline
\end{tabular}

"Irreversible

Table 4.14 Ash Composition of Witco Columbia

\begin{tabular}{cc} 
Element & $\begin{array}{c}\text { Wcight Percent of } \\
\text { Columbia }\end{array}$ \\
\hline $\mathrm{Fe}$ & 0.159 \\
$\mathrm{Cr}$ & $<0.01$ \\
$\mathrm{Cu}$ & 0.013 \\
$\mathrm{Co}$ & 0.031 \\
$\mathrm{Mn}$ & $<0.01$ \\
$\mathrm{~K}$ & 0.026 \\
$\mathrm{Na}$ & 0.195 \\
Total & 0.424 \\
\hline
\end{tabular}


While mineral ash can promote irreversibilites, irreversible adsorption also occurs on materials with little or no ash. An example is $5-761$, the phenol-formaldehyde resin discussed in section 4.1.4.5. Two further examples are Rohm and Haas Ambersorbs XE-340 and $\mathrm{XE}-348$, carbonaceous adsorbents that have been used to remove phenolics from water (Chang and Savage, 1981; Mahajan et al., 1980; and Polysciences Inc., 1986).

$X E-340$ and $X E-348$ are pyrolized polymeric adsorbents. They are derived from a polystyrene-divinylbenzene copolymer that is sulfonated and then pyrolyzed to create a carbonaceous residue (Neeley, 1977, 1981). XE-348 is pyrolyzed to a greater extent than is $X E-340$ and consequently, has a much higher skeletal density (1.95 vs $1.34 \mathrm{~g} / \mathrm{cm}^{3}$. Polysciences Inc., 1986).

3 Elemental analyses for $X E-340$ and $X E-3 \div 8$ are given in Table 4.15. Neither adsorbent contains a significant amount of ash. $\mathrm{XE}-348$ has higher carbon to hydrogen and sulfur to oxygen ratios than does $X E-340$, again reflecting a greter extent of pyrolysis. Sulfur should be in a lower oxidation state in XE-348 than in XE-340 (see also Neeley, 1981).

Table 4.16 shows the results of adsorption/regeneration trials carried out in the usual way. XE-340 is hydrophobic and was prewet by contact with methanol, followed by water washing. XE-348 adsorbed much more phenol than did XE-340, 
Table 4.15 Elemental Analysis of XE-340 and XE-348*

Elemental Analyses (Weight Percent)

\begin{tabular}{lccccccc} 
Adsorbent & $\mathrm{C}$ & $\mathrm{H}$ & $\mathrm{N}$ & $\mathrm{S}$ & $\mathrm{O}$ & Ash & Total \\
\hline $\mathrm{XE}-340$ & 87.97 & 3.70 & 0.17 & 3.67 & 2.11 & $<0.1$ & 97.72 \\
XE-348 & 93.32 & 0.67 & 0.13 & 3.71 & 0.68 & 0.1 & 98.61 \\
\hline
\end{tabular}

(Munson. 1985)

Table 4.16 Comparative Regenerabillty of XE-340 and XE-348

\begin{tabular}{lccc} 
& \multicolumn{2}{c}{ Adsorption $\left(\mu \mathrm{mole} / \mathrm{m}^{2}\right)$} & $\begin{array}{c}\text { Percent } \\
\text { Adsorbent }\end{array}$ \\
\cline { 2 - 3 } & Total & Irrev. & Regeneration \\
\hline XE-340 & $1.48 \pm .07$ & $0.23 \pm .00$ & $84.4 \pm 0.6$ \\
XE-348 & $3.34 \pm .04$ & $2.46 \pm .01$ & $26.2 \pm 1.1$ \\
\hline
\end{tabular}

-Irreversible 
but the difference was due to irreversible adsorption. The reason for the lower regenerability of XE-348 is not apparent (see also section 4.1 .6 ), but it cannot be attributed to reactions with ash.

In summary, ash containing manganese promoted irreversible adsorption of phenol, and it is reasonable to suspect that other transition metals in ash could have the same fect. Irreversible adsorption can occur in the absence of such metals, however.

\subsubsection{Influence of Unpaired Electrons on Irreversible Adsorption}

Unpaired electrons form in activated carbons as a result of carbonization of organic materials (Ingram et al., 1954; Ingram, 1955). These electrons are thought to be localized at the edges of graphitic layers or at defects within the layers, and they are thought to be very reactive (Coughlin and Tan, 1968; Puri, 1970). Reactions of unpaired electrons with heteroatoms result in surface functional groups, and it is therefore thought that unpaired electrons play a role in chemisorption (Coughlin and Tan, 1968; Puri, 1970). It seems reasonable to postulate, therefore, that irreversible adsorptinn of phenolic compounds could be due to chemlcal reactions involving unpaired electrons. This postulate is scrutiniuzed in this section. 
Magne and walker (1986) found that a portion of the phenol adsorbed onto various activated carbons could not be recovered as phenol. They suggested that phenol was chemisorbed onto sites of the "active surface areas" of the carbons. These sites were defined as carbon atoms at the edges of basal planes. The investigators based their suggestion on the observation that carbon oxidized with $\mathrm{O}_{2}(\mathrm{~g})$ chemisorbed less phenol than did the parent carbon. They did not propose a mechanism for the chemisorption, nor did they mention unpaired electrons, but they did suggest that treatment of carbon with $\mathrm{O}_{2}(\mathrm{~g})$ would occupy the active surface area, theraby inhibiting chemisorption of phenol.

Harker et al. (1961) used electron paramagnetic resonance (EPR) to study the effects of oxidation with $\mathrm{O}_{2}(g)$ on the concentration of unpaired electrons in activated carbon. They found that the intensity of the EPR signal for a carbon decreased due to a nonactivated reaction with $\mathrm{O}_{2}(g)$ at room temperature. The original signal intensity was restored after the carbon was heated under vacuum. The investigators also found that an activated "chemisorption" of oxygen occured at $400^{\circ} \mathrm{C}$. The EPR signal decreased as the reaction proceeded, and could not be restored by heating the carbon urder vacuum. These results, together with the observations of Magne and Walker, are consistent with the postulate stated earlier. 
In contrast, further studies by Harknr et al. (1966) indicated that the number of unpaired electrons on a carbon is not directly related to its reactivity with $\mathrm{O}_{2}(\mathrm{~g})$. The investigators did find a tionship between the rumber of unpaired electrons and the ability of a c-rbon to adsorb No $(g)$, however. The researchers surce th: nitric oxide could act as an electron acceptu. an? form cheige-transfer complexes with unpaired electrons delocalized (resonance stabalized) in basal planes. The bondin: would be weak and reversible. These results do not preclude irreversible reactions of phenolic compounds with unpaired electrons, but they do indicate that the electrons need not be highly reactive.

There is also evidence that oxidetion increases unpaired electron concentrations. Antonowicz (1962, 1964) found that unpaired electrons resulted from chemical attack on carbons by $\mathrm{O}_{2}(g)$ at ambient and at elevated temperatures. Reiss and Donnet (1964) found that oxidation of carbon blacks with nitric acid increased the number of free radicals in arioxy-form. Considering the observations that oxidation with $\mathrm{O}_{2}(g)$ or with $\mathrm{HNO}_{3}$ increases the regenerability of carbons (Magne and walker, 1986 and Section 4.1 .4 .2 of this work), this is evidence against the postulate being examined. 
In this work, an experiment was carried out to determine whether the relative concentrations of free electrons on adsorbents correlate with their relative tendencies to adsorb phenol irreversibly. The sorbents tested were XAD-4, XE-340, and XE-348. Physiochemical properties and adsorption characteristics of these solids were discussed earlier (see sections 4.1 .1 and 4.1.5).

Concentrations of unpaired electrons were measured by Dr. Paul skerker7 using a Bruker ER200D-SRC EPR spectrometer. A microwave power of 10 or $11 \mathrm{~dB}$, a modulation amplitude of $1.0 \mathrm{G}$, and a scan range of $100 \mathrm{G}$ were used. An amorphous hydrogenated silicon standard was used as a reference. The standard contained $5 \times 1016$ spins $/ \mathrm{Cm}^{3}$. Adsorbent samples were placed in glass NMR tubes, and dried for 48 hrs under vacuum before the analyses.

As discussed in Section 4.1.5, XE-348 is more carbonized than is XE-340. Since carbonization can generate unpaired electrons, it might be expected that the relative concentrations of these electrons follow the order $X E-348>X E-340>X A D-4^{8}$. As shown in Table $4.17, E P R$ measurements do not support that ordering. The

7 Dr. Skerker carried out EPR analyses in the Berkeley Laboratory of Douglas Clark. Dr. Skerker received his Ph. D. in Chemical Engineering at Cornell University in 1988 .

a XAD-4 is not carbonized, but was included in this experiment because polystyrene-divinylbenzene is a 'starting material' for the production of XE-340 and $X E-348$. 
Table 4.17 Unpaired Electrons and Comparative Regenerability of XAD-4, $\mathrm{XE}-340$, and $\mathrm{XE}-348$

\begin{tabular}{lcccc} 
& & \multicolumn{2}{c}{ Adsorption $\left(\mu \mathrm{mole} / \mathrm{m}^{2}\right)$} & \\
\cline { 3 - 4 } Adsorben: & $\begin{array}{c}\text { Unpaired } \\
\text { Electrons } \\
\text { spin } / \mathrm{m}^{2}\end{array}$ & Total & Irrev. $^{*}$ & $\begin{array}{c}\text { Percent } \\
\text { Regeneration }\end{array}$ \\
\hline XAD-4 & $1.1 \times 10^{2}$ & $0.68 \pm .04$ & $0.00 \pm .01$ & $100 \pm 0.8$ \\
XE-340 & $5.0 \times 10^{6}$ & $1.48 \pm .07$ & $0.23 \pm .00$ & $84.4 \pm 0.6$ \\
XE-348 & $7.6 \times 10^{3}$ & $3.34 \pm .04$ & $2.46 \pm .01$ & $26.2 \pm 1.1$ \\
\hline
\end{tabular}

"Irreversible 
concentration of unpaired electrons in $\mathrm{XE-340}$ is three order of magnitude greater than that in $X E-348$, and four orders of magnitude higher than that in XAD-4. Harker and coworkers (1961, 1966) found maxima in curves of unpaired electron concentration vs. carbonizing temperature or vs. weight percent carbon for coals and charcoals, so a decrease in concentration with increased carbonization is not unreasonable.

Table 4.17 also shows the results of batch adsorption/regeneration trials for the three adsorbents. The tendency to adsorb phenol irreversibly increases with degree of carbonization, but does not correlate with unpaired electron concentrations. These facts indicate that irreversible adsorption of phenol is not caused primarily by unpaired electrons in the adsorbent materials.

\subsection{Influences of Sorbate properties on Irreversible Adsorption}

Irreversible adsorption of phenolics by carbon is due to chemical reactions involving sorbate molecules. Consequently, determination of the chemical properties that influence irreversible uptakes affords insights into potential reaction mechanisms. In this section, sorbate reactivities are compared, where the term reactivity refers to the tendency of a sorbate to undergo irreversible 
adsorption. Many of the comparisons are made at equal solute activities, according to the discussion and the activity coefficient values in Appendix 2 .

\subsubsection{Nonreactive Sorbates}

Three compounds investigated in this work were completely recovered from activated carbon. Regeneration of carbons was accomplished according to the procedure described in section 4.1.4.1. Note that there was irreversible adsorption of phenol onto the same carbons under similar adsorption contact conditions (see chapters 2 and 3 for F-100 and Section 4.1 .4 for Columbia).

Adsorption of 5,5-dimethylhydantoin (5,5-dmh) from water onto $20 \times 40$ mesh F-100 was fully reversible. Adsorption contacts were for 10 days at $25^{\circ} \mathrm{C}$ with mechanical agitation. 5,5-Dmh loadings, corresponding aqueous phase concentrations, and percent recoveries with methanol are listed in Table 4.18. Regeneration was complete to within $6 f$ in each case.

Adsorption was also completely reversible for nitrobenzene and for methoxybenzene (anisole) on Columbia carbon. The adsorption conditions were those detailed in Section 4.1.4.1 except that a variety of carbon weights, and $20 \mathrm{ml}$ aliquots of a variety of aqueous solutions were 
Table 4.18 Removal of 5,5-Dimethylhydantoin From F-100 with Methanol

\begin{tabular}{ccc}
$\begin{array}{c}\text { Total Adsorption } \\
\left(\mu \text { mole } / \mathrm{m}^{2}\right)\end{array}$ & $\begin{array}{c}\text { Aqueous Phase } \\
5,5-\text { Dmh Concentration } \\
(\text { mmole/I) }\end{array}$ & $\begin{array}{c}\text { Percent } \\
\text { Regeneration }\end{array}$ \\
\hline 0.730 & 3.22 & 97.4 \\
0.638 & 2.08 & 94.3 \\
0.539 & 1.45 & 94.3 \\
\hline
\end{tabular}

-Adsorption from water after 10 days of contact at $25 \circ \mathrm{C}$. 
used to establish the isotherms 9 . Carbon weights varied from 0.0027 to $0.034 \mathrm{~g}$ for the nitrobenzene trials and from 0.0027 to $0.27 \mathrm{~g}$ for the anisole trials. Initial solution concentrations were $\leq 7.35 \times 10^{-3} \mathrm{M}$ for nitrobenzene and $\leq$ $9.22 \times 10^{-3} \mathrm{M}$ for anisole. Figures $4.6 \mathrm{ab}$ show the adsorption and the regeneration results. Although there is considerable scatter (particularly at high concentrations of nitrobenzene), the data indicate that the benzenes were fully recovered from columbia.

There are plateaus in the 'total adsorption' isotherms in Figures 4.6ab10. The uptakes in the plateau regions indicate that each benzene molecule occupies between 0.41 and 0.45 square nanometers of the surface. This surface area corresponds roughly to the area of a molecule oriented parallel to the surface (Chang et al., 1980; Coughlin et al., 1968). In this orientation, nonpolar forces acting over the entire sorbate nucleus would be the dominant interaction (Giles et al., 1960; Coughlin and Ezra, 1968).

These benzenes fall close to the border between Group II and Group III compounds in the plot of aromaticity vs. boiling point developed by suzuki, et al. (1978). This

9 These benzenes are quite volatile, and care was taken to minimize vapor losses. Each aqueous phase was sampled by puncturing a small hole in the cap of a vial and inserting a syringe needle. Centrifuge times were kept as low as possible for good phase separation.

20 The plateau for nitrobenzene is definite, but data at higher activities are needed to ensure that anisole has reached a plateau. Compare the activity scales in the figures. 


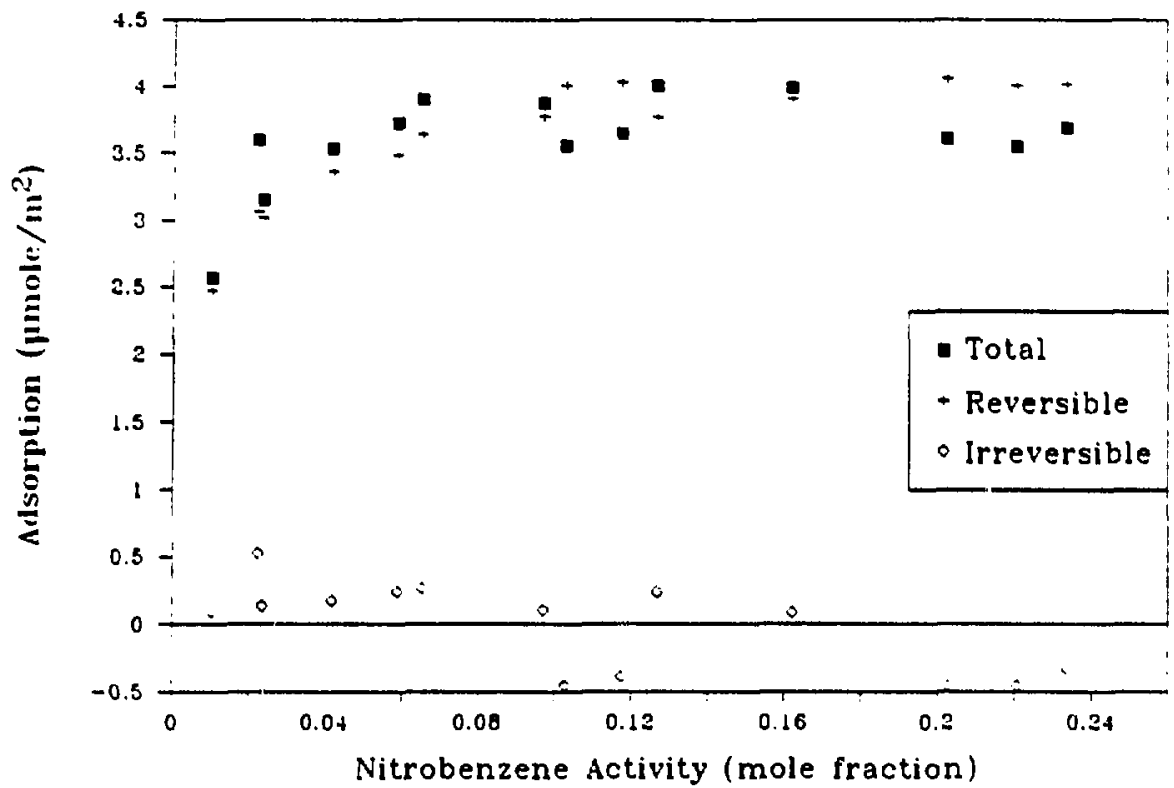

Figure 4.6a Total, Reversible and Irreversible Adsorption of Nitrobenzene from Water onto Columbia Carbon. Adsorption Contacts were for 5 Days at $80^{\circ} \mathrm{C}$. 


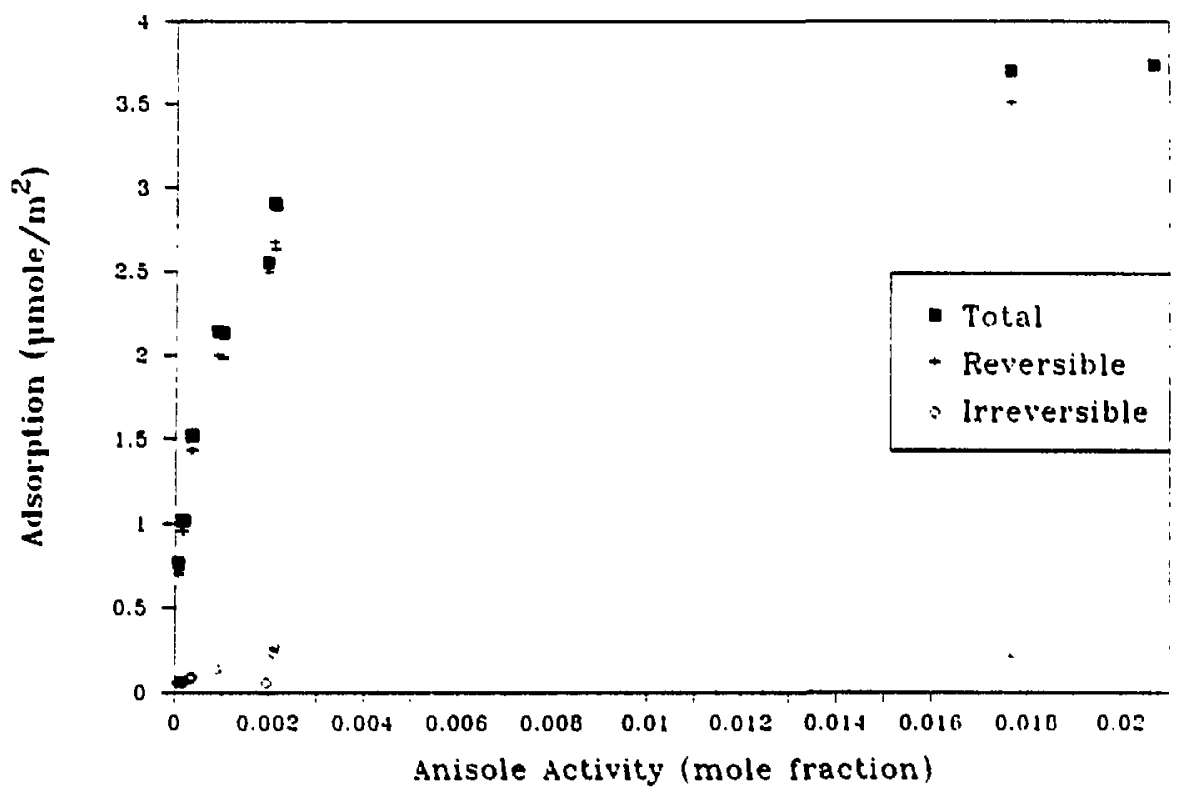

Figure 4.6b Total, Reversible and Irreversible Adsorption of Methoxybenzene from Water onto Columbia Carbon. Adsorption Contacts were for 5 Days at $80^{\circ} \mathrm{C}$. 
fact, together with Figures $4.6 \mathrm{ab}$, shows that the correlation of Suzuki, et al., does not fully apply to the definition of irreversible adsorption developed in this work. It may be expected that compounds that can be completely desorbed thermally can be corpletely desorbed with acetone, but the converse may not apply. The volatilities of sorbates are critical to the results of thermal regenerations, but should not directly affect solvent regenerations. Therefore, the boiling point of a sorbate is not expected to influence its tendency to be irreversibly adsorbed in the present work.

Aromaticity does influence irreversible adsorption, but only under certain circumstances. Phenol is irreversibly adsorbed, for example, under conditions where benzenes and/or normal alcohols are notll. Neither the hydroxy group on a normal alcohol nor the aromatic ring of a benzene is reactive, but hydroxybenzenes are reactive.

\subsubsection{Dihydroxybenzenes}

Dihydroxybenzenes are even more reactive than phenol. Figure $2.2 \mathrm{~b}$ showed that resorcinol has more affinity for Filtrasorb-100 than does phenol, despite the fact that resorcinol has a lower aqueous phase activity coefficient $\left(0.61\right.$ vs 63.53 at $\left.25^{\circ} \mathrm{C}\right)$. Further, up to 618 of the adsorption of hydroquinone onto columbia was irreversible

11 See Table 1.2 and the accompanying discussion. 
for 5 day contacts at $25^{\circ} \mathrm{C}$ (see section 4.3). Under similar conditions, phenol should be much less reactive (see section 3.3.2 for example). These facts show that hydroxyl groups enhance the reactivity of aromatic sorbates.

\subsubsection{Monohydroxybenzenez}

Substituents to the aromatic rings of monohydroxybenzenes influence their tendencies to undergo irreversibie adsorption. Total and reversible uptakes onto columbia carbon were measured for several

monohydroxybenzenes. The adsorption conditions were those detailed in section 4.1.4.1, except that a variety of carbon weights and a variety of single-solute aqueous solutions were used to establish the adsorption curves. Under these conditions, reaction kinetics may influence adsorption amounts, but the curves give an indication of the relative reactivities of the sorbates.

Figure 4.7 shows total, reversible, and irreversible uptakes of phenol. The data from trials carried out at a number of different times are collected in the figure, and the scatter reflects variations in "controlled" experimental variables (e.g., HPLC and GC calibrations and temperature control which improved in later trials). 


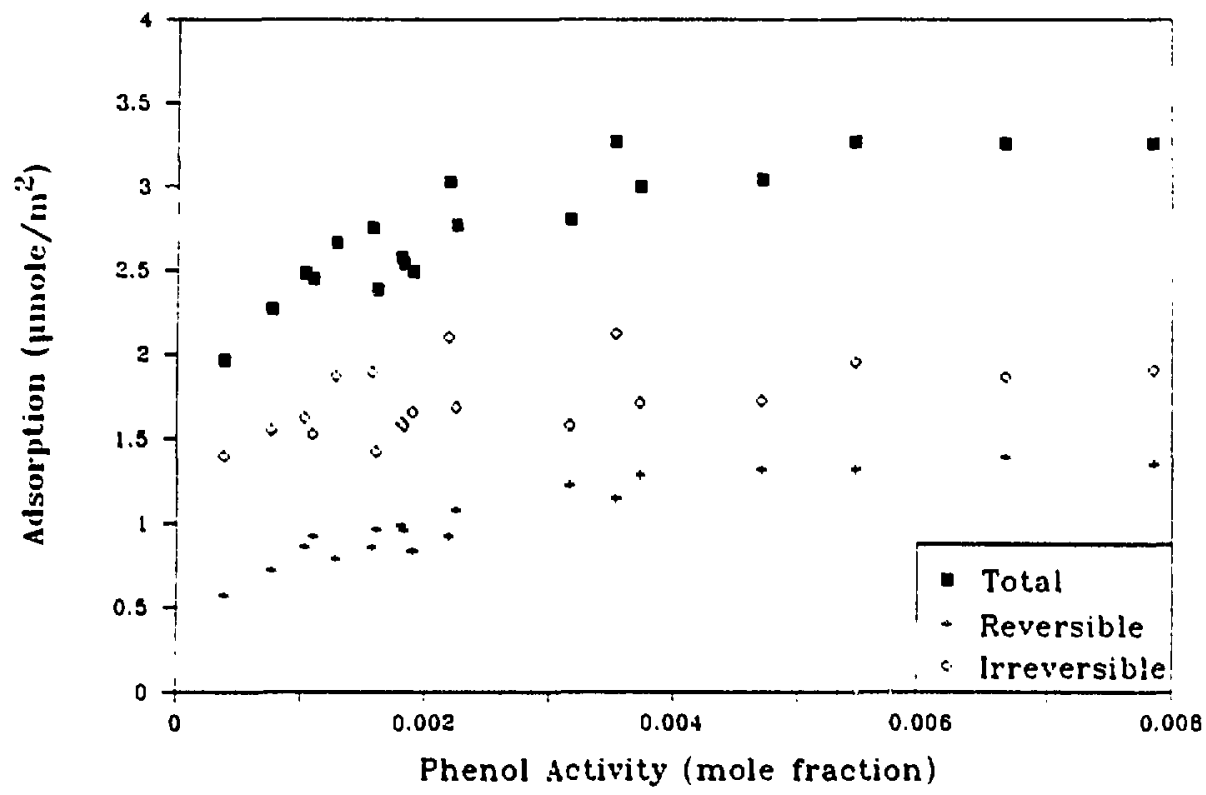

Figure 4.7 Total, Reversible and Irreversible Adsorption of Phenol rrom Water onto Columbia Carbon. Adsorption Contacts were for 5 Days at $80^{\circ} \mathrm{C}$. 
Initial solution concentrations of $1.28 \times 10^{-2}, 6.38 \times$ $10^{-3}$, and $6.27 \times 10^{-3} \mathrm{M}$ were used, and carbon weights varied from 0.001 to $0.075 \mathrm{~g}$.

Total, reversible, and irreversible adsorption of phenol all increase with increasing activity of phenol in solution. Reversible adsorption is higher at higher activities, despite increased coverage of the surface by irreversibly held sorbate. This is due to an increased equilibrium driving force for physisorption, and perhaps to the ability of phenol molecules to adsorb onto irreversibly adsorbed molecules. Although it appears that all three types of adsorption reach plateaus at higher activities, it will be shown that much higher uptakes (all three types) are achieved at still higher activities (see section 4.3).

Figure 4.8 shows the results of adsorption/regeneration trials with 2,4-dimethylphenol (2,4-dmp). The initial solution concentration was $8.42 \times 10^{-3} \mathrm{M}$, and the carbon weights varied between 0.002 and $0.06 \mathrm{~g}$. At a given activity, there is somewhat more total and irreversible adsorption, and somewhat less reversible adsorption of 2,4-dmp than of phenol. These facts indicate not only that 2,4-dmp is more reactive than phenol, but also that irreversible adsorption decreases the capacity of Columbia for reversible adsorption. 


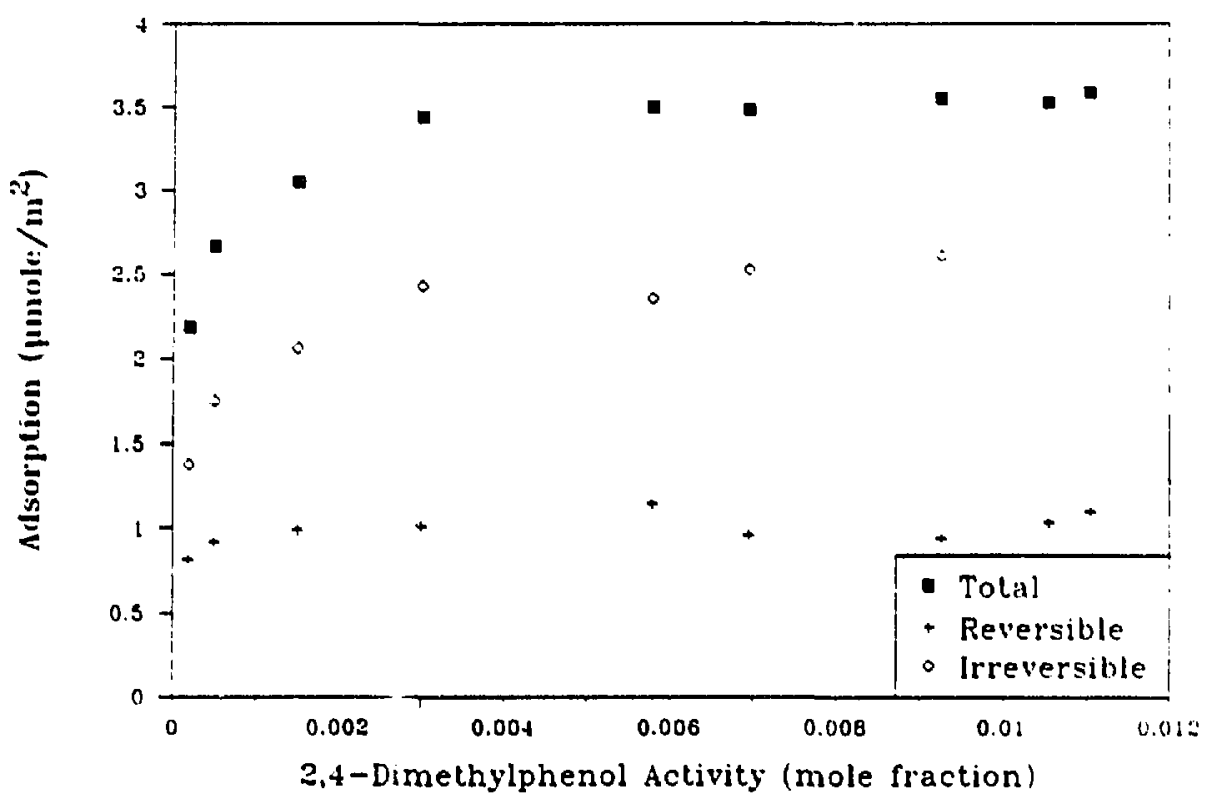

Figure 4.8 Total, Reversible and Irreversible Adsorption of 2,4-Dimethylphenol from Water onto Columbia Carbon. Adsorption Contacts were for 5 Days at $80^{\circ} \mathrm{C}$. 
Total, reversible, and irreversible uptakes of p-chlorophenol are illustrated in Figure 4.9. Initial solution concentrations of $1.56 \times 10^{-3}$ and $7.79 \times 10^{-4} \mathrm{M}$ and carbon weights between 0.001 and $0.021 \mathrm{~g}$ were used to establish the data. The adsorption amounts are similar to those for 2,4-dimethylphenol over the same range of activities.

Adsorption amounts for p-methoxyphenol (pmp) are shown in Figure 4.10. The initial solution concentration was $6.45 \times 10^{-3} \mathrm{M} \mathrm{pmp}$, and carbon weights varied between 0.002 and $0.06 \mathrm{~g}$. A slight yellow tinge developed in the aqueous phases of trials with lower carbon weights and in a blank trial (6.45 $\times 10^{-3} \mathrm{M} \mathrm{pmp}$ and no carbon). The concentration change in the blank trial was negligible, so no adjustments were made to the mass balances.

At corresponding activities, totai and irreversible uptakes are greater and reversible uptakes are lower for pmp than for phenol. These facts indicate that the methoxy-group promotes irreversible adsorption. Since methoxybenzene is not reactive, the methoxy-group itseif is probably not reactive. That is, it is reasonable to speculate that the methoxy-group activates the phenol, but does not particlpate directly in a chemical reaction.

The shapes of the three curves in Figure 4.10 deserve comment. The curves for total and irreversible adsorption seem to have inflections at activity ca. 0.004), but the 


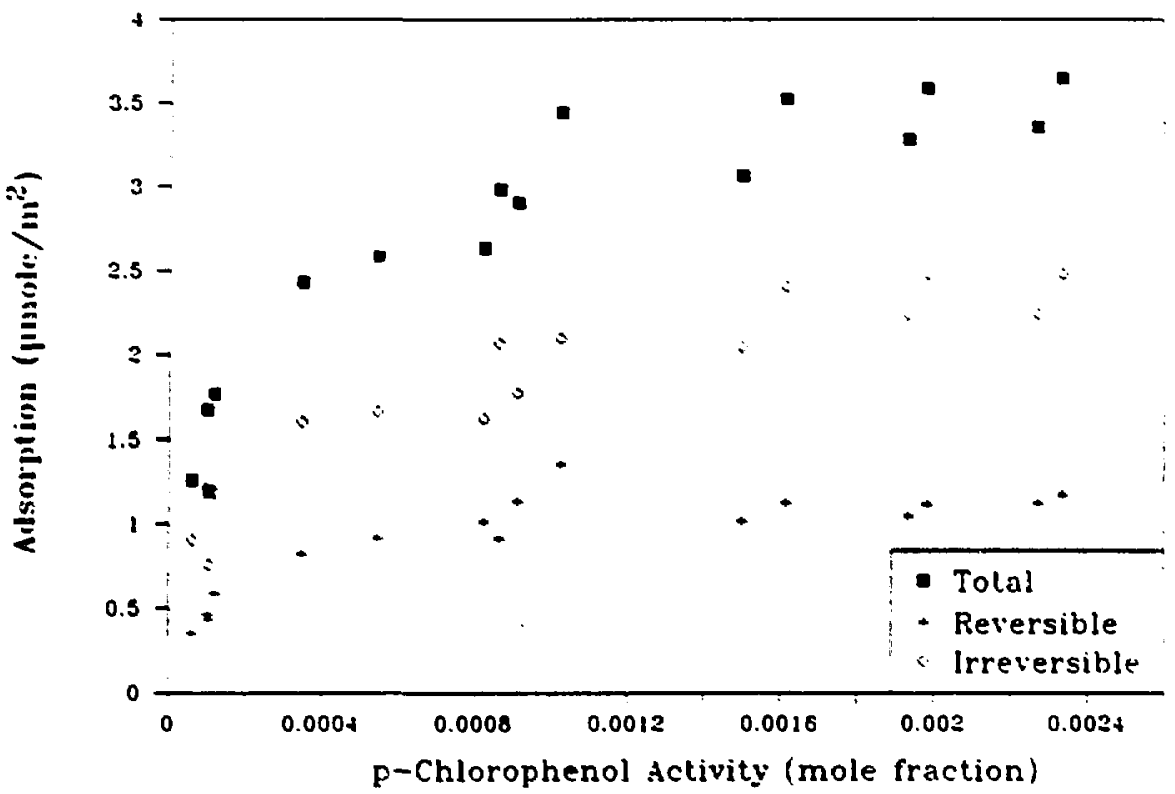

Figure 4.9 Total, Reversible and Irreversible Adsorption of p-Chlorophenol from Water onto Columbia Carboa. Adsorption Contacts were for 5 Days at $80^{\circ} \mathrm{C}$. 


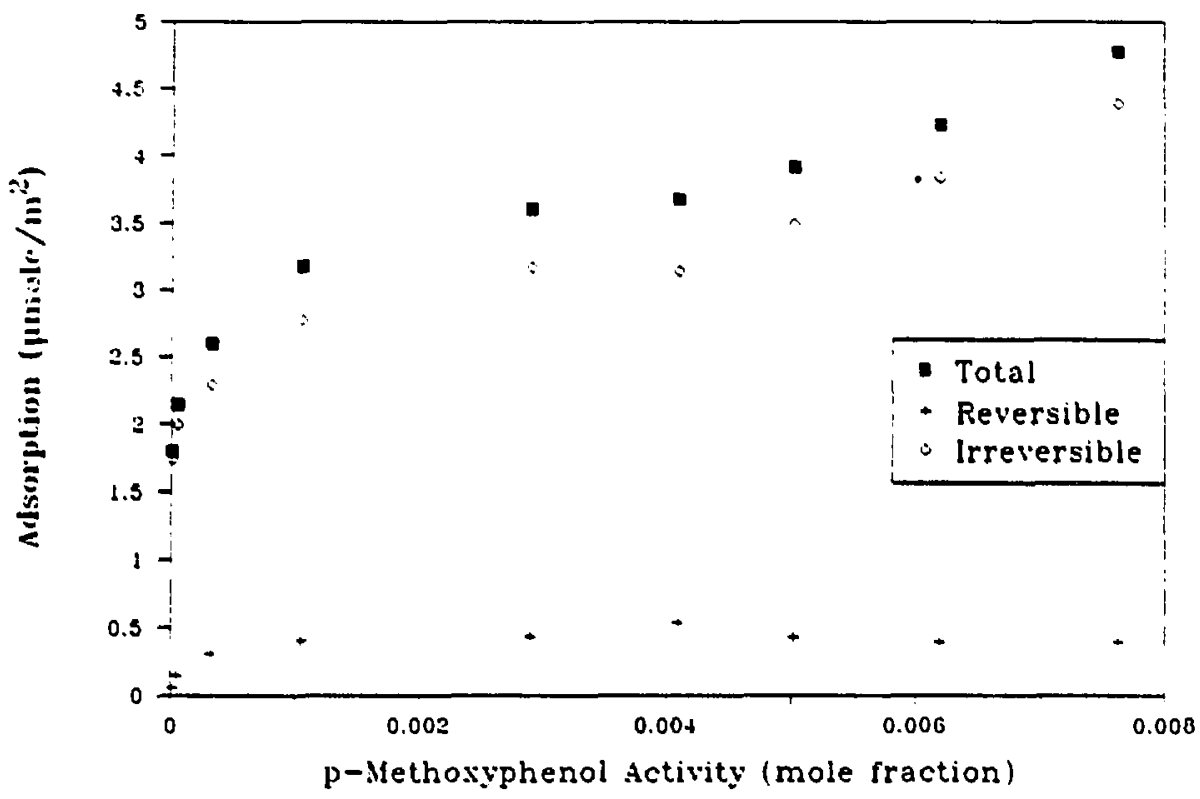

Figure 4.10 Total, Reversible and Irreversible Adsorption of p-Methoxyphenol from Water onto Columbia Carbon. Adsorption Contacts were for 5 Days al $80^{\circ} \mathrm{C}$. 
curve for reversible adsorption does not. Several investigators have observed inflections in isotherms for adsorption of phenols onto carbons (Coughlin and Ezra, 1968; Mattson et al., 1969; see also the discussion of this topic by Mattson and Mark, 1971). Generally, those isotherms were established under conditions that should not have led to large amounts of irreversible adsorption. Correspondingly, the inflections were attributed to reorientation of weakly adsorbed molecules. The absence of an inflection in the curve for reversible adsorption in Figure 4.10 is inconsistent with that interpretation. The inflection in the curve for total adsorption may possibly be due to experimental inaccuracies, and the inflection in the curve for irreversible adsorption would then arise because the irreversible uptakes were calculated by difference.

More significant are the magnitudes of total and irreversible adsorption at the highest activities in Figure 4.10. The uptakes are greater than the plateau values in Figures $4.6 \mathrm{ab}$, which can be explained by one or more of the following: First, sorbed p-methoxyphenol molecules may be oriented perpendicular to the surface, so that they take up less area than the substituted benzenes (assumed to lie flat on the surface). Second, irreversibly adsorbed molecules may occupy less area than do molecules that are 
reversibly adsorbed. Third, multiple adsorbed layers could form. In any case, interactions between adsorbed molecules are important at relativly high activities.

Results for adsorption/regeneration trials with p-hydroxybenzaldehyde (phb) are summarized in Figure 4.11. Initial solution concentrations of $9.84 \times 10^{-3}$ and $4.92 \times$ $10^{-3} \mathrm{M}$ were used in the adsorption trials, as were carbon weights from 0.012 to $0.06 \mathrm{~g}$. p-Hydroxybenzaldehyde has much less tendency to adsorb irreversibly than does phenol.

Surprisingly, there is less total adsorption of p-hydroxybenzaldehyde than of anisole at a given solute activity. This is probably due to inaccurate estimation of one or both of the infinite dilution activity coefficients. The activity coefficient for phb is especially suspect because it is based solely on the solubility at $30.5^{\circ} \mathrm{C}$ (see Appendix 2). For equal solute activities, there should probably be slightly more total adsorption of phb than of anisole, because the former undergoes irreversible as well as reversible adsorption.

The results for p-nitrophenol (pnp) are in line with this reasoning. p-Nitrophenol also has little tendency to adsorb irreversibly, as shown in Figure 4.12. Initial solution concentrations of $2.16 \times 10^{-2}, 1.08 \times 10^{-2}$, and $4.32 \times 10^{-3} \mathrm{M}$ pnp and carbon weights of 0.004 to $0.12 \mathrm{~g}$ were used in the adsorption trials. Comparison of Figures 4.10 and 4.6 a shows that at a given activity, slightly more 


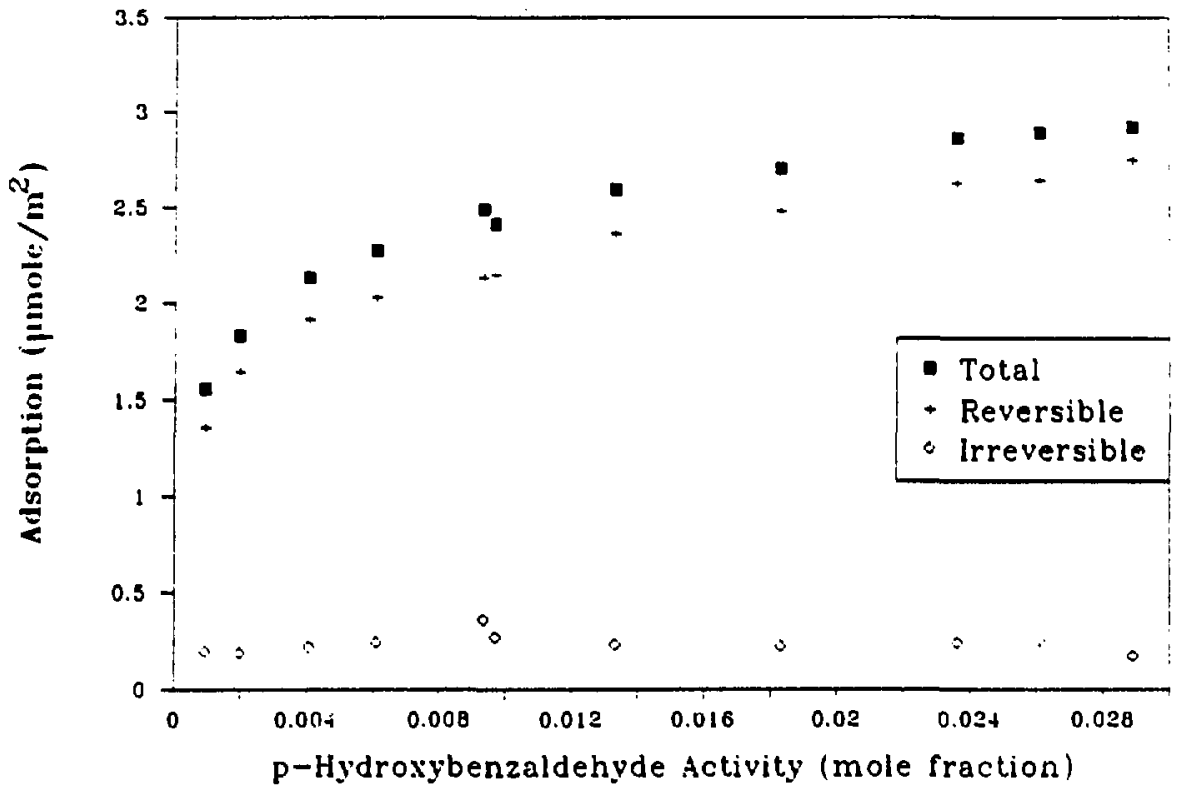

Figure 4.1! Total, Reversible and Irreversible Adsorption of p-Hydroxybenzaldehyde from Water onto Columbia Carbon. Adsorption Contacts were for 5 Days at $80^{\circ} \mathrm{C}$. 


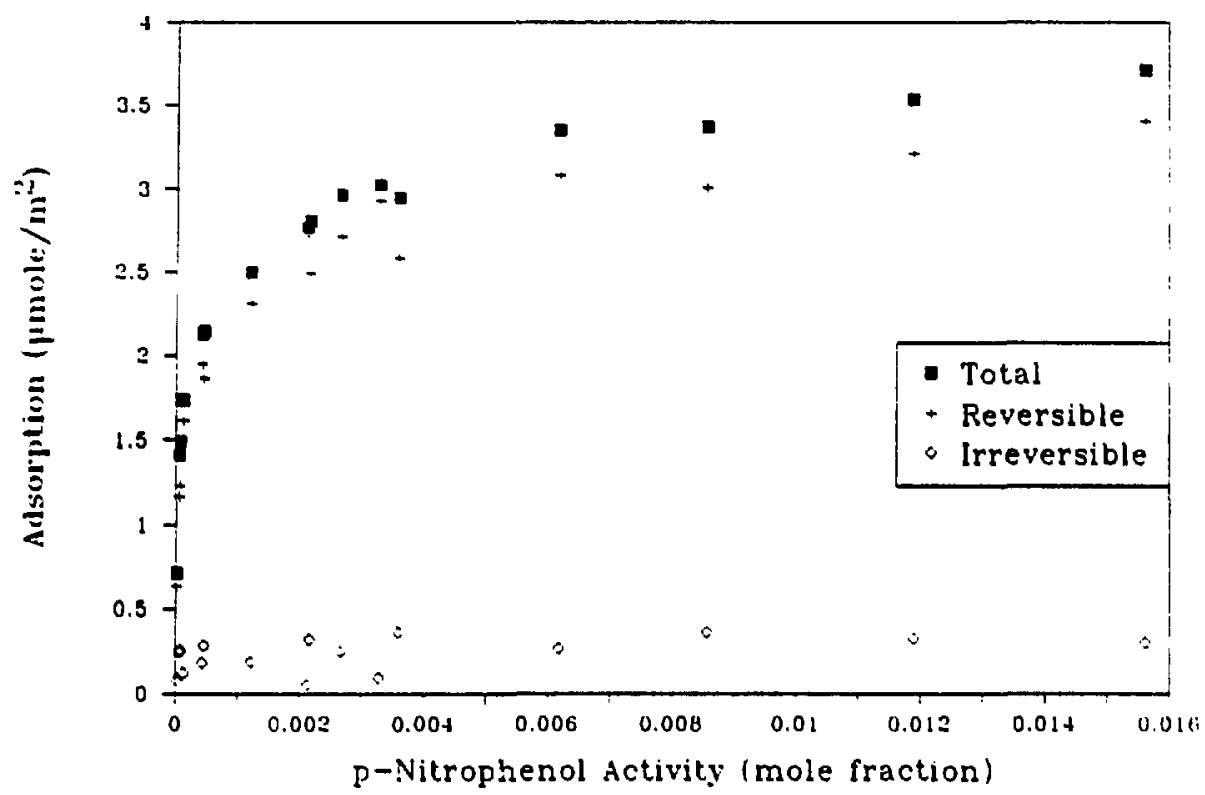

Figure 4.12 Total, Reversible and Irreversible Adsorption of p-Nitrophenol from Water onto Columbia Carbon. Adsorption Contacts were for 5 Days at $80^{\circ} \mathrm{C}$. 
pnp than anisole is adsorbed. This is consistent with observations made earlier which indicate that irreversible adsorption increases the total capacity of a carbon in a single adsorption contact (see section 4.1.4.3).

\subsubsection{Aniline}

Aniline also undergoes irreversible adsorption onto columbia carbon, as is shown in Figure 4.13. The trials represented in the figure were carried out in the same manner as were those for monohydroxybenzenes. Initial solution concentrations of $2.20 \times 10^{-2} \mathrm{M}$ and $1.10 \times 10^{-2} \mathrm{M}$ and carbon weights between 0.008 and $0.05 \mathrm{~g}$ were used. Although ammonia may react with activated carbon (Mahajan et al, 2978), it is reasonable to postulate that the mechanism for irreversible adsorption is similar for aniline and for hydroxybenzenes. Indirect evidence for this postulate is given in Chapter 5 . Comparison of Figure 4.13 with Figures $4.7,4.11$, and 4.12 indicates that aniline is less reactive than phenol, but more reactive than p-hydroxybenzaldehyde and p-nitrophenol.

\subsubsection{A Relative Ordering of Renctivities}

As indicated in the discussion of Figures $4.6 \mathrm{ab}$ through 4.13, the substituted benzenes have markedly different reactivities as sorbates on activated carbon. Since the reaction mechanisms are unknown, however, and since several physiochemical processes occur during adsorption, the data 


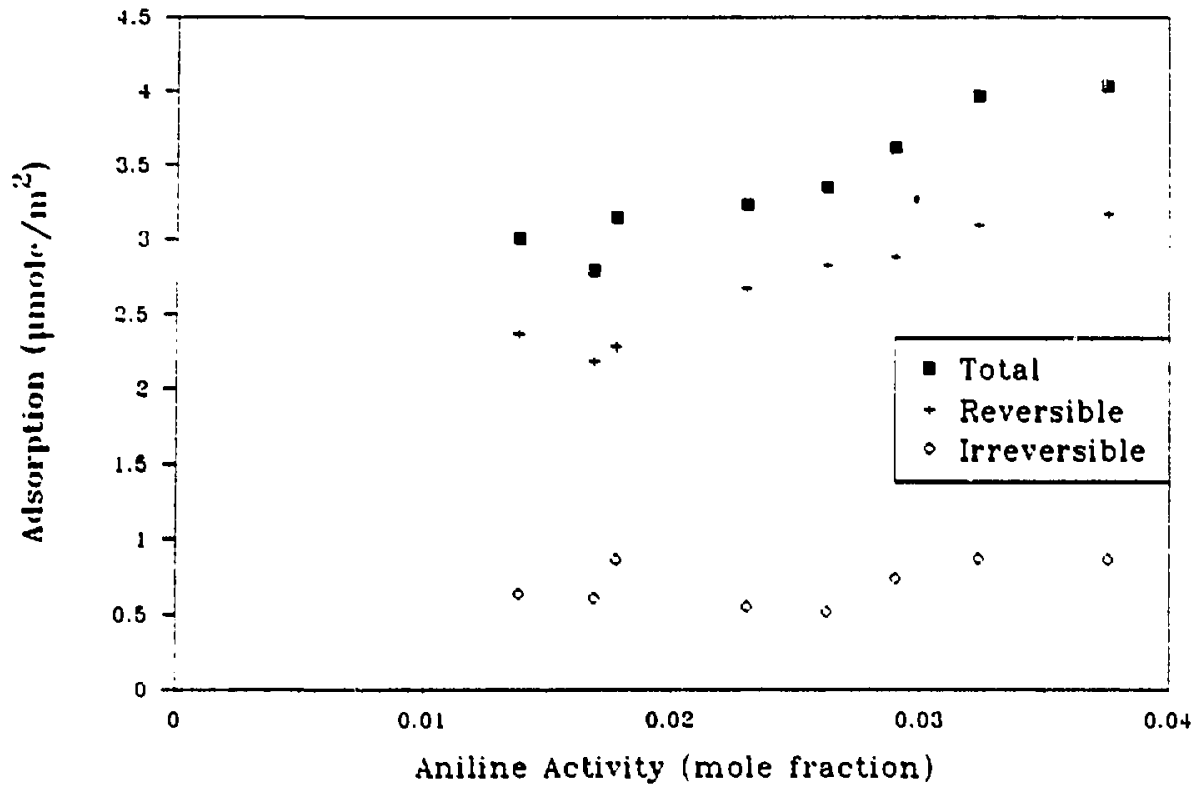

Figure 4.13 Total, Reversible and Irreversible Adsorption of Aniline from Water onto Columbia Carbon. Adsorption Contacts were for S Days at $80^{\circ} \mathrm{C}$. 
collected cannot be used to establish fundamental thermodynamic or kinetic parameters. The data can be used to estabiish a relative ordering of reactivities though, and this ordering may help indicate physiochemical properties that influence reactivities.

Two methods of comparing amounts of adsorption were used to establish an ordering. The first was to compare amounts of irreversible adsorption at one or more fixed values of activity, and the second was to compare amounts of irreversible adsorption at a fixed amount of total adsorption. The relative merits and liabilitiles of these methods are discussed below.

A qualitative ordering of reactivities was established by comparing amounts of irreversible adsorption over common ranges of activities. That is, if over a range of activities there is more irreversible adsorption of compound $A$ than compound $B$, then compound $A$ is designated as more reactive. This criterion leads to the ordering: p-methoxyphenol > 2,4-dimethylphenol " p-chlorophenol > phenol > aniline > p-nitrophenol " p-hydroxybenzaldehyde > nitrobenzene $=$ anisole.

The ordering can be made semiquantitative by comparing the amounts of irreversible adsorption at a single value of the solute activity. This method has the virtue that differences in amounts of adsorption of solutes at the same activity can be attributed to differences in surface 
interactions alone (see Appendix 2). A problem is that there are many types of surface interactions and, for each solute, the relative importance of various interactions may vary with activity. This means that the choice of an activity value affects the resulting scale. Another problem is that the activity coefficients may not be known with adequate precision (see Appendix 2).

Table 4.19 lists amounts of irreversible adsorption for various compounds at an activity of 0.002 . The choice of this value is arbitrary except for the fact that data are available near that activity for most of the adsorbates. These data were interpolated and/or smoothed to determine the numbers in the table.

A second method of ox....ung reactivities is to compare amounts of irreversible adsorption at one or more fixed values of the total adsorption. This approach has the virtue that the same number of solute molecules are on the carbon surfaces when the amounts of irreversble adsorption are compared 12 . Further, inaccuracies in the estimation of activity coefficients do not affect the results. A drawback is that there are different solute activities and hence, different driving forces for physisorption at the points of comparison.

12 Presumably the extents of the reactions causing irreversible adsorption depend on the surface concentrations of sorbate. 
Table 4.19 Properties of Substituted Benzenes

\begin{tabular}{lcccc} 
Compound & $\begin{array}{c}\text { Reactivity } \\
I^{*}\end{array}$ & $\begin{array}{c}\text { Reactivity } \\
I^{* *}\end{array}$ & $\begin{array}{c}\text { Boiling point } \\
(\circ \mathrm{C})\end{array}$ \\
\hline p-Methoxyphenol & 2.98 & 2.66 & 243 & 10.20 \\
2,4-Dimethylphenol & 2.19 & 2.03 & $210-212$ & 10.58 \\
p-Chlorophenol & 2.36 & 1.97 & 220 & 9.43 \\
Phenol & 1.70 & $1.74-2.03$ & 181.8 & 9.92 \\
Aniline & $\mathrm{NA}$ & 0.64 & 184.4 & $\mathrm{NA}$ \\
p-Hydroxybenzaldehyde & 0.20 & 0.25 & Sublimes & 7.62 \\
p-Nitrophenol & 0.20 & 0.25 & 279 & 7.15 \\
Anisole & 0 & 0 & 153.8 & NA \\
Nitrobenzene & 0 & 0 & 210.8 & NA \\
\hline
\end{tabular}

Estimated amount of irreversible adsorption at solute activity $=0.002$ (mole (raction).

- Estimated amount of irreversible adsorption at total adsorption $=3.0 \mu \mathrm{mole} / \mathrm{m}^{2}$ ".Values from Dean (1985). 
Table 4.19 also lists estimated amounts of irreversible adsorption for sorbates at a tutal uptake of 3.0 micromole $/ \mathrm{m}^{2}$. This uptake was chosen because data were available near that value. The surface coverage is on the order of 75\%, based on the plateau values for (revarsible) adsorption of nitrobenzene and methoxybenzene. The values for irreversible adsorption follow the qualitative ordering established earlier.

Included in Table 4.19 are the boiling points and acid ionization constants of the sorbates. As expected, boiling points do not correlate with reactivity. There is a rough correlation between $\mathrm{pK}_{\mathrm{a}}$ values and reactivities however. A tentative explanation for this trend is given in section 4.4 and in Chapter 5.

\subsection{Influences of Activity and surface coverage on Irreversible adsorption}

\subsubsection{Variations with solute Activity}

The data presented in section 4.2 showed that at less-than-monolayer coverages, irreversible adsorption of phenolics increases (or at least stays level) with increasing solute activity. The results of two experiments in which irreversible adsorption decreased with increasing solute activity are discussed in this section. 
Figure 4.14 shows total, reversible, and irreversible uptakes of phenol onto columbia carbon. The adsorption and desorption conditions were the same as those used to establish Figure 4.7 except for the initial phenol concentrations $\left(6.38 \times 10^{-3}, 6.38 \times 10^{-2}, 1.06 \times 10^{-1}, 3.19\right.$ $\left(610^{-1}\right.$, and $\left.6.38 \times 10^{-1} \mathrm{M}\right)$ and carbon weights $(0.027$ to $0.326 \mathrm{~g})$. The highast solute mole fraction at the end of a trial was 0.0097 , and an infinite dilution activity coefficient was used to estimate activity values.

A striking feature of Figure 4.24 is the internal maximum in the curve for irreversible adsorption. At solute activities below that of the maximum, irreversible adsorption increases with increasing reversible and total adsorption. This fact is consistent with a dependence of the extent of reaction 13 on surface concentrations. At solute activities above that of the maximum, there is a relatively slow decline in irreversible adsorption to a value near zero. The phenomena impeding irreversible adsorption are not apparent, but it is possible to rationalize the trend.

As mentioned in Section 4.2 , several investigators have postulated that as activity increases, physisorbed molecules reorient themselves on a carbon surface. The presumed orientations are flat on the surface and well as thermodynamic factors in these experiments. 


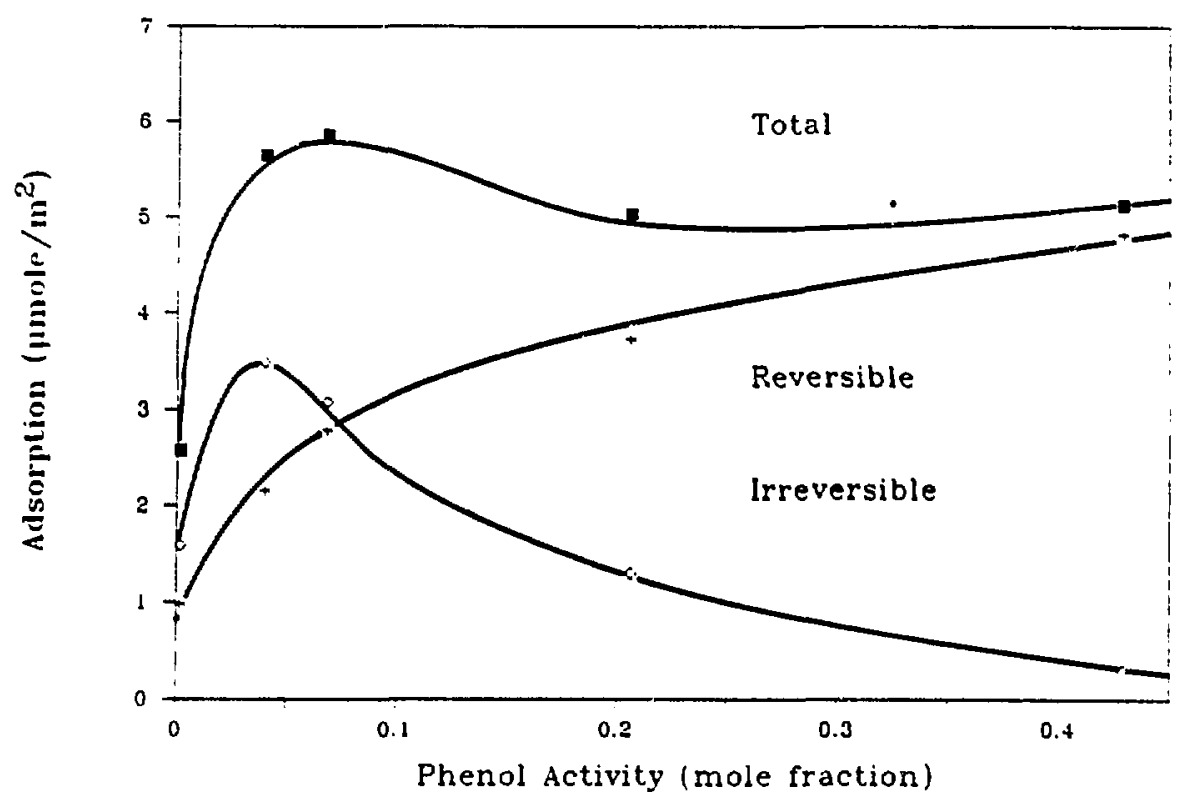

Figure 4.14 Total, Reversible and Irreversible Adsorption of Phenol from Water onto Columbia Carbon. Adsorption Contacts were for 5 Days at $80^{\circ} \mathrm{C}$. 
perpendicular to the surface at lower and higher activities, respectively. It is possible that phenol is reactive in only the former orientation. This would be consistent with the conclusion reached in section 4.1 .1 that activated carbon participates in the reactions causing irreversibilities.

If the suppositions stated in the last paragraph are true, the dynamics and equilibria of physisorption should greatly influence irreversible uptakes. Early in an adsorption trial and at any time when the solute activity in a carbon pore was low, irreversible reactions would proceed. At sufficiently high solute activities and sufficiently long equilibration times, all physisorbed molecules would be perpendicular to the surface and nonreactive. Thus, mass transfer resistances as well as equilibrium driving forces would influence irreversible uptakes, and an internal maximum in the irreversible adsorption curve would be possible.

The curve for reversible adsorption increases monotonically in Figure 4.14. There is no inflection or discontinuity apparent to indicate reorientation of sorbate molecules, but this may be due to the paucity of data. At the highest activity represented, each reversibly sorbed molecule occupies $0.34 \mathrm{~nm}^{2}$ on the average, which is less than the area expected for a flat orientation ( $\geq 0.40 \mathrm{~nm}^{2}$ ). 
Thus, the orientations of reversibly adsorbed molecules are probably different at relatively high and low solute activities.

Combination of the curves for irreversible and reversible adsorption yields the total adsorption curve. At the internal maximum and on the average, a sorbate molecule occupies $0.28 \mathrm{~mm}^{2}$ of the original surface. The molecules cannot all be lying tlat in a single layer. The molecules could be adsorbed in multiple layers, in a perpendicular orientation, and/or in a condensed layer of coupled molecules. In any event, irreversible adsorption can lead to substantial increases in total uptakes.

Figure 4.15 shows adsorption curves for hydroguinone at $25^{\circ} \mathrm{C}$. Hydroquinone oxidizes readily in neutral pH aqueous solution, and precautions were taken to minimize the amount of oxidation. Hydroguinone solutions of $5.73 \times 10^{-3}, 2.86$ $\times 10^{-2}, 5.73 \times 10^{-2}, 2.86 \times 10^{-1}$, and $5.73 \times 10^{-1} \mathrm{M}$ were prepared with water that had been sparged with nitrogen, and trials were initiated under a nitrogen atmosphere. Dark brown vials were used to shield the mixtures from light. Carbon weights varied between 0.027 and $0.50 \mathrm{~g}$.

Even at $25^{\circ} \mathrm{C}$, irreversible adsorption was facile at low activities. Comparison of Figures 4.15 and 4.7 shows that at activities less than 0.005 , irreversible uptakes of 


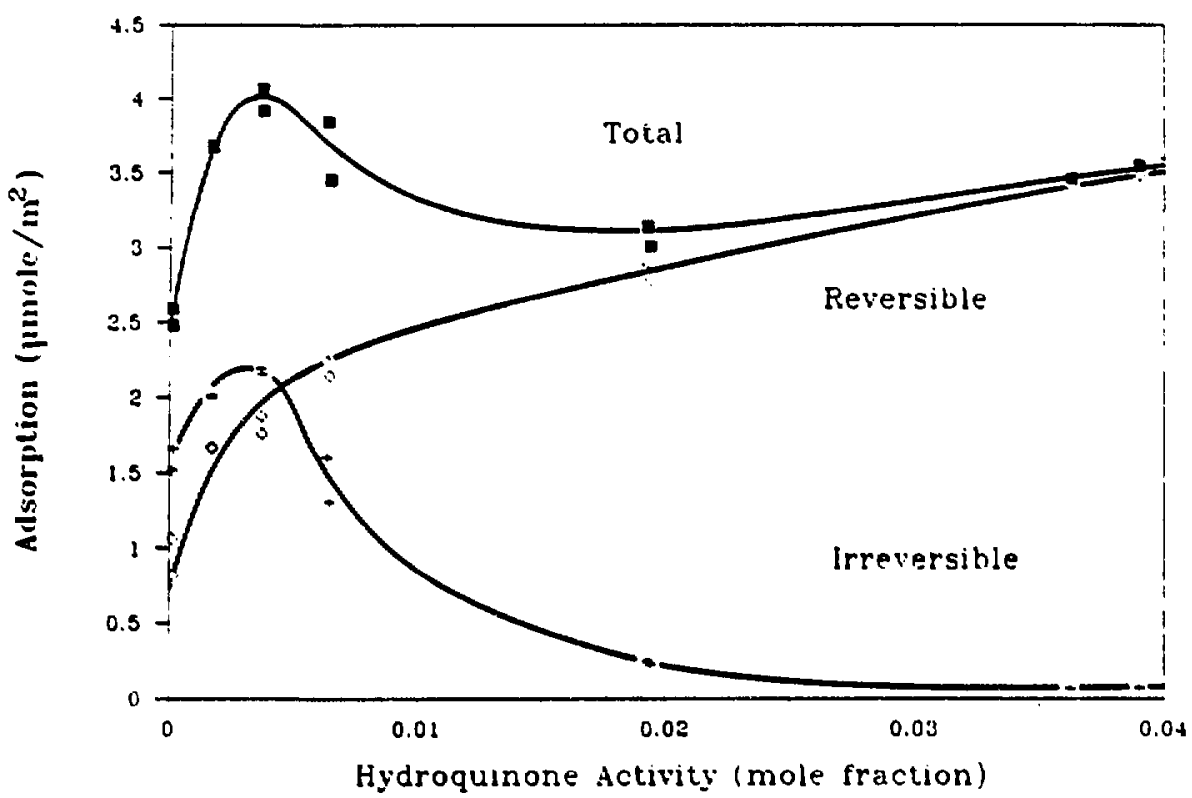

Figure 4.15 Total, Reversible and Irreversible Adsorption of Hydroquinone from Water onto Columbia Carbon. Adsorption Contacts were for 10 Days at $25^{\circ} \mathrm{C}$. 
phenol at $80^{\circ} \mathrm{C}$ and of hydroquinone at $25^{\circ} \mathrm{C}$ are comparable. Since the reaction rates should increase with temperature, hydroquinone is probably much more reactive than pherol.

The shapes of the corresponding curves in Figures 4.14 and 4.15 are very similar. Further, the maximum irreversible uptakes occur at comparable values of reversible adsorption. These facts are consistent with the postulated role of physisorption equilibria in determining irreversible uptakes.

\subsubsection{Repetitive Loading and Regeneration}

Repetitive cycles of phenol adsorption and desorption were carried out with $20 \times 40$ mesh Filtrasorb-100 carbon that had been exposed to the atmosphere for approximately 10 weeks. In each cycle, between 1.8 and 2.0 liter of 6.38 $x 10^{-3} \mathrm{M}$ solution of phenol in water was contacted with a sample of the carbon in a 2 liter erlenmeyer flask. The vessel was maintained at $80^{\circ} \mathrm{C}$ without agitation. After 5 days the mixture was vacuum filtered, and the carbon was contacted with acetone in batch desorption steps. The carbon was then washed with water in several batch contacts until no acetone could be detected after at least 10 hours of contact. The carbon was vacuum filtered and a small portion was removed immediately prior to the next adsorption contact. 
Table 4.20 shows total, reversible, and irreversible uptakes, and the final concentration of phenol in the aqueous phase for each of 3 cycles. The adsorption amounts are reported on the basis of the initial dry carbon weight ( $4.0 \mathrm{~g})$, less the dry weight of carbon removed during previous cycles. Sorbate that was not removed during regeneration would add to the carbon weight, but was not accounted for.

The total and irreversible uptakes reported for the first cycle are lower than those reported for F-100 in Table $4.3^{14}$. The same initial solution concentration and the same ratio of liquid to solid ( $\mathrm{ml} / \mathrm{gdc}$ ) were used in both tests. Presumably, F-100 was oxidized during exposure to atmospheric oxygen and was rendered less reactive in the repetitive regeneration experiment. Mattson and Mark (1971) discuss studies on "atmospheric aging" of carbons and conclude that acidic surface oxides form. Filtrasorb-100 was not recharacterized after the 10 week exposure, so it is not known if the oxygen or active hydrogen contents increased with aging.

The irreversible uptakes reported in Table 4.20 decreased with successive cycles, but there was still a significant amount in the third cycle. These facts indicate that the surface reactivity is only mildly

14 A surface area of $800 \mathrm{~m}^{2} / g d c$ was used to calculate the values in Table 4.3 
Table 4.20 Repetitive Adsorption and Regeneration Trials*

\begin{tabular}{ccccc} 
& \multicolumn{3}{c}{ Adsorption (mmole/gdc) } & \\
\cline { 2 - 4 } $\begin{array}{c}\text { Cycle } \\
\text { Number }\end{array}$ & Total & Reversible & Irreversible & $\begin{array}{c}\text { Final Phenol Concentration } \\
\text { (mmole/1) }\end{array}$ \\
\hline 1 & 1.63 & 0.80 & 0.83 & 3.13 \\
2 & 1.41 & 0.89 & 0.52 & 3.72 \\
3 & 1.36 & 0.89 & 0.47 & 3.68 \\
\hline
\end{tabular}

Adsorption of phenol from water onto Filtrasorb-100 carbon. Adsorption contacts were for 5 days at $80^{\circ} \mathrm{C}$, and the carbon was regenerated with acetone.

"Adsorption amounts are based on the dry carbon weights $4.00,3.79$, and $3.57 \mathrm{~B}$ for cycles 1,2 , and 3 respectively.

"Two liters of $6.38 \mathrm{mmole} / \mathrm{L}$ solution was used to initiate each of the first two adsorption contacts and $1.8 \mathrm{~L}$ of that solution was used to initiate the third. The concentrations reported in the table are those at the ends of the adsorption contacts. 
affected by irreversibly adsorbed compounds. As will be discussed in Sections 4.5 and 5.1 , it is possible that some of the sorbate included in the irreversible uptakes was removed by acetone. Further, the cumulative irreversible uptake ( $1.82 \mathrm{mmole} / \mathrm{gdc}$ ) is less than that needed to form a monolayer (2.95 mole/gdc, assuming an average surface coverage of $0.45 \mathrm{~nm}^{2}$ per molecule), so at least part of the original surface was available for reaction in the second and third cycles.

Total uptakes decreased with successive cycles, if only by small fractions of the cumulative amounts of irreversible adsorption. The decreases in capacity are consistent with observations made in other studies involving the adsorption of phenol from water. Sutikno and Himmelstein (1983), Chang and Savage (1981), Goto et al. (1986), and Cooney et al. (1983) all found that the capacities of carbon beds decreased during several cycles of adsorption and solvent regeneration. In general, the rate of change of capacity with cycle number decreased with increasing cycle number. The data of sutikno and Himmelstein show continual decreases in capacity over more than 45 cycles, although the changes are very gradual for large cycle numbers. Magne and walker (1986) found decreases in capacity with cycle number for batch adsorptions followed by solvent and/or thermal regenerations. They showed that $\mathrm{N}_{2}$ and $\mathrm{CO}_{2}$ BET surface 
areas were lowered by amounts that implied that decomposition products of irreversibly adsorbed phenol blocked pore apertures. The gradual changes in capacity observed in these studies are consistent with changes to the adsorbent due to slow sorbate reactions at the adsorption temperatures used $\left(12-30^{\circ} \mathrm{C}\right)$.

\subsection{Influences of Liquid-phase properties on Irreveraible Adsorption}

The nature of the liqgid or solvent phase can greatly influence uptakes of phenolic compounds by activated carbons. Temperature and activity effects have already been discussed. This section explores the influences of a nonaqueous solvent, of $\mathrm{pH}$, and of molecular oxygen on uptakes of phenol by columbia carbon.

\subsubsection{Adsorption from Heptene}

Adsorption of phenol from heptane by columbia carbon under a variety of conditions was studied. Total, reversible, and irreversible uptakes were determined for contacts lasting 5 and 14 days at both 25 and $80^{\circ} \mathrm{C}$. For each contact time and temperature, trials were initiated with solutions containing $3.83 \times 10-3,1.91 \times 10-2,3.83 \times$ $10-2,7.66 \times 10-2$, and $1.91 \times 10-1 \mathrm{M}$ phenol. All carbon samples weighed close to $0.3 \mathrm{~g}$, and the experimental procedures discussed in section 4.1.4.1 were followed. 
Figures $4.16 a b c$ show total, reversible, and irreversible uptakes, respectively, for the four sets of experimental conditions. At a given phenol activity, the amounts of total and irreversible adsorption increase with time and/or with temperature, while the amounts of reversible adsorption are not highly dependent on those variables 15 . These facts reinforce the major conclusions reached in Chapter 2 regarding the cause of irreversibilities in the adsorption of phenolics from water. In particular, the results for adsorption from heptane show that increases in carbon loadings at long times and at higher temperatures are due primarily to increases in irreversible adsorption and not to biological contamination or slow increases in reversible uptakes. Hence, surface reactions are implicated.

Comparison of uptakes of phenol onto columbia from heptane with those from water are revealing. Figures $4.17 a b c$ compare data from Figures $4.16 a b c$ and Figure 4.7 . Total uptakes are shown in Figure $4.17 \mathrm{a}$ and are best understood as composites of reversible and irreversible uptakes.

15 There is an anomaly in Figure 4.20b. Reversible uptakes probably should decrease with increasing irreversible uptakes (see section 4.4.3), but the reversible uptakes at $80^{\circ} \mathrm{C}$ are slightly higher than those at $25^{\circ} \mathrm{C}$. This effect is small and is not critical to the statements made above. 


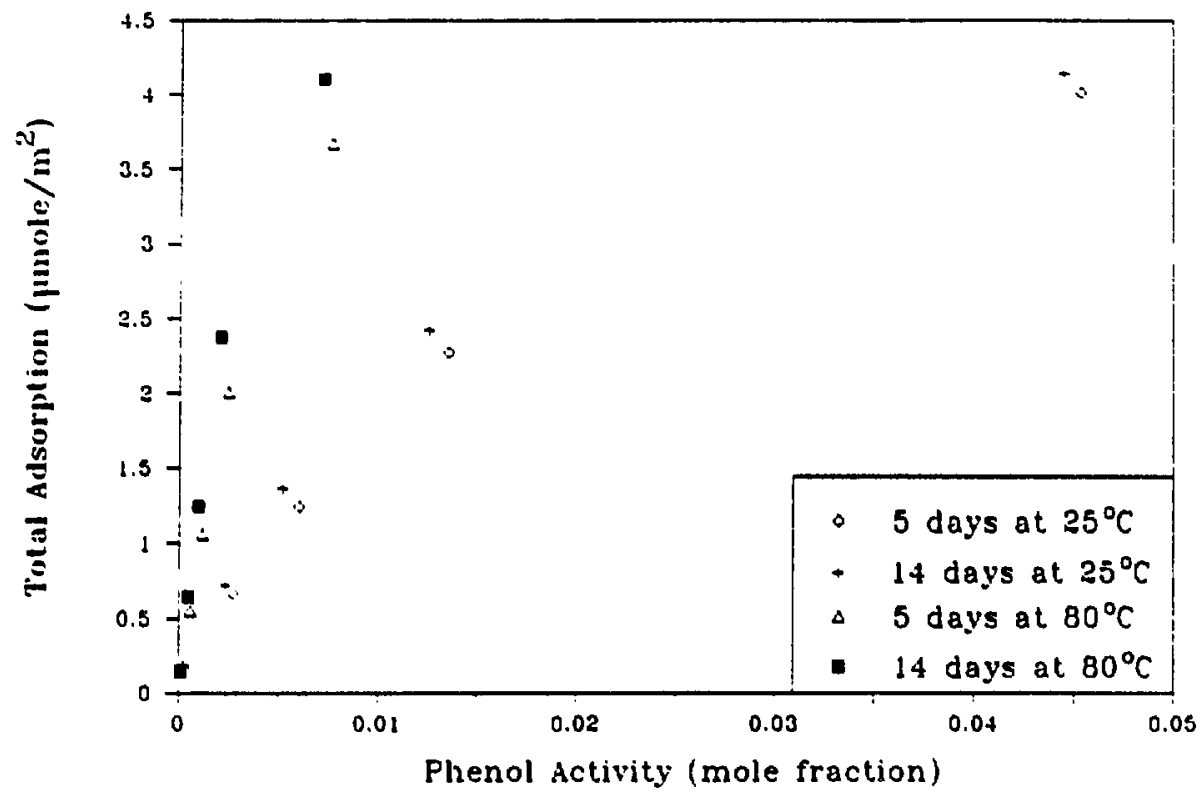

Figure 4.16a Total Uptakes of Phenol from Heptane onto Columbia Carbon. Adsorption Contact Times and Temperatures are Listed in the Legend. 


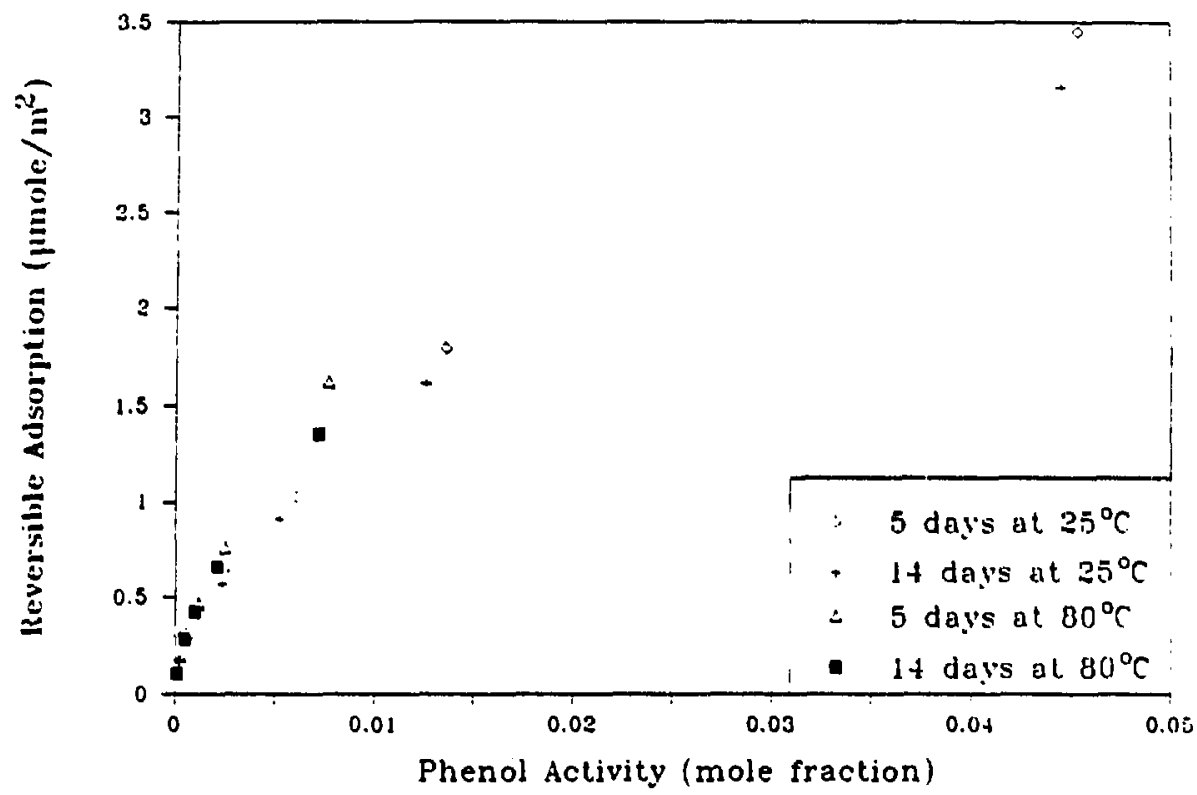

Figure 4.16b Reversible Uptakes of Phenol from Heptane onto Columbia Carbon. Adsorption Contact Times and Temperatures are Listed in the Legend. 


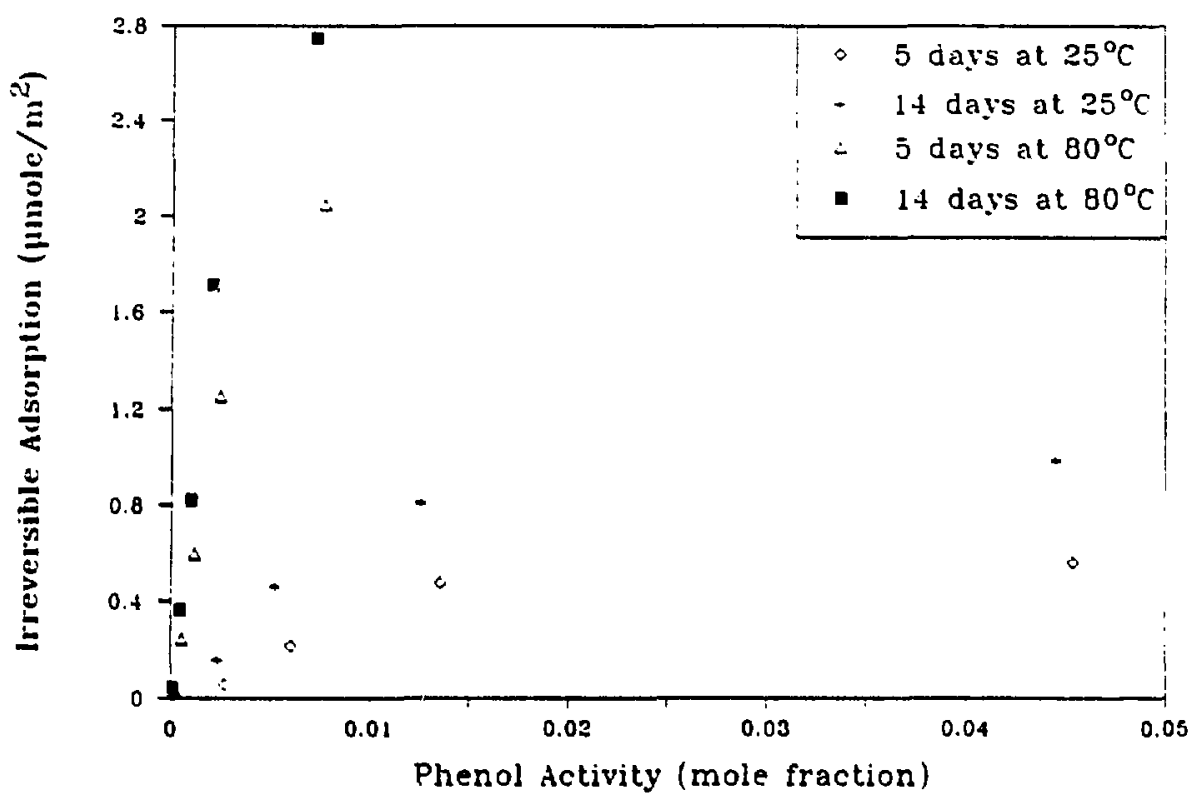

Figure 4.16c Irreversible Uptakes of Phenol from Heptane onto Columbia Carbon. Adsorption Contact Times and Temperature are Listed in the Legend. 


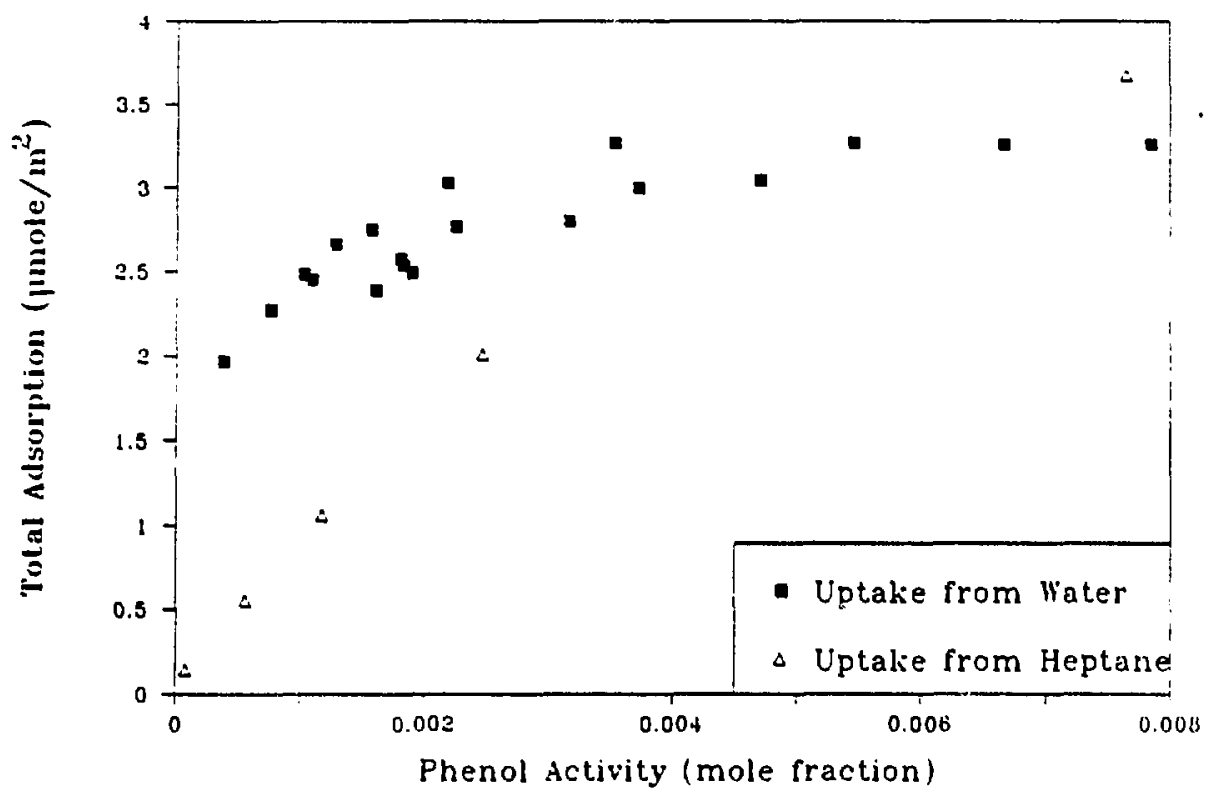

Figure 4.17a Total Uptakes of Phenol from Water and from Heptane onto Columbia Carbon. Adsorption Contacts were for 5 Days at $8^{\circ} \mathrm{C}$. 


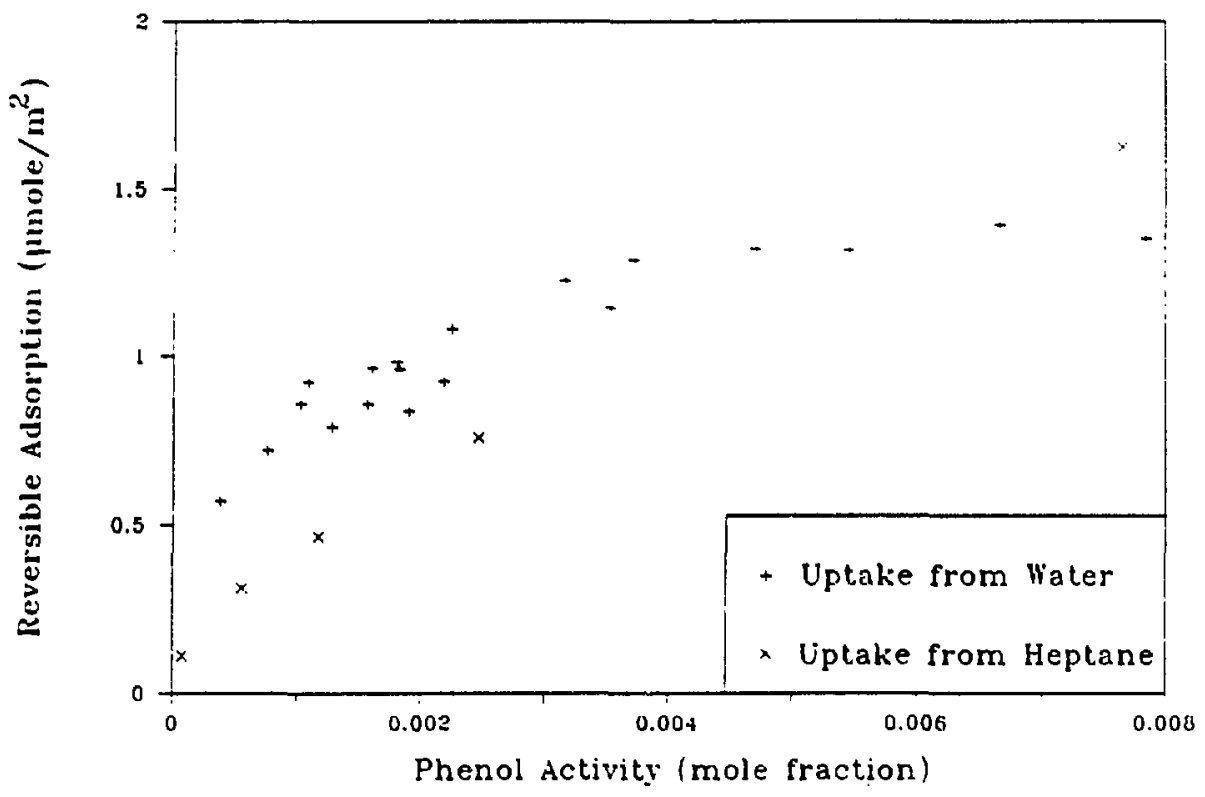

Figure 4.17b Reversible Uptakes of Phenol from Water and from Heptane onto Columbia Carbon. Adsorption Contacts rere for 5 Days at $80^{\circ} \mathrm{C}$. 


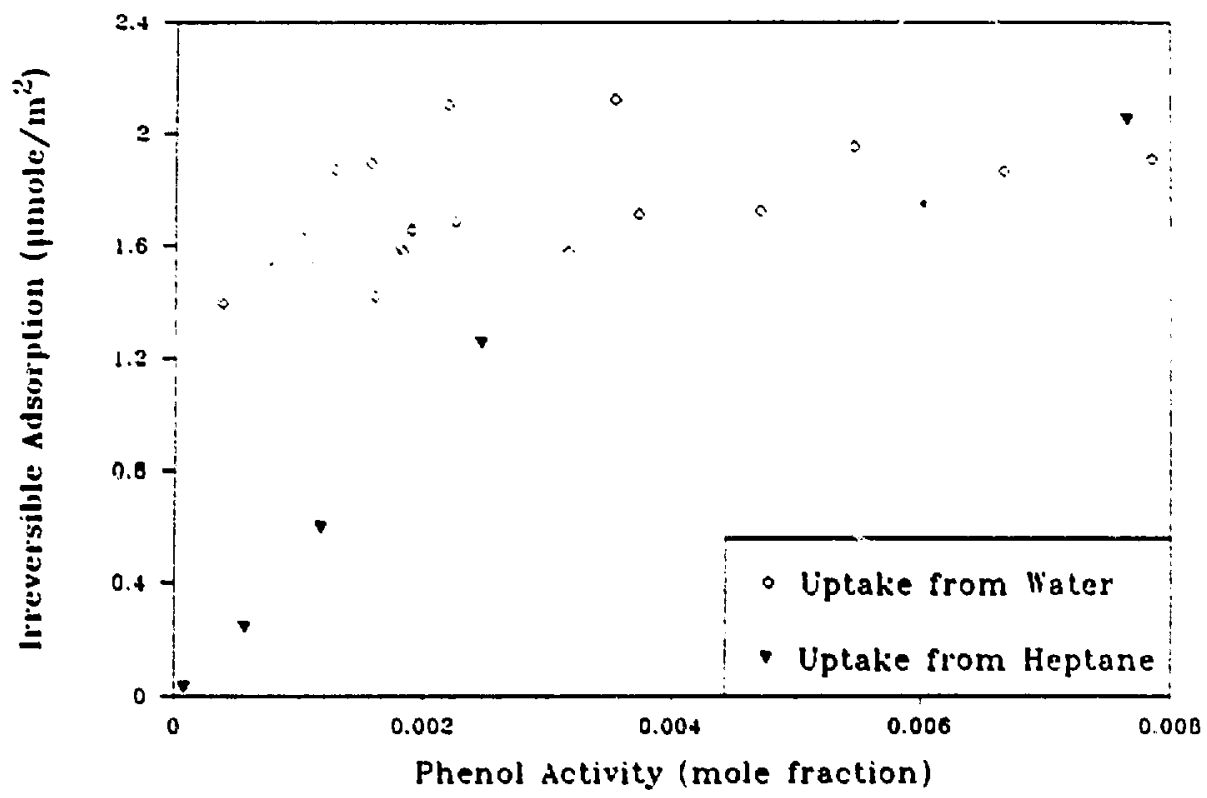

Figure 4.17c Irreversible Uptakes of Phenol from Water and from Heptane onto Columbia Carbon. Adsorption Contacts were for 5 Days at $80^{\circ} \mathrm{C}$. 
Figure $4.17 \mathrm{~b}$ shows that, at lower activities, there is less reversible adsorption of phenol from heptane than from water. Heptane competes more effectively than water for adsorption sites, which indicates that most of the adsorption of phenol is onto the nonpolar graphitic planes. The later conclusion is supported by the fact that the isotherms are concave to the abscissa (see Kipling, 1965, p 125). The rationale for the data point showing greater uptake from heptane than from water at higher activity is not apparent.

Figure 4.17c shows irreversible uptakes from the two solvents. The uptakes from heptane are lower than those from water, except at the highest activities. The adsorption amounts may reflect dependence of reaction rates on surface concentrations and/or influences of acid-base equilibria in water. These possibilities are discussed in sections 4.4 .2 and 5.2.3. In any event, irreversible uptakes are influenced by the solvent, and the reactions proceed during adsorption from organic as well as from aqueous media.

\subsubsection{EEfects of $\mathrm{pH}$}

Adsorption of phenol from three buffer solutions and from unbuffered water was monitored in order: to study the influence of solution $\mathrm{pH}$ on irreversible uptakes. The buffer solutions had $\mathrm{pH}$ values of $1.3,8.0$, and 12.0 and 
the compositions listed in Table 4.21. Phenol was added to each aqueous medium to yield $6.38 \times 10^{-3} \mathrm{M}$ solutions. Each solution was contacted with carbon samples weighing from 0.01 to $0.05 \mathrm{~g}$, in the manner described in section 4.1 .4 .1 . After the adsorption contacts, $\mathrm{pH}$ values of the unbuffered mixtures ranged from 7.0 to 7.9 . Regenerations were accomplished according to the procedures detailed in Section 4.1 .4 .1 .

Figures 4.18 abc compare total, reversible, and irreversible uptakes respectively for the four types of mixtures. Solubility data that would allow estimation of activity coefficients were not available, so the abscissa of each figure is phenol concentration. The uptakes from the unbuffered mixtures and those from the mixtures at pH 8 are very similar, which indicates that small variations in pH are inconsequential. Wider variations in pH markedly affect reversible and irreversible uptakes.

Figure $4.18 \mathrm{~b}$ shows that reversible uptakes are lower at $\mathrm{pH} 12.1$ than for $\mathrm{pH}$ values below the $\mathrm{pk}_{\mathrm{a}}$ of phenol $\left(\mathrm{pK}_{\mathrm{a}}=\right.$ 9.99, Dean, 1985). At $\mathrm{pH} 12.1$, phenol is almost completely dissociated. Phenolate anion has more affinity for the aqueous phase (i.e. a lower activity coefficient) than does phenol and would experience electrostatic repulsion from other anions in the surface layer. Reduced total uptakes onto carbon were observed for phenol and for $p$-nitrophenol 
Tabic 4.21 Aqueous Buffer Solutions Used in Adsorption Experiments

\begin{tabular}{cc} 
Composition & Messured pH Value \\
\hline $1.59 \times 10-1 \mathrm{M} \mathrm{KCl}$ & 1.8 \\
$4.13 \times 10^{-2} \mathrm{M} \mathrm{HCl}$ & \\
$1.30 \times 10-3 \mathrm{M}\left(\mathrm{NH}_{4}\right)_{2} \mathrm{CO}_{3}$ & 8.0 \\
$2.37 \times 10^{-2} \mathrm{M} \mathrm{NH} \mathrm{NCO}_{3}$ & \\
$1.61 \times 10-1 \mathrm{M} \mathrm{KCl}$ & 12.0 \\
$3.87 \times 10^{-2} \mathrm{M} \mathrm{NaOH}$ & \\
\hline
\end{tabular}




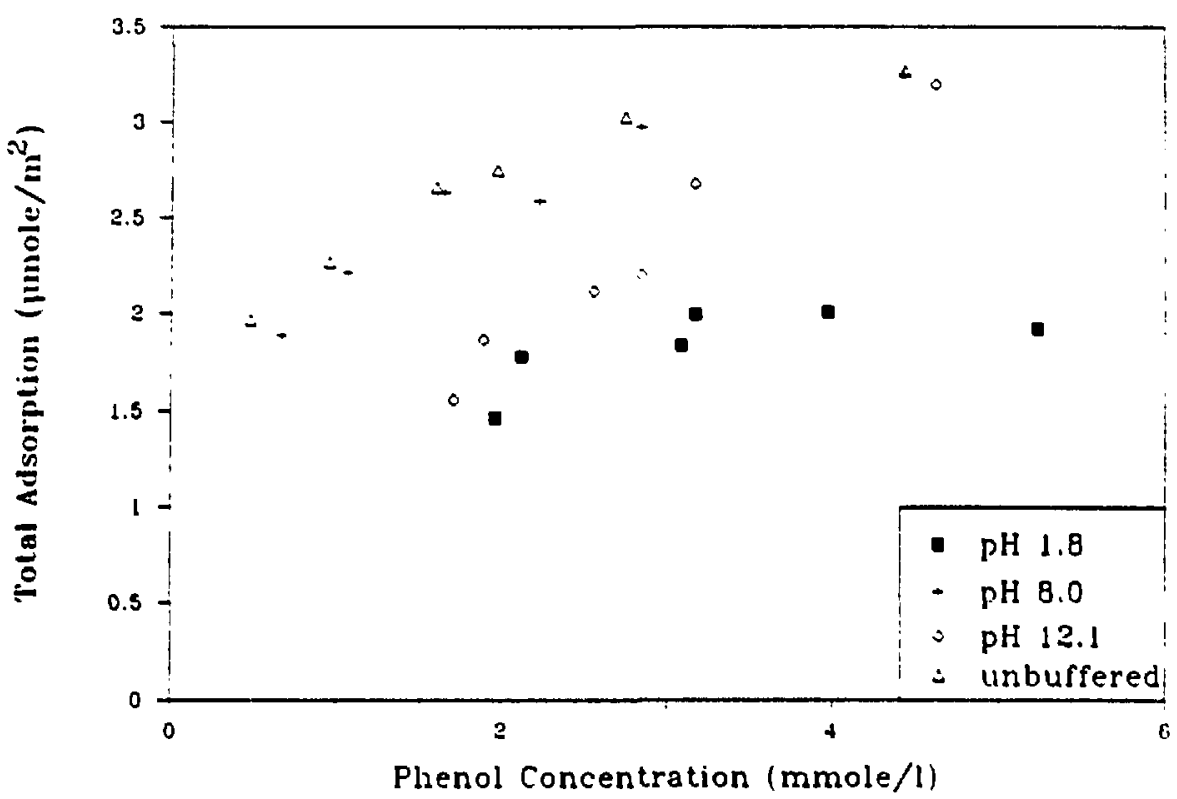

Figure 4.18a Total Uptakes of Phenol from Aqueous Solutions with Various Values of pH. Adsorption Contacts were for 5 Days at $80^{\circ} \mathrm{C}$. 


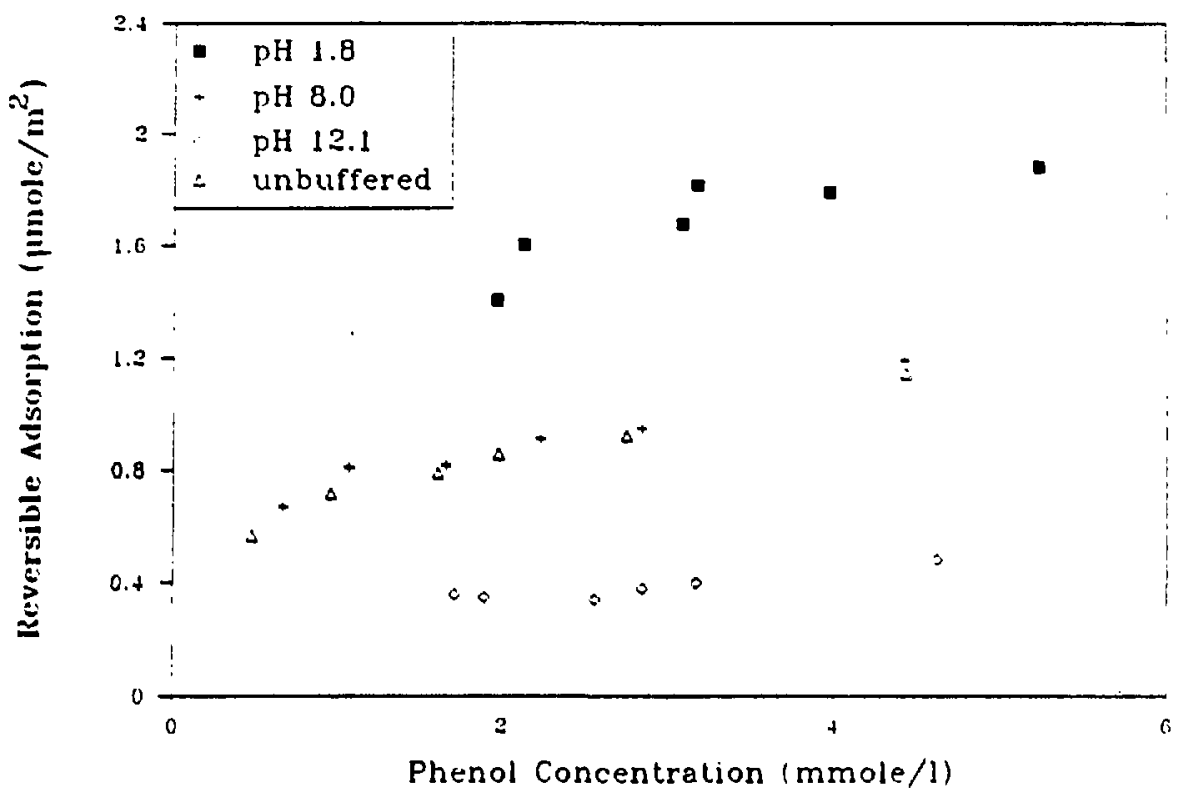

Figure 4.18b Reversible Uptakes of Phenol from Aqueous Solutions with Various Values of pH. Adsorption Contacts were for 5 Days at $80^{\circ} \mathrm{C}$. 


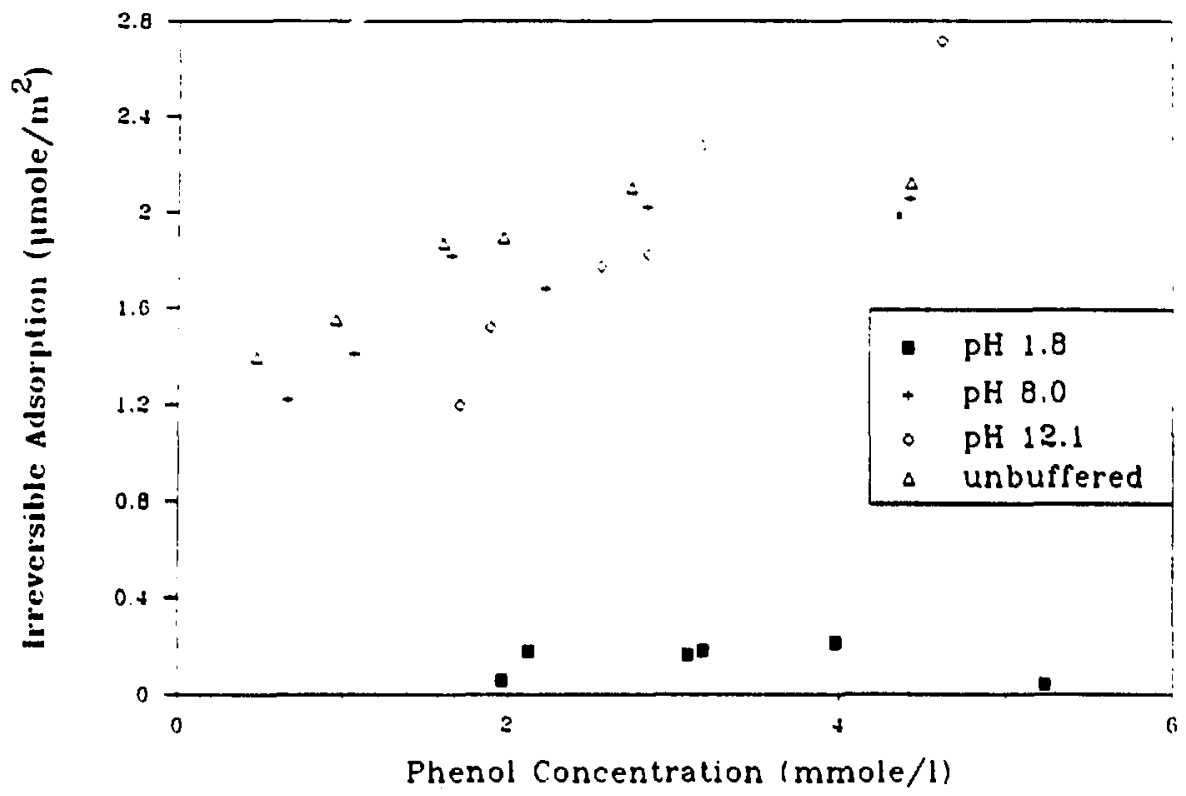

Figure 4.18c Irreversible Uptakes of Phenol from Aqueous Solutions of Various Values of pH. Adsorption Contacts were for 5 Days at $80 \cdot \mathrm{C}$. 
at $\mathrm{pH}$ values above their respective $\mathrm{pk}_{\mathrm{a}}$ values, by snoeyink et al. (1969) and by Müller et al. (1980), as well as in studies referenced by those investigators.

Reversible uptakes also increased with decreasing pH at values below the $\mathrm{pK}_{\mathrm{a}}$ of phonol. similar observations were made for total uptakes of p-nitrophenol onto carbon by Mattson et al. (1969) and were attributed to decreases in solubility with decreasing pH. Although solubility data are not available, it is possible that phenol activities were higher in the $\mathrm{pH} 1.8$ buffer, particularly because of the relatively high ionic strength. Changes in surface charge with pH can also affect reversible uptakes of phenolics, but the influences should be minimal at $\mathrm{pH}$ values well below the solute pka (Müller et al., 1980).

In contrast, Figure $4.18 \mathrm{C}$ shows that irreversible uptakes at pH 1.8 are much lower than those at higher pH values. Since reversible uptakes are less than or equal to that for the maximum in irreversible adsorption in Figure 4.14, activity effects and/or differences in surface orientation are probably not responsible. Rather, the data indicate a $\mathrm{pH}$ dependerice of the reactions leading to irreversible adsorption. Changes in the nature of the surface layer due to adsorption of ions and the form of the phenolic sorbate could affect these reactions.

Base-catalyzed reactions, acid-inhibited reactions, and 
reaction paths wherein phenolate ion participates in a rate-determining step are all possible reasons for lower irreversible uptakes at lower $\mathrm{pH}$.

Comparison of Figures $4.18 \mathrm{a}$ and $4.18 \mathrm{C}$ reveals that almost all of the adsorption at $\mathrm{pH} 12.1$ is irreversible. Since virtually all of the solute is phenolate ion and since there is much less reversible adsorption of phenolate ion than of molecular phenol, it seems reasonable to conclude that phenolate ion is more reactive than phenol.

The influence of $\mathrm{pH}$ on irreversible adsorption may help to explain some results already reported. oxidation of Columbia carbon by the means discussed in section 4.1.4.2 increased the surface acidity (as measured by active hydrogen), for example, and this factor may have been the primary reason for decreased irreversible uptakes. Further, the fact that at low activities there is more irreversible adscrption from unbuffered water than from heptane may result from the relative ease of forming anions in water. The results for adsorption fror heptane, however, indicate that formation of ions in bulk solution is not a prerequisite for irreversible adsorption.

\subsubsection{Availability of oxygen}

This section focuses on experimental observations about the influence of molecular oxygen on irreversible adsorption. Three types of solid-solution-vapor contacts 
were used to vary the amount of oxygen available during adsorption trials. The first type is that described in Section 4.1.4.1 and used to obtain most of the data reported in this chapter. In brief, phenol solutions were prepared with water equilibrated with the atmosphere, $15 \mathrm{ml}$ aliquots were added to $20 \mathrm{ml}$ scintillation vials (the actual total volume of each vial is $22.5 \mathrm{ml}$ ), and the adsorption contacts were initiated in the open atmosphere (so the headspace was filled with air when the vial was sealed). The amount of oxygen in a vial was reduced in the second type of contact. Phenol solutions were prepared with water that had been sparged for several minutes with nitrogen gas, $20 \mathrm{ml}$ aliquots were added to $20 \mathrm{ml}$ scintillation vials, and the adsorption contacts were initiated (and the vials sealed) under a nitrogen atmosphere. Oxygen was relatively plentiful in the vessels used for the third type of contact. Phenol solutions were prepared with water equilibrated with the atmosphere, $20 \mathrm{ml}$ aliquots were added to weighing vials of $95 \mathrm{ml}$ total volume, and the adsorption contacts were initiated in the open atmosphere. Assuming a dissolved oxygen content of $5.78 \times 10^{-3} \mathrm{ml} \mathrm{O} / \mathrm{ml} \mathrm{H}_{2} \mathrm{O}$ (solubility from Dear, 1985), there were respectively $0.068,0$, and 0.65 mmole of oxygen available in the solutions and headspaces of the three types of trials. All three types of contacts were at $80^{\circ} \mathrm{C}$ for 5 days. 
Carbon weights were adjusted so that adsorption contacts with the same ratio of liquid to solid (w/w), but different amounts of available oxygen could be compared. That is, for each trial run using the first type of adsorption conditions ( $15 \mathrm{ml}$ aliquots), a trial was run for each of the other types of contacts $(20 \mathrm{ml}$ aliquots) using $4 / 3$ as much carbon. All carbon samples were exposed to the atmosphere during the weighing process.

Liquid-solid phase separation and carbon regeneration were accomplished in the manner described in section 4.1.4.1. Aqueous phase-solid mixtures were transferred from the weighing vials to $20 \mathrm{ml}$ scintillation vials before centrifugation.

Total and irreversible uptakes were higher when more oxygen and more headspace were available, as is shoin in Figures $4.19 \mathrm{ac}$. This result is attributed to increases in the extents of chemical reactions anc not to increased losses of phenol to the vapor phase. Calculations showed that the amounts of phenol in the headspaces of contact vessels were negligible comparsd to uncertainties in the calculated uptakes. Further, the sampling procedures used in these trials were the same as those used for tests with XAD-4 in which tot? ? and reversible uptakes of phenol corresponded (see section 4.1.1). It seems likely, therefore, that molecular oxygen promotes irreversible adsorption. 


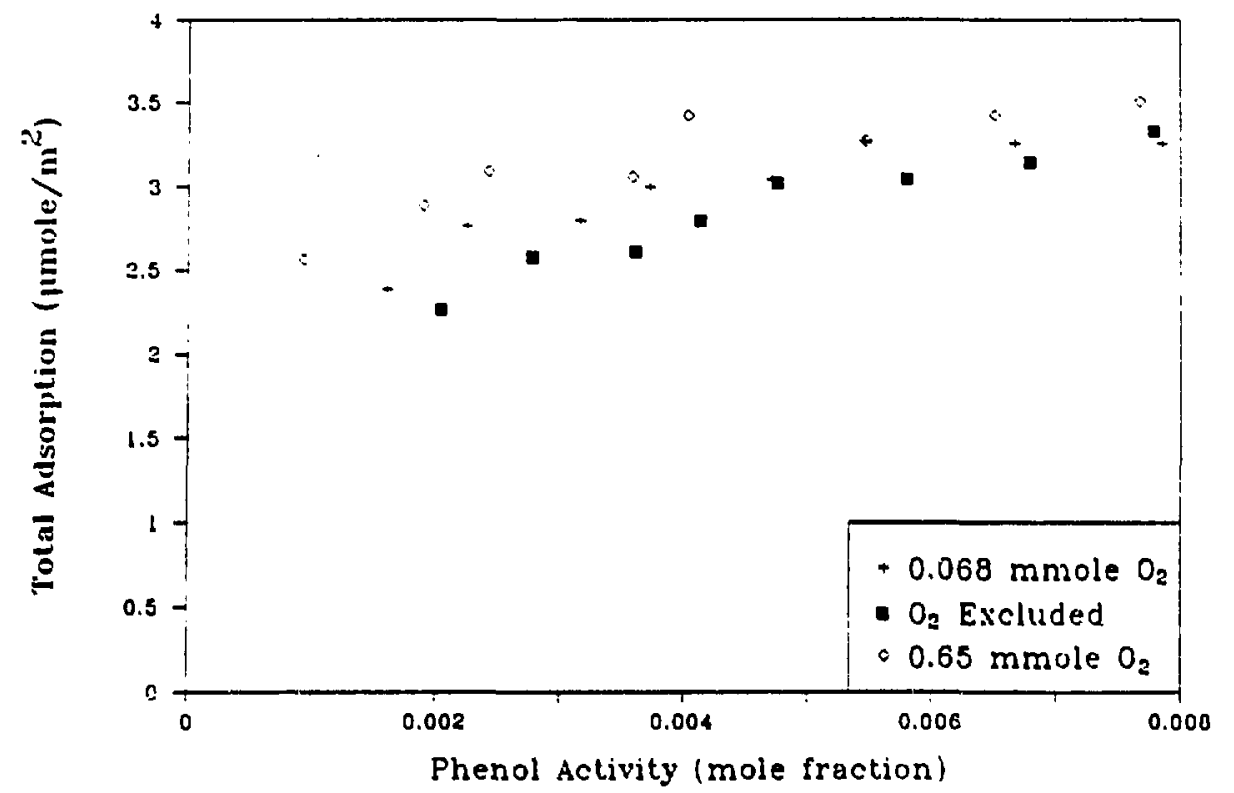

Figure 4.19a Total Uptakes of Phenol from Aqueous Solutions with Various Amounts of $\mathrm{O}_{2}$ Arailable in the Contact Vessels. 


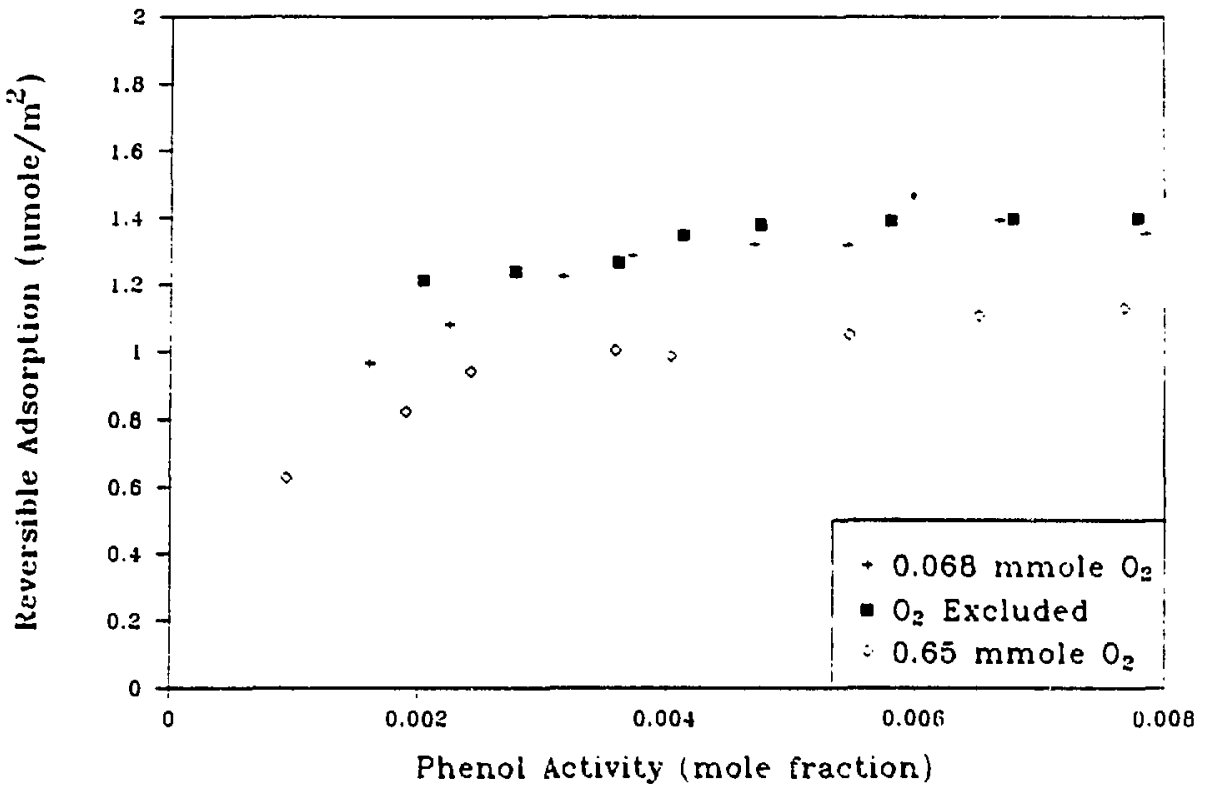

Figure 4.19t Reversible Uptakes of Phenol from Aqueous Solutions with Various Amounts of $\mathrm{O}_{2}$ Available in the Contact Vessels. 


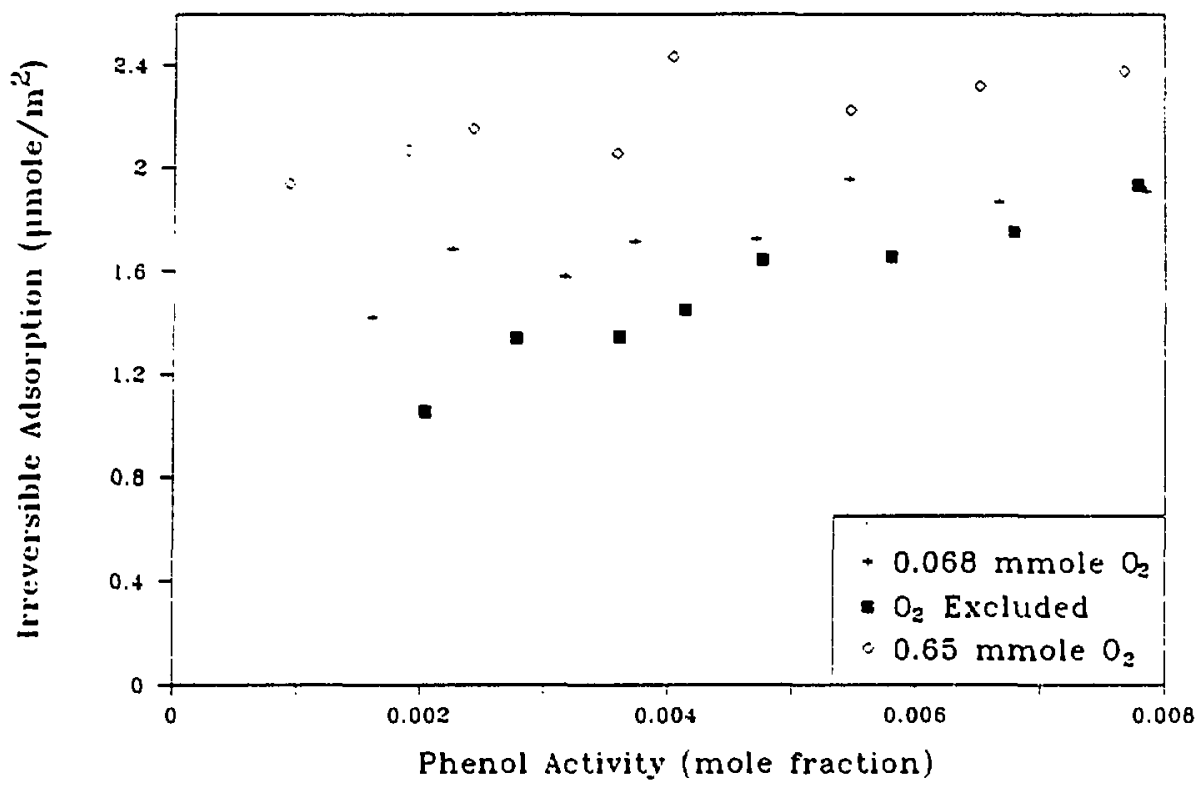

Figure 4.19c Irreversible Uptakes of Phenol from Aqueous Solutions with Various Amounts of $\mathrm{O}_{2}$ Available in the Contact Vessels. 
still, irreversible adsorption was prevalent in the trials in which oxygen was excluded from the liquid and vapor phases. This fact suggests that either molecular oxygen is not required in the surface reactions, or that oxygen was present during the trials. It is possible that oxygen was adsorbed onto the carbon samples before use and/or that oxygen was incompletely removed from water through sparging with nitrogen. Molecular oxygen may act as a catalyst, as discussed in chapter 5.

These results are consistent with the findings of seewald et al. (1983), which were discussed in chapter 1. In particular, seewald et al. reported greater amounts of chemisorption of phenol from air than from helium. The investigators did not provide evidence of irreversible adsorption from helium, but their statements indicate that there was some. More evidence is needed to determine whether irreversible adsorption of phenolics occurs under anerobic conditions.

Figure $4.19 b$ shows that reversible uptakes were generally lower when more oxygen was present. This may have been due to increased amounts of irreversibly adsorbed sorbate.

A similar set of trials was made, involving a wider range of solute activities. The same three types of adsorption contacts were used, except that $15 \mathrm{ml}$ rather than $20 \mathrm{ml}$ aliquots were added to the $95 \mathrm{ml}$ weighing vials. 
Therefore, there were 0.69 mole $\mathrm{O}_{2}$ available in the solution and the headspace of each trial using weighing vials. Initial phenol concentrations were $6.38 \times 10^{-3}$, $6.38 \times 10^{-2}, 1.06 \times 10^{-1}, 3.19 \times 10^{-1}$, and $6.38 \times 10^{-1} \mathrm{M}$, and, for the solutions initiated with $15 \mathrm{ml}$ aliquots, the carbon weights varied from 0.027 to $0.326 \mathrm{~g}$.

Figures 4.20abc show total, reversible, and irreversible uptakes respectively for the three types of adsorption contactsi6. The data for the first type of trial were presented in Figure 4.14 and discussed in Section 4.3. Again, total and reversible uptakes were generally higher, and reversible uptakes were lower when more oxygen was available. Thus, these data support the conclusions drawn in the discussion of Figures 4.19abc.

\subsection{Desorbable Reaction Producta}

As discussed in Chapter 1, Seewald et al. (1983) identified two desorbable reaction products in their thermal regeneration studies. In this work, several reaction products were identified in acetone that had been used to regenerate columbia carbon.

Phenol was adsorbed onto columbia from a solution of $0.191 \mathrm{M}$ phenol in heptane and then desorbed into acetone. A $0.3 \mathrm{~g}$ sample of Columbia was contacted with a $15 \mathrm{ml}$ aliquot of the phenol-heptane mixture and then with a total

16 The results of one trial in which $\mathrm{O}_{2}$ was excluded are not reported because the contact vessel leaked. 


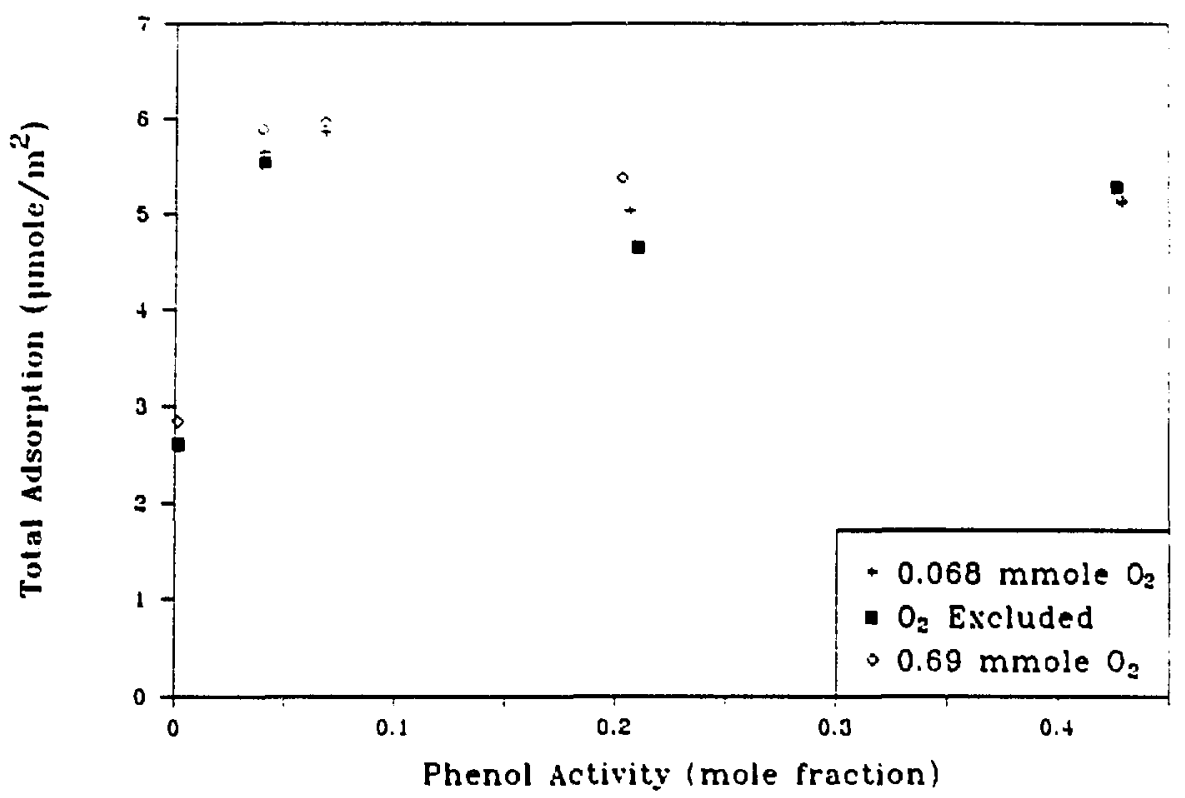

Figure 4.20a Total Uptakes of Phenol from Aqueous Solutions with Various Amounts of $\mathrm{O}_{2}$ Arailable in the Contact Vessels. 


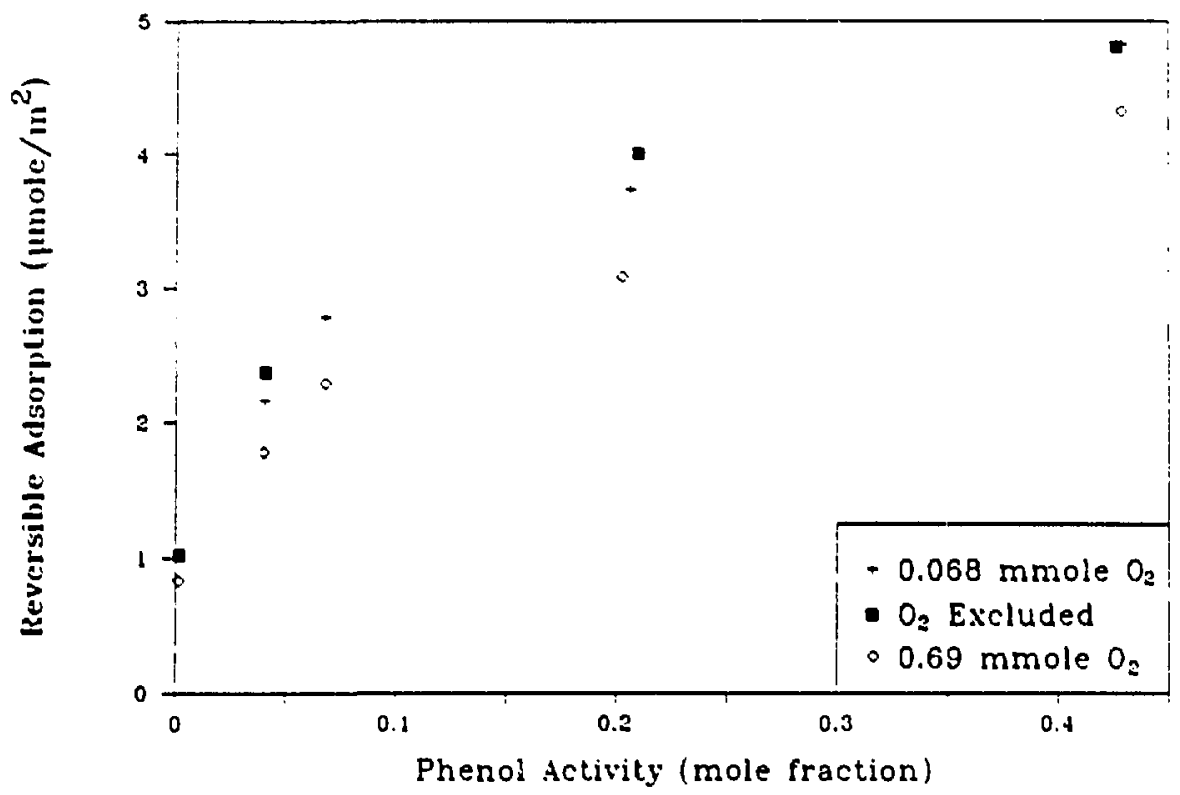

Figure 4.20b Reversible Uptakes of Phenol from Aqueous Solutions with Various Amounts of $\mathrm{O}_{2}$ Avallable in the Contact Vessels. 


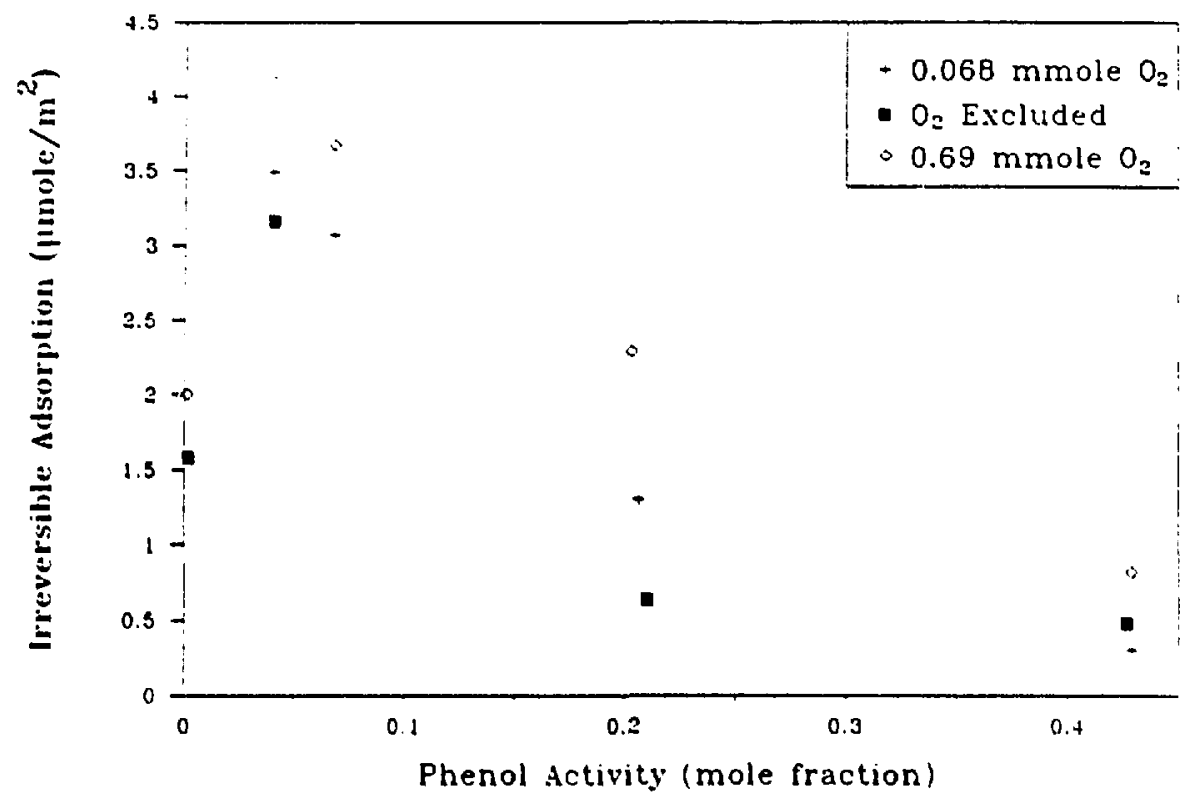

Figure 4.20c Irreversible Uptakes of Phenol from Aqueous Solutions with Various Amounts of $\mathrm{O}_{2}$ Available in the Contact Vessels. 
of $50 \mathrm{ml}$ of acetone, according to the procedures described in section 4.1.4.1. The total, reversible, and irreversible uptakes were $4.10,1.35$, and $1.75 \mathrm{micromoles}$ per square meter respectively. No polymers were detected in the heptane phase by means of GC (at $210^{\circ} \mathrm{C}$, with a porapak-Q column and flame ionization detection) or in the regenerant by means of HPLC (on the CN column17 with an aqueous mobile phase and detection of uv absorbance at 214 nm) 18 .

The regenerant mixture (concentration $7.31 \times 10^{-3} \mathrm{M}$ ) and a $7.45 \times 10^{-3} \mathrm{M}$ 'control' solution of phenol in acetone were concentrated prior to a mass-spectrometry probe analysis. Twenty milliliter aliquots of the solutions were placed in a vacuum oven at $30^{\circ} \mathrm{C}$ until the volumes were reduced to approximately $10 \mathrm{ml}$. Two milliliter aliquots of the resulting solutions were evaporated to dryness in a rotary vacuum concentrator (Savant Instruments Inc., model $\mathrm{RH}$ 20-12). A drop of acetone was added to each sample to facilitate transfer to the sample probe of a single-focusing, magnetic sector mass spectrometer (AEI Corp., Model MS 12).

A low-resolution, probe analysis of each sample was performed by Mr. Paul defur of the college of Chemistry Mass Spectrometry Laboratory at the University of

17 See Appendix 1.

18 See Appendix 1 for more details on the HPLC analyses. 
California, Berkeley. Each sample was volatilized slowly by raising the sample temperature from 25 to $300^{\circ} \mathrm{C}$ in the mass spectrometer. Eluted compounds were ionized by electron ionization.

Figures 4.21 ab show the resulting mass chromatograms for the regenerant and the control mixtures respectively. In each chromatogram. the abscissa is time (in hours:minutes) or correspondingly, the number of scans completed. The ordinate of a chromatogram is the relative intensity of the detector signal compared to the peak intensity in the top chromatogram in the same figure. To the right of each chromatogram is the range cf base mass to charge ratios examined. The last chromatogram in each figure is the reconstructed (or total) ion chromatogram.

The base mass to charge ratios for the chromatograms in Figure $4.21 \mathrm{a}$ indicate that phenol and polymers of composition $\mathrm{C}_{6 \pi} \mathrm{H}_{4 n+2} \mathrm{O}_{n}(n=2$ to 7$)$ were present in the regenerant mixture. The relative signal intensities show that the higher the molecular weight of a polymer, the less abundant it was. In contrast, Figure $4.21 \mathrm{~b}$ shows that phenol, but no polymers, were present in the control solution 19 . These facts indicate that polymers formed during the adsorption process and were recovered from columbia during regeneration.

19 The chromatograms corresponding to mass to charge ratios of 186 and 278 show only low levels of background noise. 
$\mathrm{n} / \mathrm{e}$ Fange

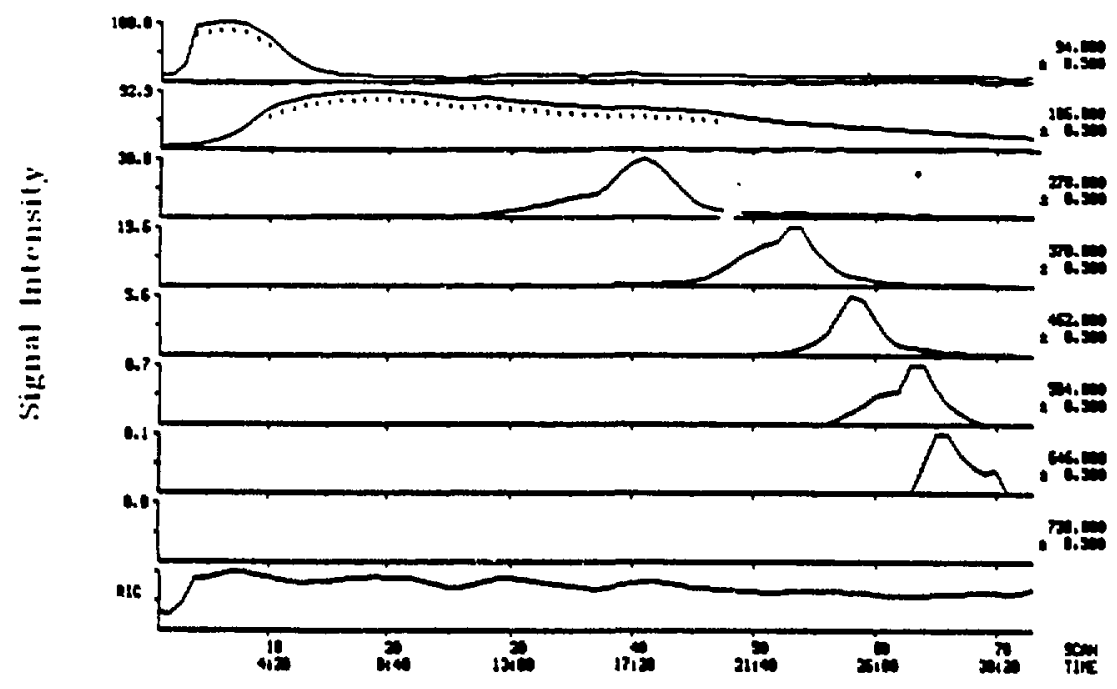

Figure 4.21a Mass Chromatograms Por a Regenerant Mixture. Phenol and Several Polymers ware Present. 
$\mathrm{m} / \mathrm{e}$ Range

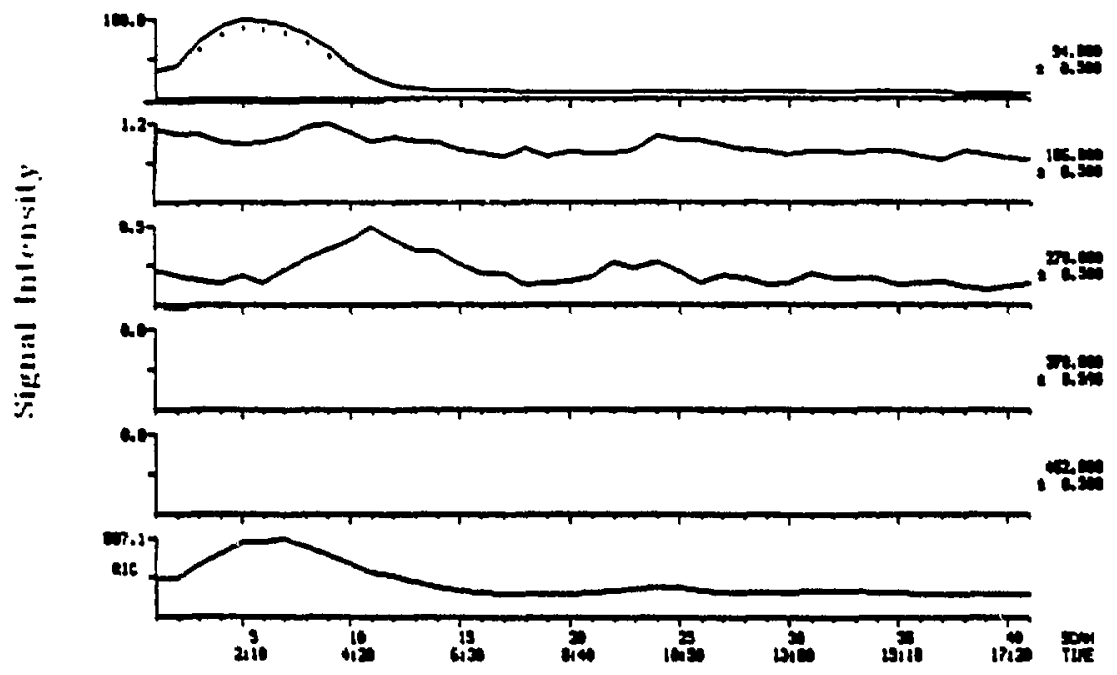

Figure 4.21b Mass Chromatograms for a Solution of Phenol in Acetone. No Polymers were Detected. 
The results of these experiments are consistent with the reaction process proposed in Chapter 5.

\subsection{References}

Anderson, R.E. and Hansen, R.D. Ind. Eng. Chem., 1955, 47, $71-75$.

Antonowicz, K. I. Shem. Phys,, 1962, 36, 2046-2053.

Antonowicz, K. Sarbon, 1964, 1, 111-115.

Banerjee, A.; Mazumdar, B.K. and Lahiri, A. Nature, 1962, $193,267-268$.

Brown, W.G., "Reductions by Lithium Aluminum Hydride", in organic Reactions, Volume VI, Adams, R., Ed., Wiley, New York, 1951.

Chanda, M.: O'Driscoll, K.F.; and Rempel, G.I. Reactive Polymers, 1985, 4, 39-48.

Chang, C.H., and Savage, D.W. Environ. Sci. Technol,, 1981, $15,201-206$

Chang, C.H.; Savage, D.W.; and Longo, J.M. J. Colloid and Interface science, 1981, 79, 178-191.

Chasanov, M.G.; Kunin, R.; and NcGarvey, F. Ind. Eng. Chem., 1556, 48, 305-309.

Chriswell, C.D.; Ericson, R.L.; Junk, A.G.; Lee, K.W.; Fritz, J.S. and Svec, H.J. Journal AWWA, 1977, 69, 669-674. 
Cookson, J.T, "Adsorption Mechanisms: The Chemistry of Organic Adsorption on Activated Carbon", in Carbon Adsorption Handbook, Cheremisinoff, P.N., and Ellerbusch, F., Eas., Ann Arbor Science Publishers, Ann Arbor, MI, 1978 .

Cooney, D.0.; Nagerl, A.; and Hines, A.L. Water Res., 1983, 12. 403-410.

Costa, C.A. and Rodrigues, A.E., "Adsorption of Ehenol on Adsorbent Resins and Activated Carbon: Equilibrium and Kinetic Studies in Bateh and Open Systems", in desorption at the Gas-solid and Liouid-solid Interface, Rouquerol, J. and Sing, K.s.พ., Eds., Elsevier Scientific Publishing Company, Amsterdam, 1982.

Cotton, F.A. and Wilkinson, G. Advanced Inorganic Chemistry a comorehensive Text, 4 th ed., John wiley \& Sons, NY, 1980. Coughlin, R.W., and Ezra, F.S. Environ. Sci. Technol., $1968,2,291-297$.

Coughlin, R.w., and Tan, R.N. Chem. Eng, Prog. Symp. Series, 1968, 64, No. 90, 207-214. Coughlin, R.W.; Ezra, F.S.; and Tan, R.N. I. colloid and Interface Science, 1968, 28, 386-396.

Crook, E.H.; MCDonnell, R.P.; and MCNulty, J.T. Ind. Eng. Chem.- Prod. Res. Dev., 1975, 14, 113-118. 
Dean, J.A. Ed. Lange's Handbook of chemistry, 13th ed., McGraw-Hill, NY, 1985.

Diamond Shamrock Corp., "Duolite S-761 Resinous Adsorbent", Redwood City CA, 1978.

Epstein, B.D.; Dalle-Molle, E.; and Mattson, J.B. Cacbon, $1971,2,609-615$.

Farrier, D.S.: Hines, A.I.: and WE, G, S.W. I. colleid and Intertace Seience, 1979, 62, 233-237.

Fox, C.R. Hydrocarben Proensing, 1978, 57, 11, 269-273. Garcia, A.A., Ph.D. Dissertation in Chemical Engineering, University of California, Berkeley, 1988. Garcia, A.A., Lniversity of California, Berkeley, Personal Communication, 1987.

Garten, V.A. and weiss, D.E. Bey, pure Appl, Cheme, 1957a, I. 69-122.

Garten, V.A. and Weiss, D.E. Austral, I chem,, 1957b, 10, 309-328.

Giles, C.H.; MacEwan, T.H.: Nakhwa, S.N.; and Smith, D. J. chem. Sec., 1960, 3973-3993.

Goto, M.; Hayashi, N.: and Goto, S. Environ. Sci. Technol.: 1986 , 20, 463-467.

Gustafson, R.I.; Albright, R.L.; Heisler, J.; Lirio, J.A., and Reid, O.T. Ind. Eng. Chem., Prod. Res, Dev, 1968, I, 107-115. 
Harker, H.; Gallagher, J.T.; and Parkin, A. Garbon, 1966, 4. 401-409.

Harker, H.; Jackson, C.; and Wynne-Jones, W.F.K. Proc, ROY, Sec. (Londonl, 1961, A262, 328-339.

Hochstein, F.A. I, Am, Chem, Soc, 1949, 71, 305-307.

Ingram, D.J.E. Dise, Earaday Soc, 1955, 19, 179-181.

Ingran, D.J.E.; Tapley, J.G.; Jackson, R.: Bond, R.L.: and Murnaghan, A.R. Mature, 1954, 174, 797-798.

Kim, B.R.; Snoeyink, V.L.; and Saunders, F.M. Journal WPCE, 1976, 48, 120-133.

Kipling, J.J. Adnorption from Solutions of Non-Electrolyten, Acadenic Press, NY, 1965. Krynitsky, J.A.: Johnson, J.s.; and Carhart, H.W. I. Ame Chem, Soc., 1948, 70, 486-489.

Kuo, Y., University of California, Berkeley, Personal Communication, 1983.

Magne, P. and Walker, P.I. Carbon, 1986, 24, 101-107. Mahajan, O.P.: Moreno-Castilla, C.; and Walker, P.L. Separation Sci, Tech,, 1980, 25, 1733-1752.

Mahajan, O.P.; Youssef, A.; and Walker, P.L. Separation Sci, Teche, 1978, 13, 487-499.

Mattson, J.S. and Mark, H.B. Activated Garbon, Marcel Dekker, NY, 1971. 
Mattson, J.S.; Mark, H.B.; Malbin, M.D.; Weber, W.J.; and Crittenden, J.C. I, colloid Interface Sci, 1969, 31, 116-130.

Modell, M.; deFilippi, R.; and Krukonis, V., "Regeneration of Activated Carbon with supercritical Carbon Dioxide", in Activated carbon Adsorption of organies from the Aqueous Phase, Volume 1, Suffet, I.H. and MeGuire, M.J., Eds., Ann Arbor Science Publishers, Inc., Ann Arbor, MI, 1980. Müller, G.: Radke, C.J.; and Prausnitz, J.M. I, Phys. cheme, 1980, 84, 369-376.

Munson, Ph.D. Dissertation in Cheaical Engineering, University of California, Berkeley, 1985.

Neeley, J.w. U.S. Patent $4040990,1977$.

Neeley, J.W. Carbon, 1981, 19, 27-36.

Paleos J. J, colloid Interface scie, 1969, 31, 7-18. Puri, B.R. Garbon, 1966, 4, 391-400.

Puri, B.R. "Carbon Adsorption of Pure Compounds and Mixtures From Solution Phase", in Activated carbon Adsorption of organice from the Aounous phase, volume 1 , Suffet, I.H. and McGuire, M.J., Eds., Ann Arbor Science Publishers, Ine., Ann Arbor, LiI, 1980.

Puri, B.R. "Surface Complexes on Carbons", in chemistry and Physice of Carbon, Vol, 6, Walker, P.L., Marcel Dekker, Ed., NY, 1970. 
Puri, B.R. and Bansal, R.C. Carbon, 1964, 1, 457-464. Polio, F.X. and Kunin, R. Environ, Sci, Technol, 1967, 1, $160-163$

Polysciences Inc. Polysciences Inc. 1986-1987 catalog, Warrington PA, 1985.

Reiss, G. and Donnet, J.B. "Colloque sur la Physio-chemie de Noir de Carbone, Nulhouse, 27-28 Septembre, 1963", in Rev. Gen, Caoutchouc Plastioues, Vol, 41 , Donnet, J.B., Ed., Parie, 1964.

Riven, D. Rubber Chomiftry and Iechnology, 1963, 36, $729-739$.

Rohm and Hass Corp., "Amberlite IRC-50", Philadelphia PA, 1977.

Schirmann, J.P. and Delavarenne, S.Y. Hydrogen Reroxide in organic chomiftry, edition et documentation industrielle, Paris, 1979.

Snoeyink, V.L.; Weber, W.J.; and Mark, H.B. Environ. Sci. Iechnol, 1969, 3, 918-926.

Suzuki, M.; Misic, D.M.: Koyama, O.; and Kawazoe, K. Chem. Eng, Sci, 1978, 33, 271-279.

Sucikno, T. and Himmelstein, J. Ind, Eng, Chem, Eundam., 1983, 22, 420-425.

van Vliet, B.M. and Weber, W.J. Journal WPCF, 1981, 53, 1585-1598. 
Waitz, W., Rohm and Haas Corp., Personal Communication, 1987.

Wojaczynska, M.: Kolarz, B.N.: and Rupicz, K. Reactive

Polymers, 1984, 3, 67-72.

Yonge, D.R.: Keinath, T.M.; Poznanska, K.; and Jiang, Z.P. Environ, Scie Technel., 1985, 29, 690-694. 
Chapter 5 oxidative Coupling as a Mechanism for Irreversible Adsorption

\subsection{Characteristics of Oxidntive coupling Renctions}

Many phenolic compounds are readily oxidized. The reaction products usually include dimers, as well as higher polymers and compounds that are quinoid in nature (Musso, 1967). In most cases, oxidation involves removing one hydrogen atom from each phenol molecule and joining the intermediates by carbon-carbon bonds at positions ortho and/or para to the hydroxyl groups. Iess erequently, carbon-oxygen linkages are formed.

Many coupling produces have low solubilities is Nater and in nompolar organic solvents, but are soluble in polar organic solvents. Iim et al. (1983) and Ficek and Boll (1980) obtained dark brown and black amorphous precipitates by oxidizing phenol in aqueous media. The precipitates had high aelting points and were highly soluble in dimethylformamide and dimethylsulsoxide (Iim et al., 1983). Ihis solubiitiz behavior is consistent with the presence of hydroxyl and quinoid groups in carbonaceous oxidation products. 
Several mechanisms have been proposed for phenol coupling reactions (see i.e. Barton, 1967), but it is generally believed that free radicals are involved (see i.e. Musso, 1967 and Nonhebel and Walton, 1974). Figure 5.1 illustrates possible pathways for coupling of phenol molecules in the presence of an initiator. The reaction is initiated with the formation of phenoxy radicals from phenol molecules or from phenolate anions. Initiators (denoted by 'In' in the figure) can take many forms, but are generally reduced during the reaction. Phenoxy radicals react irreversibly with phenoxy radicals, phenol molecules, or phenolate anions to form dimers. Electron localization in the radicals is responsible for the observed coupling positions fortho and para to hydoxyl groups) and for the predominance of carbon-carbon linkages. Further reactions of tha coupling products form polymers and quinoid compounds.

Aniline also undergoes oxidative coupling reactions that involve free radicals. It has been demonstrated that a radical containing bivalent nitrogen is formed in the oxidation of aniline (Fieser, 1930a). Nitrogen radicals generally polymerize to hydrazines rather than forming carbon-carbon linkages, however.

oxidative coupling of dihydroxybenzenes proceeds through other mechanisms (Nonbebel and Walton, 1974). semiquinone radical anions are formed from catechols 


\section{Initiation}

$$
\begin{aligned}
& \mathrm{In}+\mathrm{PhOH} \longrightarrow \mathrm{InH}+\mathrm{PhO} \cdot \\
& \text { or } \mathrm{In}+\mathrm{PhO}^{-} \longrightarrow \text { In }-\mathrm{PhO} .
\end{aligned}
$$

Irreversible Reaction

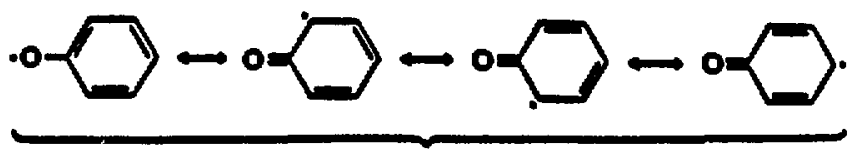

$+\mathrm{PhO} \cdot \mathrm{PhOH}$, or $\mathrm{PhO}^{-}$
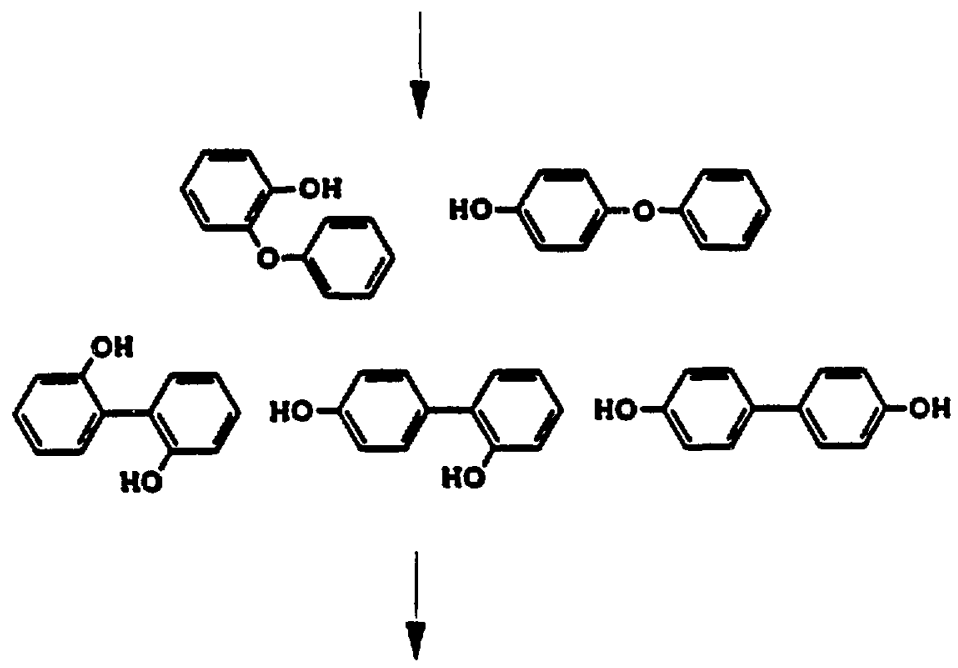

Polymers, Quinoids

Figure 5.1 Oxidatlve Coupling of Phenolic Molecules. 
and hydroquinones and are relatively stable compared to their monohydric counterparts. Semiquinone radical anions do not couple, but oxidize to form quinones. The quinones are readily attacked by nucleophiles such as the anions of catechol or hydroguinone, and so coupled products form. Semiquinone radicals formed from resorcinols cannot oxidize to form a quinone, and under anaerobic conditions give only carbon-carbon coupled dimers. When air is present hydroperoxy radicals torm, and dehydration leads to a quinone that can be attacked by nucleophiles.

\subsection{Consistency of the Renetion charneteristies with Experimental obeervations}

The concept of oxidative coupling of phenolic compounds during adsorption by activated carbon is consistent with most of the experimental observations discussed in this dissertation. Preliminarily, the effects of time and temperature on total and irreversible uptakes suggest that chemical reactions with appreciable activation energies occur. Lim et al. (1983) calculated an activation energy of $12.5 \mathrm{Kcal} / \mathrm{mol}$ for aerobic coupling of phenol in an alkaline media using the strong catalyst cuprous chloride. Activated carbon is presumably a much weaker catalyst than cuprous chloride, although the surface may play other roles in the coupling reactions as discussed in section 5.2.4, so higher activation energies might be expected for coupling 
reactions between sorbate molecules. Higher activation energies would also be expected in neutral and in acidic media, as discussed later in this section.

The occurrence of a high-activation-energy chemical reaction helps to explain why irreversible adsorption has been observed in some investigations, but not in others. With reference to Chapter 1, investigators using relatively high adsorption temperatures, relatively long adsorption contact times, and/or a drying step between adsorption and regeneration, saw evidence of irreversible adsorption. Suzuki et al. (1978), seewald et al. (1983), Yonge et al. (1985), and Feurstenau (1984) are in this category. In contrast, Zogorski and Faust (1978), Thakhar and Manes (1987), Robertaccio (1976), and in some trials seewald et al. (1983), used lower temperatures and/or shorter adsorption contact times and did not see irreversibilities. In the investigation by yonge et al., the divergence of isotherms was most likely due to differences in cumulative adsorption contact times.

\subsubsection{Desorbable Reaction Products}

Polymers desorbed from Columbia carbon in the experiment reported in section 4.5 are products of the oxidative coupling of phenol molecules. Because of their presumably low solubilities in heptane, the polymers were probably strongly adsorbed and were not detected through GC 
analysis of the heptane phase. The polymers were not detected by HPLC analysis of the legenerant, probably because of low aquecus phase solibilities. An aqueous mobile phase was used to elute compounds from the $C N$ column (see Appendix I). Consequently, these polymers were included in the irreversible uptake reported in section 4.5, even though they were removed from the carbon by acetone.

It is likely that desorbable reaction products $\because$ contributed to all irreversible uptakes reported in this work. Unfortunately, the discovery of these products was made very late in this investigation, so that the amounts of the products were not determined. The observations (made in section 4.3.2) that irreversible uptakes affected capacity only mildly and that there were substantial irreversible uptakes by regenerated carbon, suggest that the amounts of desorbable products may have been substantial.

The chromatograms in section 4.5 show that the higher the molecular weight of a polymer, the smaller the amount desorbed. Qualitatively, this result is consistent with the expected product distribution of an oxidative coupling reaction in a liquid phase. Further, the solubilities of the products probably decrease with increasing molecular weight, and, if so, higher molecular weight products wouid be more difficult to desorb. It may be beneficial, 
therefore, to employ a more polar solvent than acetone (i.e. dimethylformamide) in the regeneration of carbons. Finally, high molecular weight polymers could form and become trapped in carbon pores. Such entrapment is consistent with the observation of Seewald et al. (1983, see Chapter 1) and of Magne and Walker (1986) that chemisorption of phenol blocks the smallest pores.

As discussed in Chapter 1, seewald et al. identified two desorbable producte during the thermal regeneration of carbon loaded with phenol. One of the products, hydroxy-diphenylether is formed through oxidative coupling. The second product, dibenzofuran may be formed by dehydration of hydroxyediphenylether when the linkage is para to the hydroxyl group.

\subsection{2 sorbate Ranetivities}

organic compounds differ markedly in their tendencies to undergo oxidative coupling. In most cases, tise relative reactivities of sorbates on activated carbon can be rationalized in terms of their reactivities as solutes in coupling reactions.

\subsubsection{Nonrenctive Sorbates}

Several sorbates wex id essentially nonreactive under the conditions used for adsorption. Alkanes and nonaromatic alcohols are in this category, as indicated by the classification of organic adsorbates by suzuki, et al. 
(1978; see Chapter 1, Table 1.2). These compounds are much more difficult to oxidize than phenols, because intermediates are not resonance stabalized.

other compounds that do not seem to react on carbon include 5,5-dimethylhydantoin and butyric acidl. The absence of aromatic rings in these compounds makes them more difficult to oxidize than are phenols.

The experimental results summarized in section 4.2 .1 and in Table 1.2 also indicate that many benzenes are nonreactive sorbates. Benzenes are generally much more difficult to oxidize than phenols that have the same substituents, and benzene, p-xylene, anisole, benzaldehyde, and nitrobenzene did not react on carbon surfaces while phenol, 2,4-dimethylphenol, p-methoxyphenol, p-hydroxybenzaldehyde, and p-nitrophenol did. Toluene and counarin were also nonreactive.

In contrast, the results of suzuki et al. (1978) show that benzoic acid was difficult to Iemove from carbon through thermal regeneration. "Aromatic compounds tend to be more easily oxidized than benzoic acid" (Nonhebel and walton, $1974, p .419)^{2}$, so this result seems to be at

1 Evidence to support this statement can be found in Sections 2.4 and 4.2 .1 , and in Table 1.2 .

2 Further, oxidative coupling is more facile for phenol than for p-hydroxybenzoic acid (Feiser, 1930). One might expect, then, that it is more difficult to oxidize the aromatic ring of benzoic acid than it is to oxidize benzene. 
variance with the correlation between oxidative coupling and sorbate reactivities. Benzoic acid has a higher boiling point than the other benzenes tested by Suzuki et al., however, and may have decomposed on the surface before it was volatilized. Furthar tests are needed on the adsorption and recovery of benzoic acid before this result can be fully underetood.

\subsubsection{Hydroxybensenes and aniline}

Table 1.2 and the renulte reported in section 4.2 indicate that hydroxybenzenes and aniline are very reactive on carbon surfaces. There is a semiquantitative correlation between sorbate reactivities and reactivities of the same compounds in oxidative coupling reactions.

Semiquantitative orderings of sorbate reactivities were developed in section 4.2.5. Table 4.19 summarizes the comparisons of irreversible uptakes at solute activities of 0.002 (mole fraction) and at total uptakes of 3.0 micromole $/ \mathrm{m}^{2}$. The two methods of comparison gave similar results.

Reactivities of these compound in oxidative coupling reactions ean be characterized by 'critical oxidation potentials' (COPs) whith are listed in Table 5.1. Fieser (1930ab) tested phenols and amines with oxidizing agents of known redox potentials in order to determine the potential that was just sufficient to oxidize each compound in an 
Table 5.1 Critical Oxidation Potentials of Phenolic Compounds and Aniline

\begin{tabular}{ll} 
Compound & COP (v) \\
\hline p-Methoxyphenol & 0.848 \\
2,4-Dimethylohenol & 0.895 \\
Phenol & 1.089 \\
p-Chlorophenol & 1.094 \\
Aniline & 1.135 \\
p-Hydroxybenzaldekyde & 1.301 \\
p-Nitrophenol & 1.433 \\
\hline
\end{tabular}


aqueous-alcohol mixture at $25^{\circ} \mathrm{C}^{3}$. The COPs are not precise redox potentials, but they do provide a measure of the relative ease of oxidation of the compounds tested. In addition, Fieser established approximate values for the influence of ortho and para substituents on the COPs of phenols and aromatic amines. Since sorbate reactivities were monitored under different conditions than those used to establish COPs, and since several COP values listed in Table 5.1 were calculated using the approximate values that Fieser established for substituents, Cops serve only as a seaiquantitative guide to reactivities expected for coupling reactions on carbon.

The concept of critical oxidation potentials was established because it was not possible to determine true redox potentials for phenolic compounds and aromatic amines with standard titration methods (Musso, 1963). Oxidative coupling reactions are irreversible and are generally too rapid for that experimental technique. Polargraphic analysis could be used to establish relative oxidation potentials (Musso, 1963), but data could not be located for some of the compounds tested in this work.

There is a linear relationship between COPs and the theo:etical energy valies for the highest occupied phenol

3 Therefore, the lower the COP, the more reactive the compound. 
orbitals as calculated by the LCAO-MO method ${ }^{4}$. This fact led Fueno, et al. (1959) to conclude that in each oxidation the rate determining step involves abstraction of one electron from the highest occupied orbital. Thus, the easier it is to form a radical, the faster the reaction should be.

Irreversible uptakes reported in the second and third columns of Table 4.19 are plotted against critical oxidation potentials in Figures $5.2 a$ and b respectively ${ }^{5}$. In each figure, there is a reasonable correlation between the variables. Given the crude nature of the reactivity scales, these correlations lend strong support to the contention that oxidative coupling is involved in the irreversible adsorption of phenolic compounds.

The regeneration results of Yonge et al. summarized in Table 1.3 are partially consistent with the observed corelation between sorbate reactivities and COPs. The cOPs of phenol, o-cresol, and o-methoxyphenol are 1.089, 1.040, and 0.868 respectively, and the lower the COP, the greater the percent irreversibility. ortho-positioned alkyl substituents affected percent irreversibilities more than did a para-positioned alkyl substituent, however, and there is not a parallel trand with COps (see Fieser, 1930a).

\section{- Linear combination of atomic orbitals - molecular orbitals}

5 The two connected symbols in Figure $5.2 \mathrm{~b}$ represent the range of irreversible uptakes for phenol that is listed in the third column of Table 4.19. 


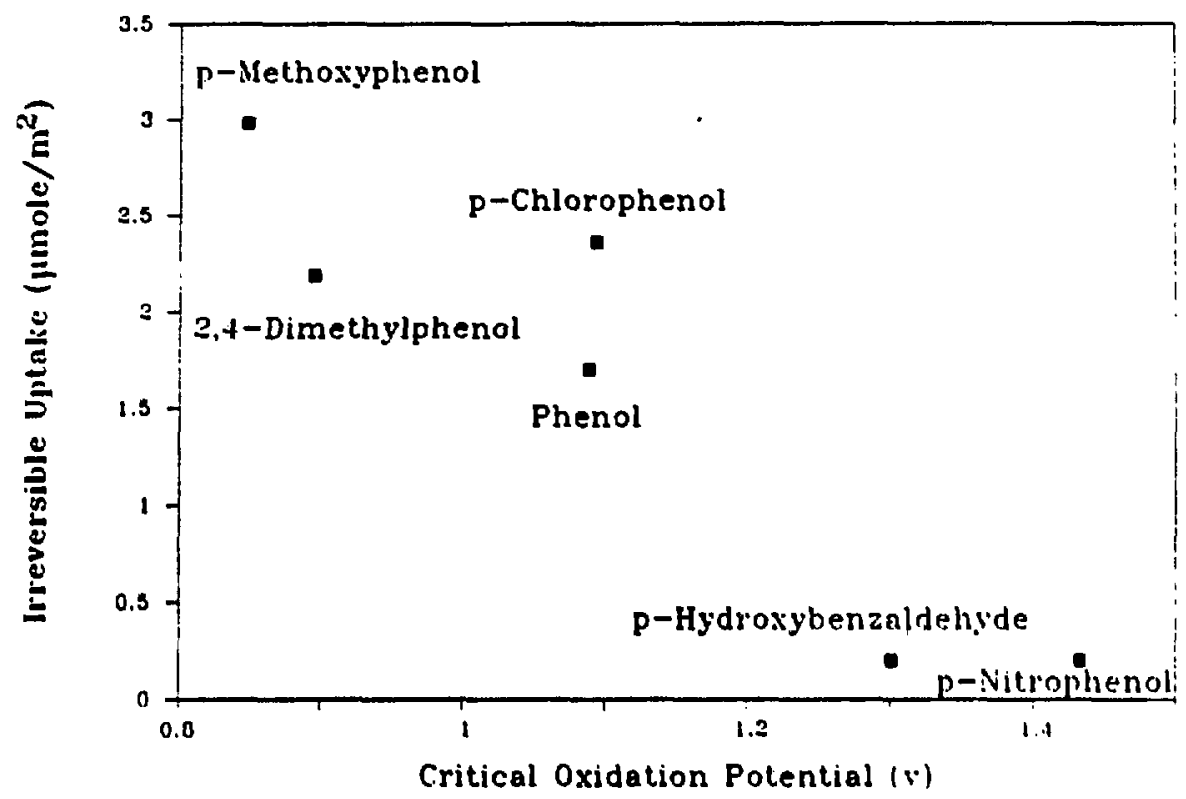

Figurc 5.2a Estimated Amount of Irreverslble Adsorptlon onto Columbia Carbon at Solute Activity $=0.002$ (Mole Fraction) vs. Critical Oxidatition Potentlal for Pheaollc Componnds. 


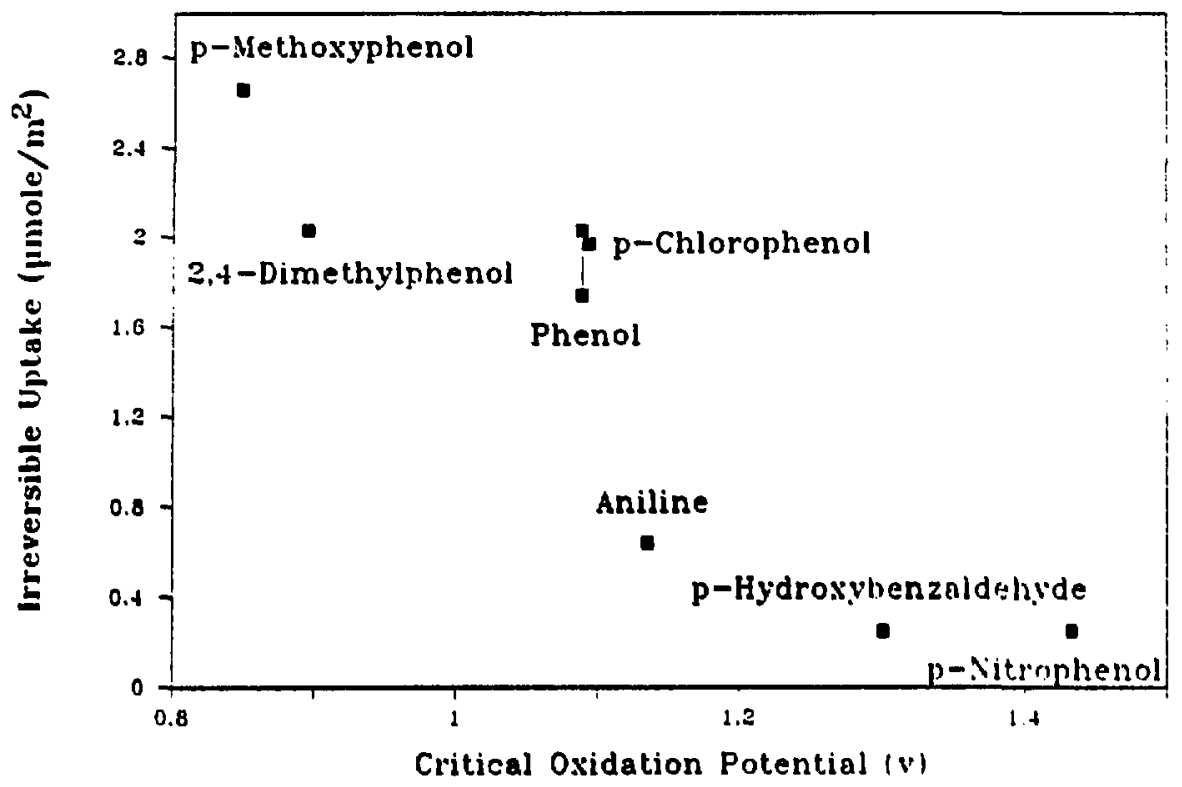

Figure 5.2b Estimated Amount of Irreversible Adsorption onto Columbia Carbon at Total Adsorption $=3.0 \mu \mathrm{mole} / \mathrm{m}^{2}$ vs. Crifical Oxidatitlon Potentlal for Phenolic Compounds and Aniline. 
Critical oxidation potentials can also be used to show that dihydroxybenzenes are more reactive than monohydroxybenzenes, but care must be taken in making the comparison. The oxidation potentials for those dihydroxybenzenes which can form quinones directly from semiquinone radicals reflect the energy required to remove two electrons and are lower than the potentials needed to remove the first eiectron. Fieser (1930a) relates cops to free energies of oxidation, and his calculations show that hydroquinone and catechol are much more readily oxidized than is phenol.

Resorcinol cannot form a quinone directly from a semiquinone radical, and, consequently, its $\operatorname{cop}$ is not a great deal lower than that of phenol $(1.043 \mathrm{v}$ vs $1.089 \mathrm{v})$. This fact indicates that the differences in interactions of these compounds with F-100 (discussed in sections 2.3 and 2.4) may not be due solely to oxidative coupling reactions. It is possible, however, that oxygen associated with the carbon surface enhances the reactivity of resorcinol more than it enhances the reactivity of phenol. As noted earlier, semiquinone radicals derived from resorcinol can form more reactive intermediates in the presence of oxygen.

\subsubsection{Iiquid Phase Properties}

As demonstrated in section 4.4 , the nature of the liquid phase can greatly influence uptakes of phenolic 
compounds by activated carbons. This section draws parallels between those influences and influences of liquid phase properties on oxidative coupling reactions. Since sorbate molecules react at the adsorbent surface, the influences of liquid phase properties on the characteristics of the surface layer are of primary importance.

\subsubsection{1 a Potential Role of Molecular oxygen}

Molecular oxygen acts as an initiator in oxidative coupling reactions (Denisov, 1980). Interaction between $\mathrm{O}_{2}$ and phenolic sorbates may be direct, or, as discussed in section 5.4, may involve a catalyet and may be indirect. In water, oxygen attacks both phenol molecules and phenolate anions forming $\mathrm{HO}_{2}{ }^{*}$ and $\mathrm{O}_{2}{ }^{-}$- radicals along with phenoxy radicals. Subsequent reactions include (Shibaeva et al., 1969):

$$
\begin{gathered}
\mathrm{O}_{2}^{+}+\mathrm{H}^{*}: \mathrm{HO}_{2} \\
\mathrm{PhOH}+\mathrm{HO}_{2} \rightarrow \mathrm{H}_{2} \mathrm{O}_{2}+\mathrm{PhO} . \\
\mathrm{PhOH}+\mathrm{H}_{2} \mathrm{O}_{2} \rightarrow \mathrm{PhO}+\mathrm{H}_{2} \mathrm{O}+\mathrm{HO}^{\circ} \\
\mathrm{PhOH}+\mathrm{HO}^{\circ} \rightarrow \mathrm{PhO}+\mathrm{H}_{2} \mathrm{O}
\end{gathered}
$$


as well as phenol coupling reactions. Through these reactions, four phenoxy radicals can be formed for each molecule of oxygen consumed.

The experimental results discussed in section 4.4 .3 are consistent with the ability of molecular oxygen to promote oxidative coupling reactions. since oxygen is consumed in the oxidation process, oxygen initially in the headspace of a vessel could influence irreversible uptakes. Phenoxy radicals can be formed through the actions of initiators other than oxygen, however, so excluding oxygen from the IIquid and gas phases during an adsorption contact does not preclude oxidative coupling reactions. It is also possible that molecular oxygen adsorbed on carbons prior to an adsorption contact would promote oxidative coupling even when no oxygen was present in a fluid phase.

\section{2 .3 .2 The Influence of $\mathrm{pt}$}

oxidative coupling of phenolic compounds is more facile in alkaline than in acidic media. Penketh (1957) used a polarographic method to show that oxidation potentials of several phenolic and amino compounds decreased linearly with increasing pH values in buffered aqueous-alcohol solutions. Further, Ieaction of phenolic anions with oxygen is much faster than is reaction of the corresponding phenols (Denisov, 1980; Shibavea et al, 1969). Consistently, Chin et al. (1985) found that the rate of 
aerobic coupling of phenol on a cuprous chloride catalyst had a second-order dependence on the solution $\mathrm{pH}$ when the $\mathrm{pH}$ values were below the $\mathrm{pk}_{\mathrm{a}}$ value. These facts indicate that phenoxy radicals are formed much more readily from anions than from molecular compounds.

The experimental observations made in section 4.4 .2 are generally consistent with an oxidative coupling mechanism for irreversible adsorption. The dopendencies of oxidation potentials and coupling reaction rates on solution pH parallel the adsorption results. Specifically, irreversible uptakes by columbia carbon decreased with decreasing pH value, below the $\mathrm{pKa}_{\mathrm{a}}$ of phenol. Too, almost all of the adsorption was irreversible at $\mathrm{pH} 12.1$.

The degree of ionization of a sorbate does not seem to be the primary variable determing its reactivity on carbon, however. If it were, the lower the $\mathrm{pK}_{\mathrm{a}}$ value of a sorbate, the more reactive it should be in neutral aqueous solutions. As shown in Table 4.19, sorbates with lower $\mathrm{pKa}_{\mathrm{a}}$ ialues were generally less reactive than sorbates with higher $\mathrm{pK}_{\mathrm{a}}$ values. Relative reactivities were in line with critical oxidation potentials determined for unionized compounds.

\subsubsection{Ioptane Conpared to Jater}

Phenols are unionized in nonpolar solvents, so oxidative coupling reactions are less facile than they are 
in ionizing media. In particular, oxidation with $\mathrm{O}_{2}$ is faster in water than in hydrocarbon mixtures (Denisov, 1980). Even though the fraction of phenol molecules that are ionized in a neutral aqueous solution is only $1 \times 10^{-3}$, reaction rates can be several orders of magnitude higher for phenolate anions than for phenol molecules (Shibaeva et al., 1969). Consequently, oxidative coupling of phenol molecules should be more facile in neutral aqueous solutions than in heptane solutions.

The comparisons made in section 4.4 .2 between uptakes of phenol fros water and from heptane are in line with this reasoning. Those comparisons showed that at the same liquid phase activities, irreversible uptakes of phenol from heptane were lower than those from water. The issue is complicated by the fact that, in most cases, reversible uptakes were also lower from heptane than from water. Lower surface concentrations could result in lower rates for reactions on the carbon surface 6 .

\section{2 .4 Adsorbent Properties}

Many studies have shown that activated carbons can catalyze oxidation reactions (Garten and Weiss, 1957). These studies show not only that metallic elements in ash can catalyze oxidation of sorbates, but also that carbon

- The activities and surface coverages coinpared in section 4.4.1 are well below the values corresponding to the internal maximum in irreversible adsorption that appears in Figure 4.14 . 
surfaces themselves can be catalytic. While the mechanism of oxidation by carbons is open to speculation, it has been shown that the presence of molecular oxygen and the nature of surface oxides influence reaction rates (Epstein et al., 1971; Garten and Weiss, 1957).

Garten and Weiss (1957) have suggested a mechanism that accounts for the observed effects and is consistent with oxidative coupling of phenolic compounds on carbon surfacas. They propose that molecular oxygen can add to olefinic bonds associated with quinone groups to form peroxides. A peroxide group could then accept an electron from a quinone group, resulting in a monovalert peroxide anion radical and a semiquinone (radical). The peroxide radical could then accept a second electron from a second quinone group and split off as a peroxide anion. In a protic solvent, the peroxide anions would protonate yielding hydrogen peroxide.

This mechanism has similarities with that discussed in section 5.2.3.1. In particular, several phenoxy radicals can form for each molecule of oxygen consumed, since two radicals form on the carbon surface and since hydrogen peroxide is created. If the radicals abstract hydrogen atoms from sorbate molecules, four sorbate radicals could result. Both mechanisms are consistent with the experimental results discussed in section 4.4 .3 , but the mechanism suggested by Garten and Weiss can be used to 
explain why some surfaces promote oxidative coupling and others do not. Further, both mechanisms could lead to more irreversible adsorption than could be accounted for by $1: 1$ reactions with surface functional groups.

Radicals formed on the carbon surface could also couple directly with sorbate molecules, and sorbate radicals could couple with the aromatic surface. The sorbate would then be covalently bonded to the carbon and could not be removed by solvent regeneration. Thus, carbon could be a reactant as well as a catalyst in oxidative coupling reactions.

The mechanism suggested by Garten and weiss for the reduction of molecular oxygen depends on the ability of the carbon to form semiquinones and, hence, would be most facile in alkaline media (Garten and Weiss, 1957). This fact is consistent with the observed influence of pH on irreversible uptakes of phenolic compounds (see section 4.4.2).

The FTIR analyses discussed in section 4.1.3.3 indicated quinone functionality on witco columbia and on Norit Row $0.8 \mathrm{~s}$. Therefore these carbons could presumably oxidize phenolic compounds according to the suggested mechanism. It is not known whether Filtrasorb-100, Jitcarb 950, and/or Amoco Super Sorb have quinone functionality.

oxidation of Columbia carbon could interfere with the suggested mechanism. In general terms, an oxidized carbon 
should be less of a reducing agent for molecular oxygen than is the parent carbon. More specifically, oxidized Columbia has more surface funtional groups than does Columbia (see Section 4.1.4.3), and presumably, would have fewer reactive olefini = bonds. The observation made in section 4.1 .4 that the abundance of surface oxides correlates with the ragenerability of samples of oxidized Columbia is consistent with the idea that oxidation decreases the availability of sites at which peroxides can form. Secondarily, it is possible that quinone groups are destroyed by some oxidative treatments. In either case, it is possible to reconcile decreased irreversible uptakes by oxidized carbons with decreased abilities of those carbons to promote the oxidation of sorbate molecules.

The carbonaceous adsorbents $X E-340$ and $X E-348$ promote irreversible adsorption of phenol, but probably do not have quinone functionality. It is probable that sulfur-containing functional groups such as sulfoxides can donate electrons to affect the reduction of molecular oxygen. Sulfur-containing groups in XE-348 are probably in a lower oxidation state than those in XE-340 (see Neeley, 1981), and as discussed in section 4.1.5, XE-348 promotes irreversible adsorption of phenol more than does XE-340. Hence, a mechanism similar to that suggested by Garten and Weiss could account for irreversible adsc .tion of phenol by these carbonaceous adsorbents. 
The fact that the phenol-formaldehyde resin s-761 promotes irreversible adsorption is more difficult to rationalize. One possibility is that the resin became oxidized aric then it reacted with sorbate molecules. The resin is reported to be stable at temperatures up to $80^{\circ} \mathrm{C}$ in neutral, nonoxidizing media, but softens and swells in alkaline media and in the presence of oxidants (Diamond Shamrock Corp., 1978). The adsorption conditions used for the tests reported in section 4.1 .4 .5 might be considered 'borderline,' since oxygen was present in neutral pH mixtures that were hold at $80^{\circ} \mathrm{C}$ for an extended time. Alternatively, phenolic groups on the resin might catalyze reactions between sorbate molecules, but the experimental results reported in section 4.1 .4 .3 indicate that phenolic groups added to Columbia carbon did not promote reactions.

The resins XAD-4, XAD-7, and IRC-50 are all resistant to oxidation (Wajt.2, 1987; Rohm and Haas Corp., 1977), and therefore they should not promote oxidation of jmenolic sorbates. This conclusion is consistent with the experinental results reported in sections 4.1 .1 and 4.1 .4 .5 .

\section{2 .5 sunnary}

In summary, the concept that oxidative coupling of phenolic compounds on carbon surfaces leads to irreversible adsorption of the products, is consistent with many 
experimental observations. Coupling products have beti: identified in regeneration meaia, sorbate reactivities correlate with critical oxidation potentials, and liquid-phase properties that promote oxidative coupling also promote irreversi" " adsorption. Fucther, while the role of the adsorbent in oxidative coluling is speculative, carbon can catalyze oxidation rea $\%$

\subsection{References}

Barton, D.H.R., "Foreword", in Oxidative soupling ot Blyenols, Taylor, W.I. and Battersby A.R., Eds, Marcel Dekker, NY, 1967.

Chin, K.C.; Cha, J.A.: and Eim, P.K. Inde Eng, cheme Procese Dea, Dey, 1985, 24, 339-343.

Denisov, E.T., "The oxidation of alcohols, ketones, athers, esters, and acids in solution", in comprehensive chexical Kineties, Vol, 16, Lieuid-Phase Oxidation, Bamford, C.H. and Tipper, C.H., Eds, Elsevier Scientific, Amsterdam, 1980 .

Diamond Shamrock Corp., "Duolite s-761 Resinous Adsorbent", Redwood City CA, 1978.

Epstein, B.D.; Dalle-Molle, E.; and Mattson, J.B. Carbon; 1971, 2, 609-615.

Ficek, K.J. and Boll, J.E. Aqua (Iondonl, 1980, I, 153-156. Fieser, L.F. I, Amer, Shem, Soc., 1930a, 52, 5204-5201. 
Fieser, L.F. I, Amer, Chem, Soc, 1930b, 52, $4915-4940$. Fueno, T.: Iee, T.; and Eyring, H. I. Phys, Chems,, 1959, 63, $1940-1948$.

Fuerstenau, D.W. Snminar on the surface Propertief of coal and It's Flotation, Technical University of clausthal, West Germany, June, 1984.

Garten, V.A. and Weiss, D.E. Rey, Pure Apol, Chem, 1957, 2. 69-122.

Iin, P.X.: Cha, J.X.: and Patel, C.P. Ind, Eno, Chom. Procens Dafe Dext, 1983, 22, 477-482.

Magre, P. and Walker, P.I. Crebon, 1986, 24, 101-107.

Musso, H., Anowt, Chen, Internat, Edite, 1963, 2, 723-735. Musso, H., "Phenol Coupling", in oxidntive coupling of Bhenols, Taylor, N.I. and Battersby A.R., Eds, Marcel Dekker, KY, 1967.

Necley, J.H. Carbon, 1981, 19, 27*36.

Wonhwbel, D.C., and Walton, J.C. Eren-radical Chemistry, cambidge University prese, canbridge, 1974.

Penketh, G.E. I, Apol, Chen, 1957, I, 512-521.

Robertaccio, F.I., Ph.D. Thesis in Environnental Engineering, University of Deleware, 1976.

Rohn and Hass Corp., "Amberlite IRC-50", Philadelphia PA, 1977. 
Seewald, H.; Klein, J.; and Juntgen, H., "Correlation Between Desorption Behavior and Adsorption Characteristics of Organic Substances on Activated Carbon". Fundamentals of Adsoration: Procedings of the Engineering Foundation conference held at Schlose Elmav, Bavaria, West Germany, Yay 6-12, 1983, eds. Meyers, A.L., and Belfort, G., Engineering Foundation, New York, 557-565, 1983.

Shibaeva, L.V.; Metelitsa, D.I.; and Denisov, E.T. Kinetias and Gatalysis, 1969,10 , No. 5, 832-836.

Suzuki, K.: Kisic, D.K.; Koyama, O.: and Kawazoe, K. chem, Enc, sei, 1978, 33, 271-279.

Thakkar, S. and Manes, M. Environ, Sci, Technele, 1987, 21, 546-549.

Yonge, D.R.; Keinath, T.M.; Poznanska, K.; and Jiang, Z.P. Environ, Sci, Technole, 1985, 12, 690-694.

Waitz, W., Rohm and Haas Corp., Personal Communication, 1987.

Zogorski, J.S. and Faust, S.D., "Equilibria of Adsorption of Phenols by Grenular Activated Carbon", Chemistry of Wasterater Technology, ed. Rubin, A.J., Ann Arbor science Publishers, ann Arbor, Mich, 143-160, 1978. 


\section{Chapter 6 sumary and Proeess Implications}

Phenolic compounds may be irreversibly adsorbed by activated carbons. Total uptakes increase with time and with increasing temperature, at rates that may be much lower than those for physisorption. Regeneration of carbons with acetone recovered at least as much phenol as did other solvents and displacers, and hence was used to define irreversible adsorption operationally.

The physiochemical properties of an adsorbent influence irreversible uptakes. Carbonaceous adsorbents and a phenol-formaldehyde resin (S-761) promoted irreversible adsorption, while a styrene-divinylbenzene copolymer (XAD-4), an acrylic ester-divinylbenzene copolymer (XAD-7), and a methacrylic acid-divinylbenzene based cation exchanger (IRC-50, carboxylic acid functionality) did not. oxidation of Witco columbia carbon with nitric acid or with hydrogen peroxide resulted in lower total and irreversible uptakes of phenol fron water. Columbia carbon reduced with lithium aluminum hydride had the same capacity for phenol, but was more regenerable than the untreated carbon. Columbia carbon treated with sulfur dioxide at $510^{\circ} \mathrm{C}$ and Columbia carbon treated with nitric acid and then ammonia at $600^{\circ} \mathrm{C}$ adsorbed less phenol from water and were more 
regenerable than was the untreated carbon. The abundance rather than the identities of surface functional groups on Columbia carbon seemed to have the greatest influence on total and irreversible uptakes.

Mineral ash in carbons can promote irreversibilities, but irreversible adsorption also occurs for materials containing little or no ash. Columbia carbon oxidized with $\mathrm{KMnO}_{4}$ retained manganese oxides and promoted irreversible adsorption to a greater extent than did the untreated carbon. In contrast, the carbonaceous adsorbents XE-340 and $X E-348$ and the resin S-761 promote irreversible adsorption, but contain little or no ash.

Unpaired electrons on the surface of an adsorbent prior to an adsorption contact are probably not the primary cause of irreversible adsorption. While EPR measurements showed that XE-348 contained fewer unpaired electrons than did $X E-340, X E-348$ caused more irreversible adsorption of phenol than did XE-340.

Sorbates differ markedly in their tendencies to undergo irreversible adsorption. Non-phenolic compounds such as benzenes are less reactive than monohydroxybenzenes, and monohydroxybenzenes are less reactive than dihydroxybenzenes. Aniline is reactive, but less so than phenol. There is a semiquantitative correlation between sorbate reactivities and critical oxidation potentials. 
Irreversible uptakes of phenol on Columbia carbon vary with solute activity in a complex manner. For activities at which there are low surface coverages, irreversible uptakes increase with increasing solute activity. This trend reverses for higher surface coverages. These facts may reflect differences in orientation of sorbate molecules at different surface coverages.

Irreversible and total uptakes of phenol on F-100 decreased with increasing cycle number during repetitive loading and regenoration of the carbon. Given the adsorption amounts, however, the decreases were small.

The properties of the liquid-phase can greatly influence uptakes of phenolic compounds by activated carbons. At the same liquid phase activities, irreversible uptakes of phenol from heptane were generally lower than those from water. Further, alkaline media and dissolved oxygen promoted irreversible adsorption of phenol.

Polymers of composition $\mathrm{C}_{6 n} \mathrm{H}_{4 n+2} \mathrm{O}_{n}$ ( $n=2$ to 7 ) were identified in acetone used to recover phenol from columbia carbon. The higher the molecular weight of of a polymer, the less abundant it was. The polymers formed during adsorption of phenol from heptane. Solvents that are more polar than acetone (i.e. dimethylformamide) may be more effective than acetone in removing phenolic polymers from carbons. 
These experimental observations and many made in previous investigations are consistent with an oxidative coupling mechanism for the irreversible adsorption of phenolic compounds by carbons. In particular, irreversibla uptakes are greater for sorbates that are readily oxidized. Further, conditions that favor oxidation of sorbates also favor irreversible adsorption. Finally, carbon can catalyze oxidation reactions, and coupling products have been identified in a regenerant mixture.

These lindings suggest meane of mitigating irreveraibilities in induntrial applications of activated carbon beds. For example, reduced bed volumes and rapid cycling times should be used to limit the residence time of phenolic compounds on carbon. In treatment schemes involving several processing uteps, it may also be possible to gain favorable adsorption conditions through judicious sequencing of those steps. When possible, adsorption and regeneration should occur at low temperatures and alkaline conditions should be avoided. These considerations favor the use of organic solvents for regeneration over thermal methods or the use of inorganic bases. Reagents that promote oxidation (including molecular oxygen) should also be avoided when possible. High solute activities may also lessen the extent of irreversible adsorption. Finally, it may be desirable to use carbon that has been oxidized under 
mild conditions. While oxidation generally reduces the capacities of carbons for phenol, it seems to improve regenerabilities. 


\section{Appendix I Chromatographic Methods}

Gas chromatography (GC) and high performance liquid chromatography (HPLC) were used to establish solute concentrations in adsorption and regeneration trials. The choice of method for a particular analysis was based on the ability to achieve a complete separation of components of a mixture, the sensitivity of the detector to those components, and the availability of the hardware. Often the desired separation could be achieved by either method, but lower solute concentrations could be measured using GC with flame-ionization detection. Details of the chromatographic methods are given below.

\section{Gas Chronatography}

A varian Associates 3700 Gas Chromatograph with flash injection and flame-ionization detection was used for GC analyses. Most often, the column was a 5-foot long 1/8-inch-0.d., 26-gauge stainless steel tube packed with Waters Associates Porapak-Q particles (100 x 120 mesh). These particles are porous polymer beads with polar monomers incorporated into the basic polymer structure. occasionally, a shorter column was used to achieve more rapid elution of compounds. One uliter samples were 
injected into nitrogen carrier gas (the mobile phase) and peak areas were compared to those of calibration samples in order to establish concentrations.

\section{High Performance Liquid Chromatography}

The hardware used for the HPLC analysis was a hybrid. A Perkin Elmer Series 10 Liquid Chromatograph provided flow control. Twenty pliter injections were made with a Rheodyne Syringe-Loading sample Injector (Model 7125-075). Detection was accomplished with a waters Associates Model 400 Absorbance Detector with an Extended Wavelength Module. A fixed wavelength of $214 \mathrm{~nm}$ (2ine source) was used in the module.

The analyses employed isocratic reversed-phase chromatography, Usually, the stationary phase was a $C_{18}$ Radial-Pak $\mu$ Bondapak column with $8 \mathrm{~mm}$ internal diameter, 10 cm length, and $10 \mu \mathrm{m}$ irregular particles. Radial compression of the column with a waters Associates z-module enhanced the separation efficiency. Occasionally, a Waters Associates $\mathrm{CN} \mu$ Bondapak steel column was used. This column has 9 percent CN group bonded to $\mu$ Porasil packing material and is more polar than a $C_{18}$ column. Both columns provide substrates that facillitate the separation of non-ionized solutes in a polar mobile phase. The mobile phase was methanol, water, or a mixture of methanol and water. 
Appendix II Thermodyamic Condsiderations

Nornalizing Adosrption Isothermo with Aetivitied

The Lundelius rule holds that there is an inverse relationship between the adsorption of a species and its solubility in the solvent used (Jaycock and Parfitt, 1981 ). The stronger the solvent-solute interactions, the smaller the amount of adsorption. Traube found that "The adsorption of organic substances (at the liquid-vapor interface) from aqueous solutions increases strongly and regularly as we ascend the homologous series"l, and this statement is known as Traube's rule (Adamson, 1982). Hansen and craig (1954) found that these rules took a quantitative form for homogolous series of acids and alcohols adsorbed from water onto carbon. For each series:

$$
K_{p 1} C_{n}=\text { constant }
$$

where $\mathrm{K}_{\mathrm{pi}}$ is the surface-solution partition coefficient and $c_{s i}$ is the solubility of solute $i$. That $i s$, isotherms

1 This is a statement by Freundlich (1926). 
within a homogolous series were nearly coincident when adsorption (in moles per gram of carbon) was plotted against solute concentration divided by solubility.

Equation II-I is an empirical relationship that holds only under particular conditions. The relationship is often discussed in the context of an idealized partitioning at a solid-liquid interfaci (Adamson, 1982; Jaycock and Parfitt, 1981). For two liquid phase components with equal surface phase activity coefficients and equal areas occupied by adsorbed molecules:

$$
K=\frac{x_{2} a_{1}}{x_{1}^{2} a_{2}^{l}}
$$

The symbol $\mathrm{K}$ is an equilibrium constant, $x_{i}$ is the mole fraction of $i$ in the surface phase and $a_{i} l$ is the activity of $i$ in the liquid phase. For $K$ to have approximately the same value for different members of a homologous series, activities must be defined in the Raoult's law sense rather than in the Henry's law sense. In dilute solutions the solvent activity $\left(a_{2}{ }^{2}\right)$ is virtually constant and equation II-2 can be rearranged to yield;

$$
\frac{x_{i}}{x_{i}} \cdot \frac{1}{f_{1}}=\text { constant }
$$


where $f_{1}$ is the activity coefficient for the solute in the liquid phase.

Equation II-1 is equivalen: to equation II-3 when the solubility of the solute equals the inverse of the activity coefficient. The equality does not always hold, and in particular, does not hold when the standard-state fugacity of a solid is taken as the fugacity of a hypothetical pure sub-cooled liquid (Prausnitz, 1969). In addition, the value of the activity coefficient is in general a function of the solute mole fraction. Equation II-3, then, is somewhat more general than is equation II-1, even though neither equation is rigorous.

Equation II-3 suggests that comparing adsorption isotherms plotted versus activity may be revealing. The use of activities defined in the Raoult's law sense normalizes the effects of solute-solvent interactions so that differences in amounts of adsorption can be attributed to differences in energies of interfacial interactions. This type of comparison is used in section 2.5 to support the contention that higher temperatures promote chemical reaction of phenol on activated carbon. Most isotherms in the present work are plotted on an activity basis to facilitate comparisons of surface interactions. 


\section{nctivity coefficients}

Activity coefficients used in this work are estimated from published values or from solubilities. The coefficients are defined in the Raoult's law sense. In most of the experiments reported, the solutions were extremely dilute, and a single value of the activity coefficient is used for all concentrations of a solute. This value is usually derived from data for a mixture with the solute at high dilution, but is refered to as an "infinite dilution" activity coefficient. Unless otherwise specified, the variation of activity coefficients with temperature is assumed to follow the relationship;

$$
\ln f^{*}=\frac{A}{T}+B
$$

as recemmended by Reid et al. (1987). In cases where an activity coeficient is available (or is estimated) at only one temperature, B is set equal to zero².

Infinite dilution activity coefficients used in tnis work are listed in Tajle II-1. The mathods used to determine these coefficients are summarized below.

2 Setting B $=$ implies that a solution is regular. This iz probably a poor assumption for phenolic solutes, but it usit to obtain reasonabie estimates. 
Table II-1 Infinite bilution Activity Coefficients for Aromatic Solutes

\begin{tabular}{lccc} 
Solute & Solvent & Temperature ${ }^{\circ} \mathrm{C}$ & $f_{1}$ \\
\hline Aniline & Water & 80 & 104 \\
Anisole & - & 80 & 218 \\
Benzene & - & 25 & 2504 \\
p-Chlorophenol & - & 80 & 1820 \\
2,4-Dimethylphenol & - & 80 & 99.8 \\
Hydroquinone & - & 80 & 76.8 \\
p-Hydroxybenzaldehyde & - & 25 & 4.29 \\
p-Methoxyphenol & - & 80 & 206 \\
Nitrobenzene & - & 80 & 77.2 \\
p-Nitrophenol & - & 80 & 2012 \\
Phenol & $:$ & 25 & 50.6 \\
& - & 53 & 63.5 \\
& Heptane & 25 & 55.2 \\
Resorcinol & - & 80 & 20.8 \\
\hline
\end{tabular}


Phenol in water

Extensive vapor-liquid equilibrium data are available for aqueous solutions of phenol in the temperature range 15-1000C (Markuzin, 1961: Schulek et al., 1964: Bogart et al., 1948; Weller et al., 1963; and Shreinemakers, 1900). Tsonopolous ( 1970 ) calculated activity coefficients at "practically infinite dilution" using data from these sources and equation 3.9 (rearranged). The values he calculated fron the data in the first four references are plotted in Figure II-2. Cohling (2977, 2981) analyzed the data of Schreineakers with the NRTL equation and the data of weller et al. with the UnIQUac equation, and calculated the remaining values in Figure II-1. The activity coefficients calculated by Gehling should be nore accurate than those calculated by rsonopolous erom the same data.

The equation

$$
\ln f^{*}=-6.3588+6.0273 \times 10^{3} \frac{1}{T}-0.86276 \times 10^{\circ} \frac{1}{T^{2}} \quad(I I-5)
$$

is the best fit of the points in Figure II-1 to a second order polynomial, when the circled point is excluded, and is plotted as a solid curve. Including the circled point in the regression analysis results in an internal maximum. $A$ linear fit of the points is poor $\left(x^{2}=0.766\right)$, while higher order equations have internal inflection points 


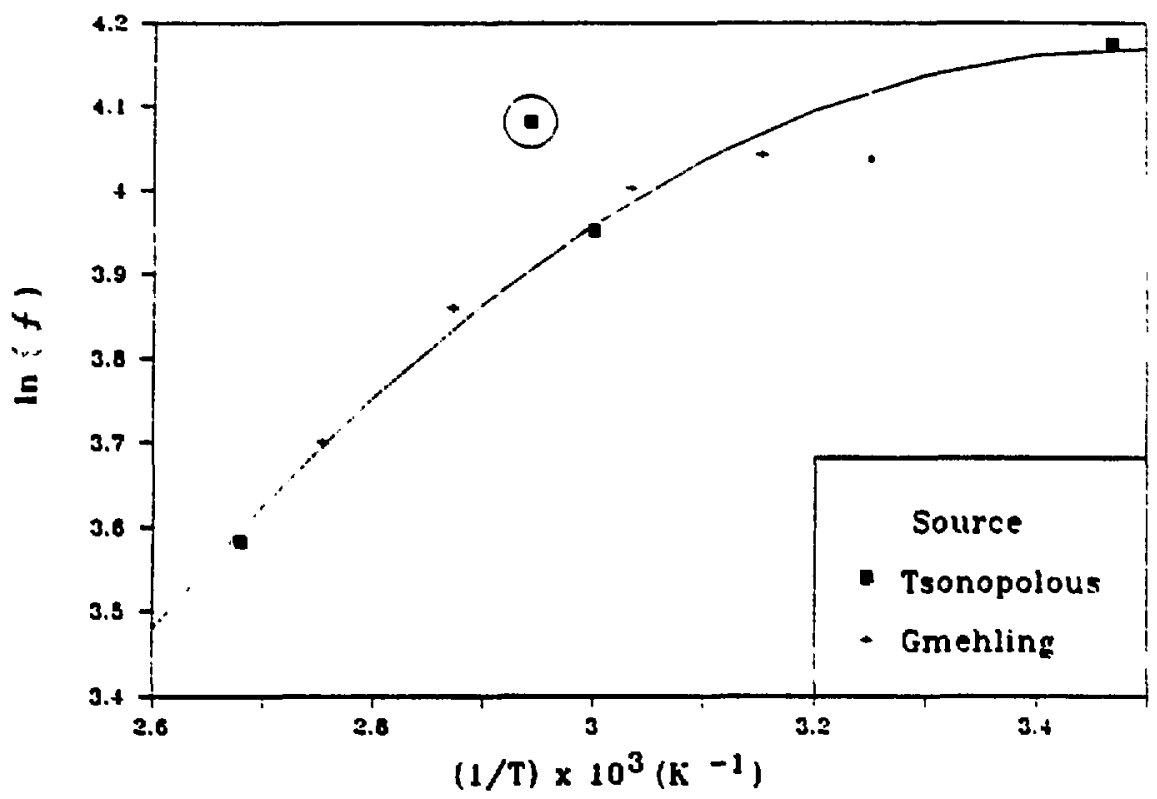

Fizure $11-1$ Activity Coefficients for Phenol in Water at Various Temperatures. The Circled Point was Considered Anomolous. 
and/or extrema. For these reasons Equation II-5 was used in this work to determine activity coefficients for phenol in water at temperatures between 15 and $100^{\circ} \mathrm{C}$.

Equation II-5 is an empirical fit without theoretical basis. The equation is a tool to determine activity coefficients that are reasonable in light of published data. It should be noted, however, that there is some theoretical support for a curve that is concave to the $1 / T$ axis. Tsonopoulos tound this behavior for phenol in water when he analyzed liguid-liquid mutual solubllity data with the van Laar equations and with the wilson equation. The curves lie below the data in Figure II-I, however.

\section{other aromatic Compounde in water}

Tsonopolous (1970) calculated activity coefficients at more than one temperature each for benzene, nitrobenzene, aniline, p-nitrophenol, and p-chlorophenol. Values at $80^{\circ} \mathrm{C}$ were determined using equation II-4. Tsonopolous also calculated the activity coefficients of hydroquinone and resorcinol in aqueous solutions at $25^{\circ} \mathrm{C}$.

Activity coefficients were avallable at $25^{\circ} \mathrm{C}$ for 2,4-dimethylphenol (Banerjee, 1985) and anisole (Tsonopolous, 1970). Values at $80^{\circ} \mathrm{C}$ were calculated from equation II-4 with the parameter B set equal to zero. 
Activity coefficients for p-hydroxybenzaldehyde (phb) and for p-methoxyphenol (pmp) were estimated from solubility data. The solubility of phb at $30.5^{\circ} \mathrm{C}$ is 13.8 $\mathrm{g} / \mathrm{l}$ (Windholz, 1983), and the solubility of pmp at $25^{\circ} \mathrm{C}$ is $40 \mathrm{~g} / \mathrm{l}$ (Verschueren, 1983). Both compounds are solids at these temperatures, so activity coeficients $\left(f_{1}\right)$ are related to mole fraction solubilities $\left(x_{1}\right)$ by:

$$
f_{1}=\frac{1}{X_{1}} \exp \left[\frac{\Delta h_{l}}{R T}\left(1-\frac{T}{T_{m}}\right)\right]
$$

The variable $\left(\Delta h_{1}\right)$ is the heat of fusion of the pure solid at temperature $T, R$ is the gas constant, and $T_{m}$ is the melting point of the solid. Unfortunately, heat of fusion data were not available so, as an approximation, the exponential term was set equal to unity. Using the values thus calculated, activity coefficients at $80^{\circ} \mathrm{C}$ were obtained using equation II-4 with B set equal to zero.

\section{Phenol in Hoptane}

Infinite dilution activity coeficients for phenol in heptane were estimated from solubility data using the UNIQIAC model for liquid phase nonidealities. The governing equation is:

$$
\ln f_{1}^{*}=\ln \frac{r_{1}}{r_{2}}+\frac{z}{2} q_{1} \ln \frac{q_{1} r_{2}}{q_{2} r_{1}}+l_{1}-\frac{r_{1}}{r_{2}} l_{2}-q_{1} \ln T_{21}+q_{1}\left(1-T_{12}\right)(11-7)
$$


Solubility data and corresponding parameters (or means to calculate them) for the UNIQUAC model are reported by Sorensen and Arlt (1979) for the temperatures 35, 40, 45, and $50^{\circ} \mathrm{C}$. Equation II-A was used to obtain values at 25 and $80^{\circ} \mathrm{C}$.

\section{References}

Adamson, A. W. Physical chemintery of surfaces, 4th Ed., John wlley \& Sons, New York, 1982.

Bogart, M.J.P. and Brunjes, A.S. Shem, Eng, Progr, 1948, 44. 95.

Freundlich, H. Solloid and capillary chemistry, Meuthuen, London, 1926.

Gmehling, J, and Onken, U. Yapor-Liouid Equilibria Data collection, volume is part it aoueous-oreanic systems, Behrens, D., and Eckermann, R., Eds., DECHEMA, Frankfurt, Federal Republic of Germany, 1977.

Gmehling, J.: Onken, U.; and Arlt, W. Vapor-Liegrid Equilibria Data cellection. Supolement 1 Aounous-oraanic Systoms, Behrens, D., and Eckermann, R., Eds., DECHEMA, Frankfurt, Federal Republic of Germany, 1981.

Hansen, R.S. and Craig, R.P. I, Physe Chem, 1954, 58, 211. Jaycock, M.J. and Partitt, G.D. Chemistry of Interfaces, John wiley Sons, New York, 1981. 
Kliment, V.; Fried, V.; and Pick, J. collect, Crech, chem. commun,, 1964, 29, 2008.

Markuzin, N.P. I, Appl, Chem, USSR, 1961, 34, 1121.

Reid, R.C.: Prausnitz, J.M.; and Poling, B.E. The

Properties of Gases and Liguids, 4th Ed., McGraw-Hill, New York, 1987.

Prausnitz, J.M. Kolncular Thermedynamies of Eluid-Phase Equilibria, Prentice-Hall, New Jersey, 1969.

Schulek, E.: Pungor, E.: and Trompler J. Kikroehim, Aeta, 1958, 52 .

Sorensen, J.M. and ArIt, w. Liouid-Iiouid Equilibrium Data collection, Binary systems: chomicty pata series vole $v$. Part 1, Behrons, D., and Eckermann, R., Eds., DECHEMA, Frankfurt, Federal Republic of Germany, 1979. Tsonopoulos, C., Ph.D. Dissertatiō̃ tsi Chemical Engineering, University of California, Berkeley, 1970. Verschueren, K. ed. Hanbook of Environmental Data on organic Chemieals, Van Nostrand Reinhold, NY, 1983. Weller, R.; Schuberth, H.: and Leibnitz, E. I. Prakt. cheme, 1963, 21, 234.

Windhol2, M. ed. The kerak Index, loth Ed., Merck Co., Inc., Rahway, N.J., 1983.

Schreinearakers, F.A.H. 2. Phys, chem, 1900, 35, 458 . 